Pure and Applied Mathematics Quarterly

Volume 2, Number 4

(Special Issue: In honor of

Robert MacPherson, Part 2 of 3)

$1255-1312,2006$

\title{
Fusion and Convolution: Applications to Affine Kac-Moody Algebras at the Critical Level
}

\author{
Edward Frenkel and Dennis Gaitsgory \\ Dedicated to Robert MacPherson on his 60th birthday
}

\begin{abstract}
Let $\mathfrak{g}$ be a semi-simple Lie algebra, and let $\widehat{\mathfrak{g}}$ be the corresponding affine Kac-Moody algebra. Consider the category of $\widehat{\mathfrak{g}}$-modules at the critical level, on which the action of the Iwahori subalgebra integrates to an algebraic action of the Iwahori subgroup $I \subset G((t))$. We study the convolution functors on this category which send a $\widehat{\mathfrak{g}}$-module $\mathcal{M}$ to $\mathcal{Z}_{V} \star \mathcal{M}$, where $z_{V}$ is the "central" sheaf on the affine flag scheme $G((t)) / I$, corresponding to a representation $V$ of the Langlands dual group of $G$. We show that each object $\mathcal{M}$ of our category is an "eigen-module" with respect to these functors, that is $z_{V} \star \mathcal{M}$ is isomorphic to $\mathcal{V} \otimes_{\mathfrak{Z}} \mathcal{M}$, where $\mathcal{V}$ is a vector bundle, corresponding to $V$, defined over the spectrum of the center $\mathfrak{Z}$ of our category. In order to establish this isomorphism, we interpret the two sides functorially, using the notion of fusion product of modules over the affine Kac-Moody algebra.
\end{abstract}

\section{INTRODUCTION}

The goal of this article is two-fold. On the one hand, it constitutes the second in the series of papers devoted to the study of the category of representations of an affine Kac-Moody algebra at the critical level in connection with the local geometric Langlands correspondence. On the other hand, we study from the geometric point of view the concept of fusion of modules over an affine KacMoody algebra, or, more generally, chiral modules over a chiral algebra that admits an algebraic group as a group of its symmetries.

Received November 1, 2005. 
0.1. Let us explain the first perspective in some detail. In [FG2] we proposed a framework for the local geometric Langlands correspondence. Namely, let $G$ be a semi-simple algebraic group (over $\mathbb{C}$ or any other algebraically closed field of characteristic 0 ), and let us consider $G((t))$ as a group ind-scheme. We proposed that to a "local Langlands parameter" $\sigma$, which is a $\check{G}$-bundle with a connection on the punctured disc (where $\check{G}$ is the Langlands dual group of $G$ ), there should correspond a category $\mathcal{C}_{\sigma}$ equipped with an action of $G((t))$.

Unfortunately, in general we could not characterize $\mathcal{C}_{\sigma}$ in a unique way by a universal property. However, we conjectured that this category is closely connected to the category of representations of the affine Kac-Moody algebra $\widehat{\mathfrak{g}}$ at the critical level.

To describe this connection, let us recall that, according to $[\mathrm{FF}, \mathrm{F}]$, the center $\mathfrak{Z}_{\mathfrak{g}}$ of the completed enveloping algebra of $\widehat{\mathfrak{g}}_{\text {crit }}$ is isomorphic to the algebra of functions on the space $\mathrm{Op}_{\mathfrak{\mathfrak { g }}}\left(\mathcal{D}^{\times}\right)$of $\check{\mathfrak{g}}$-opers on the punctured disc $\mathcal{D}^{\times}$(where $\check{\mathfrak{g}}$ is the Langlands dual Lie algebra of $\mathfrak{g}$ ). We recall that a $\check{\mathfrak{g}}$-oper on $\mathcal{D}^{\times}$(or, more generally, on any curve) is by definition a $\check{G}$-bundle $\mathcal{F}_{G}$ equipped with a connection $\nabla$, together with an additional datum, namely, a reduction of $\mathcal{F}_{G}$ to a Borel subgroup of $\breve{G}$, which is in a particular relative position with respect to $\nabla$.

For a fixed $\check{\mathfrak{g}}$-oper $\chi$, which we regard as a character of $\mathfrak{Z}_{\mathfrak{g}}$, we can consider the sub-category $\widehat{\mathfrak{g}}_{\text {crit }}-\bmod \chi$ of the category $\widehat{\mathfrak{g}}_{\text {crit-mod }}$ of all discrete modules at the critical level, on which the center acts according to this character. This category carries a canonical action of $G((t))$ via its adjoint action on $\widehat{\mathfrak{g}}_{\text {crit }}$.

We proposed in [FG2] that $\widehat{\mathfrak{g}}_{\text {crit }}-\bmod { }_{\chi}$ should be equivalent to the sought-after category $\mathcal{C}_{\sigma}$, where $\sigma$ corresponds to the pair $\left(\mathcal{F}_{G}, \nabla\right)$ underlying the oper $\chi$. This guess entails a far-reaching corollary that the categories $\widehat{\mathfrak{g}}_{\text {crit }}-\bmod \chi_{\chi^{1}}$ and $\widehat{\mathfrak{g}}_{\text {crit-mod }} \chi_{\chi^{2}}$ for two different central characters $\chi^{1}$ and $\chi^{2}$ are equivalent once we identify the underlying local systems $\left(\mathcal{F}_{G}^{1}, \nabla^{1}\right)$ and $\left(\mathcal{F}_{G}^{2}, \nabla^{2}\right)$.

In [FG2] we discussed various consequences of this proposal, and in particular, a concrete conjecture about the structure of the full subcategory $\widehat{\mathfrak{g}}_{\text {crit }}$-mod nilp of the category of $\widehat{\mathfrak{g}}_{\text {crit }}$-modules. Its objects are $I$-monodromic $\widehat{\mathfrak{g}}_{\text {crit }}$-modules (where $I$ is the Iwahori subgroup of $G((t)))$, which are supported, as $\mathfrak{Z}_{\mathfrak{g}}$-modules, over a certain sub-scheme $\mathrm{Op}_{\mathfrak{\mathfrak { g }}}^{\text {nilp }} \subset \mathrm{Op}_{\mathfrak{\mathfrak { g }}}\left(\mathcal{D}^{\times}\right)$.

Our conjecture was that the derived category of $\widehat{\mathfrak{g}}_{\text {crit }}-\bmod$ nilp $^{I, m}$ is equivalent to the derived category of quasi-coherent sheaves on the scheme $\widetilde{\mathfrak{g}} / \widetilde{G} \times \mathrm{Op}_{\mathfrak{\mathfrak { g }}}^{\text {nilp }}$, where $\check{\mathfrak{g}} / \breve{G}$

$\widetilde{\mathfrak{g}} \rightarrow \check{\mathfrak{g}}$ is Grothendieck's alteration, and $\mathrm{Op}_{\mathfrak{\mathfrak { g }}}^{\text {nilp }} \rightarrow \check{\mathfrak{g}} / \check{G}$ is a canonical residue map 
Res $^{\text {nilp }}$, introduced in [FG2]. In [FG2] we proved a weaker statement that certain quotients of these two categories are equivalent.

In order to prove this conjecture in full we need to know a wide range of results about the structure of the category $\widehat{\mathfrak{g}}_{\text {crit }}$-mod. One class of such results has to do with the Harish-Chandra convolution action of D-modules on $G((t))$ on the derived category $D\left(\widehat{\mathfrak{g}}_{\text {crit }}\right.$-mod $)$.

0.2. In [FG2] we explained, following [BD], that the appropriately defined $I$ equivariant derived category $D^{b}\left(\mathrm{D}\left(\mathrm{Fl}_{G}\right)_{\text {crit }} \text {-mod }\right)^{I}$ of critically twisted D-modules on the affine flag scheme $\mathrm{Fl}_{G}=G((t)) / I$ acts on the $I$-equivariant derived category $D^{b}\left(\widehat{\mathfrak{g}}_{\text {crit-mod }}\right)^{I}$ of $\widehat{\mathfrak{g}}_{\text {crit }}$-modules.

This structure is called the Harish-Chandra convolution action, and its origin is the action of the affine Hecke algebra (whose categorification is $\left.D^{b}\left(\mathrm{D}\left(\mathrm{Fl}_{G}\right)_{\text {crit }}-\bmod \right)^{I}\right)$ on the space of $I$-invariant vectors in any representation of the group $G$ over a local non-archimedian field.

The category $D^{b}\left(\mathrm{D}\left(\mathrm{Fl}_{G}\right)_{\text {crit }} \text {-mod }\right)^{I}$ contains special objects, the so-called central sheaves $z_{V}, V \in \operatorname{Rep}(\check{G})$, introduced in [Ga]. They correspond to the central elements of the affine Hecke algebra.

The main result of the present paper is that all objects of $\widehat{\mathfrak{g}}_{\text {crit }}-\bmod { }^{I}$ are eigenobjects with respect to the functor of convolution with the central sheaves. This is one of the crucial steps in our project describing $\widehat{\mathfrak{g}}_{\text {crit }}$-mod $\operatorname{milp}_{\text {nilp }}^{I, m}$ in terms of quasicoherent sheaves. Let us now explain more precisely what being an eigen-object means in our set-up.

In Sect. 5.2, we explain that the support over the ind-scheme $\operatorname{Spec}\left(\mathfrak{Z}_{\mathfrak{g}}\right)$ of any object from $\widehat{\mathfrak{g}}_{\text {crit }}-\bmod { }^{I}$ is contained in the ind-subscheme $\operatorname{Spec}\left(\widetilde{\mathfrak{Z}}_{\mathfrak{g}}^{\text {int,nilp }}\right)$ that corresponds to opers which, as local systems on $\mathcal{D}^{\times}$, have regular singularities and a unipotent monodromy. We show also that to each $V \in \operatorname{Rep}(\check{G})$ there corresponds a vector bundle $\mathcal{V}_{\widetilde{\mathfrak{Z}}_{\mathfrak{g}} \text { int,nilp }}$ over $\operatorname{Spec}\left(\widetilde{\mathfrak{Z}}_{\mathfrak{g}}^{\text {int,nilp }}\right)$. The geometric meaning of this vector bundle is the following: for a $\mathbb{C}$-point $\chi \in \operatorname{Spec}\left(\widetilde{\mathfrak{Z}}_{\mathfrak{g}}^{\text {int,nilp }}\right) \simeq \mathrm{Op}_{\mathfrak{g}}\left(\mathcal{D}^{\times}\right)$, the fiber $\mathcal{V}_{\chi}$ of $\mathcal{V}_{\widetilde{\mathfrak{Z}}_{\mathfrak{g}} \text { int,nilp is isomorphic to the fiber at the origin of the canonical }}$ (i.e., Deligne's) extension from $\mathcal{D}^{\times}$to $\mathcal{D}$ of the local system $\left(\mathcal{F}_{G}, \nabla\right)$, underlying $\chi$.

The vector bundle $\mathcal{V}_{\widetilde{\mathfrak{Z}}_{\mathfrak{g}}^{\text {int,nilp }}}$ is equipped with a nilpotent endomorphism, corresponding to the monodromy of the underlying oper. 
The main result of the present paper, Theorem 5.4, states that for any $\mathcal{M} \in$ $\widehat{\mathfrak{g}}_{\text {crit-mod }}{ }^{I}$ and $V \in \operatorname{Rep}(\breve{G})$ we have a canonical isomorphism of $\widehat{\mathfrak{g}}_{\text {crit-modules: }}$

$$
z_{V} \star \mathcal{M} \simeq \mathcal{V}_{\widetilde{\mathcal{Z}}_{\mathfrak{g}}^{\text {int,nilp }}} \underset{\widetilde{\mathfrak{Z}}_{\mathfrak{g}}^{\text {int,nilp }}}{\otimes} \mathcal{M},
$$

such that the action of the monodromy on the left hand side, coming from the definition of $z_{V}$ via the nearby cycles functor as in [Ga], goes under this isomorphism to the nilpotent endomorphism of $\mathcal{V}_{\widetilde{\mathfrak{Z}}_{\mathfrak{g}}^{\text {int,nilp }}}$ mentioned above. Moreover, the isomorphisms of (1) for different representations $V$ are compatible, in a natural sense, with the operation of tensor product.

Thus, for example, if $\mathcal{M}$ is an $I$-equivariant $\widehat{\mathfrak{g}}_{\text {crit }}-$ module on which the center $\mathfrak{Z}_{\mathfrak{g}}$ acts via the character corresponding to a particular $\chi \in \widetilde{\mathfrak{Z}}_{\mathfrak{g}}^{\text {int,nilp }}$, then the isomorphism (1) becomes

$$
z_{V} \star \mathcal{M} \simeq \mathcal{V}_{\chi} \underset{\mathbb{C}}{\otimes} \mathcal{M}
$$

where $\mathcal{V}_{\chi}$ is as above.

0.3. The isomorphism stated in (1) has an analogue for the category of $G[[t]]$ equivariant $\widehat{\mathfrak{g}}_{\text {crit }}$-modules (rather than $I$-equivariant ones). In this case the role of the category $\mathrm{D}\left(\mathrm{Fl}_{G}\right)_{\text {crit }}-\bmod ^{I}$ is played by the category $\left.\mathrm{D}\left(\mathrm{Gr}_{G}\right)_{\text {crit }}-\bmod { }^{G[}[t]\right]$ of $G[[t]]$-equivariant D-modules on the affine Grassmannian $\operatorname{Gr}_{G}=G((t)) / G[[t]]$. This category is a tensor category that is equivalent to the category $\operatorname{Rep}(\check{G})$ of finite-dimensional representations of $\check{G}$, see [MV] (it may also be thought of as a categorification of the spherical Hecke algebra).

Hence, for each $V \in \operatorname{Rep}(\check{G})$ we have the corresponding object $\mathcal{F}_{V}$ in the category $\mathrm{D}\left(\mathrm{Gr}_{G}\right)_{\text {crit }}$-mod ${ }^{G[t]]}$. A spherical version of our main theorem, Theorem 1.10 , states that for every $\left.\left.\mathcal{M} \in \widehat{\mathfrak{g}}_{\text {crit }}-\bmod { }^{G[t}\right]\right]$ there is a canonical isomorphism

$$
\mathcal{F}_{V} \star \mathcal{M} \simeq \mathcal{V}_{\widetilde{\mathfrak{J}}_{\mathfrak{g}}^{\text {int,nilp }}} \underset{\widetilde{\mathfrak{J}}_{\mathfrak{g}}^{\text {int,nilp }}}{\otimes} \mathcal{M}
$$

Moreover, we obtain that the support of every such $\mathcal{M}$ over $\operatorname{Spec}\left(\widetilde{\mathfrak{Z}}_{\mathfrak{g}}^{\text {int,nilp }}\right)$ is contained in the ind-subscheme $\operatorname{Spec}\left(\widetilde{\mathfrak{Z}}_{\mathfrak{g}}^{\mathrm{m} . \mathrm{f}}\right)$, corresponding to monodromy-free opers.

Tautologically, the isomorphism (2) is a particular case of that of (1). Let us specialize further to the case when $\mathcal{M} \in \widehat{\mathfrak{g}}_{\text {crit }}$-mod ${ }^{G[t]]}$ is the vacuum module $\mathbb{V}_{\text {crit }}$. In the latter case, the corresponding isomorphism

$$
\mathcal{F}_{V} \star \mathbb{V}_{\text {crit }} \simeq \mathcal{V}_{\mathfrak{z}_{\mathfrak{g}}} \underset{\mathfrak{z g}}{\otimes \mathbb{V}_{\text {crit }}}
$$

is known, due to $[\mathrm{BD}]$. 
The main idea of this paper is that one can derive results such as (1) and (2) from the special case when $\mathcal{M}=\mathbb{V}_{\text {crit }}$. This is based on the operation of fusion product, and this brings us to the discussion of the second perspective in which one can view this paper.

0.4. Let $\mathcal{A}$ be a chiral algebra on a curve $X$, and let $\mathcal{M}_{1}, \mathcal{M}_{2}$ and $\mathcal{N}$ be chiral $\mathcal{A}$ modules. In this case one can consider the set of chiral pairings $\left\{\mathcal{M}_{1}, \mathcal{M}_{2}\right\} \rightarrow \mathcal{N}$. These notions were introduced by A. Beilinson and V. Drinfeld in [CHA].

When $\mathcal{A}$ is a chiral algebra attached to a conformal vertex algebra $V$, and $\mathcal{M}_{1}, \mathcal{M}_{2}, \mathcal{N}$ are obtained from $\mathrm{V}$-modules, the notion of chiral pairing is similar to that of intertwining operator between modules over $\mathrm{V}$, see [FHL].

If for fixed $\mathcal{M}_{1}$ and $\mathcal{M}_{2}$ the functor that assigns to $\mathcal{N}$ the set of chiral pairings $\left\{\mathcal{M}_{1}, \mathcal{M}_{2}\right\} \rightarrow \mathcal{N}$ is representable, we shall call the representing object the fusion product of $\mathcal{M}_{1}$ and $\mathcal{M}_{2}$.

Assume now that $\mathcal{A}$ is the chiral algebra $\mathcal{A}_{\mathfrak{g}, \kappa}$, attached to a semi-simple Lie algebra $\mathfrak{g}$ and a symmetric invariant form $\kappa: \mathfrak{g} \otimes \mathfrak{g} \rightarrow \mathbb{C}$. Then the category of chiral $\mathcal{A}_{\mathfrak{g}, \kappa}$-modules, supported at a given point $x \in X$ is naturally equivalent to the category $\widehat{\mathfrak{g}}_{\kappa}$-mod of representations of the affine Kac-Moody algebra $\widehat{\mathfrak{g}}$ at the level $\kappa$. (More generally, we can consider chiral algebras that admit $G$ as a group of symmetries, see Sect. 4.1).

Let us suppose now that $\kappa$ is non-positive and integral. In this case, to every $V \in \operatorname{Rep}(\check{G})$ we can attach a chiral $\mathcal{A}_{\mathfrak{g}, \kappa}$-module, denoted $\Gamma\left(\operatorname{Gr}_{G, X}, \mathcal{F}_{V, X}\right)$, or $\mathcal{F}_{V, X} \star \mathcal{A}_{\mathfrak{g}, \kappa}$. It is constructed using the D-module $\mathcal{F}_{V} \in \mathrm{D}\left(\operatorname{Gr}_{G}\right)_{\kappa}$-mod ${ }^{G[t]]}$, which is well-defined for every integral $\kappa$.

Let $\mathcal{M}_{2}=\mathcal{M}$ be any $G[[t]]$-equivariant (resp., $I$-equivariant) $\widehat{\mathfrak{g}}_{\kappa}$-module. We set $\mathcal{M}_{1}:=\Gamma\left(\operatorname{Gr}_{G, X}, \mathcal{F}_{V, X}\right)$, or more generally, $\mathcal{M}_{1}:=\Gamma\left(\mathrm{Gr}_{G, X}, \mathcal{F}_{V, X}\right) \otimes \mathcal{E}$, where $\mathcal{E}$ is a local system on the punctured curve $X-x$ with a nilpotent monodromy around $x$.

Our key technical tool is the assertion (see Theorem 2.6 and Theorem 6.6) that in this case fusion products exist and are given by $\mathcal{F}_{V} \star \mathcal{M} \mathbb{C}$ in the $G[[t]]$-equivariant case, and by

$$
\left(\left(z_{V} \star \mathcal{M}\right) \otimes \Psi(\varepsilon)\right)_{N}
$$

in the $I$-equivariant case, where $\Psi(\mathcal{E})$ denotes nearby cycles of $\mathcal{E}$ at $x$, viewed as a vector space with a nilpotent operator, and the subscript $N$ stands for taking coinvariants of the monodromy.

The proofs of the main Theorems, namely, 1.10 and 5.4, are obtained by showing that the right-hand sides of the stated isomorphisms also represent the above 
functors of chiral pairings, and it is this last assertion that uses the result of [BD] about the isomorphism (3).

Let us remark that the proofs of the main theorems do not actually use the full statements of Theorems 2.6 and 6.6, but only the existence of the corresponding maps in one direction, and their compatibility with tensor products of representations.

0.5. Let us briefly describe the way this paper is organized. It is divided into two parts.

In Part I we discuss in detail the $G[[t]]$-equivariant situation. In Sect. 1 we state Theorem 1.10; in Sect. 2 we discuss the relationship between the fusion product and Harish-Chandra convolution and state Theorem 2.6. In Sect. 3 we complete the proof of Theorem 1.10, and finally in Sect. 4 we prove a generalization of Theorem 2.6 in the framework of chiral algebras, endowed with a Harish-Chandra action of the group $G$.

In Part II we show how to modify the material of Part I for the $I$-equivariant situation. Thus, in Sect. 5 we state Theorem 5.4, and in Sect. 6 we derive it from (a part of) Theorem 6.6. In Sect. 7 we prove a suitable generalization of Theorem 6.6.

In the appendix, Sect. 8, we give a proof of the fact that the chiral bracket on chiral algebras such as $\mathcal{A}_{\mathfrak{g}, \kappa}$ can be described using D-modules on the BeilinsonDrinfeld Grassmannian.

The notation in this paper follows closely that of [FG2].

0.6. Acknowledgments. D.G. would like to thank Sasha Beilinson for patient explanations and stimulating discussions.

The research of E.F. was supported by the DARPA grant HR0011-04-1-0031 and by the NSF grant DMS-0303529.

\section{Part I: The spherical case}

\section{Convolution at the CRitical level}

1.1. Recollections. Let $\mathfrak{g}$ be a simple finite-dimensional Lie algebra. For an invariant inner product $\kappa$ on $\mathfrak{g}$ (which is unique up to a scalar) define the central extension $\widehat{\mathfrak{g}}_{\kappa}$ of the formal loop algebra $\mathfrak{g} \otimes \mathbb{C}((t))$ which fits into the short exact sequence

$$
0 \rightarrow \mathbb{C} 1 \rightarrow \widehat{\mathfrak{g}}_{\kappa} \rightarrow \mathfrak{g} \otimes \mathbb{C}((t)) \rightarrow 0 .
$$


This sequence is split as a vector space, and the commutation relations read

$$
[x \otimes f(t), y \otimes g(t)]=[x, y] \otimes f(t) g(t)-\kappa(x, y) \cdot \operatorname{Res}(f \cdot d g) \cdot \mathbf{1},
$$

and $\mathbf{1}$ is a central element. The Lie algebra $\widehat{\mathfrak{g}}_{\kappa}$ is the affine Kac-Moody algebra associated to $\kappa$. We will denote by $\widehat{\mathfrak{g}}_{\kappa}$-mod the category of discrete representations of $\widehat{\mathfrak{g}}_{\kappa}$ (i.e., such that any vector is annihilated by $\mathfrak{g} \otimes t^{n} \mathbb{C}[[t]]$ for sufficiently large $n$ ), on which $\mathbf{1}$ acts as the identity.

Let $\kappa_{K i l}$ be the Killing form:

$$
\kappa_{K i l}(x, y)=\operatorname{Tr}(\operatorname{ad}(x) \circ \operatorname{ad}(y)) .
$$

A level $\kappa$ is called critical (resp., positive, negative, irrational) if $\kappa=c \cdot \kappa_{K i l}$ and $c=-\frac{1}{2}$ (resp., $c+\frac{1}{2} \in \mathbb{Q}^{>0}, c+\frac{1}{2} \in \mathbb{Q}^{<0}, c \notin \mathbb{Q}$ ). A level $\kappa$ is called integral is it is an integral multiple of the standard inner product, normalized so that the square length of the maximal root is equal to 2 .

Next, we recall some notation and terminology from the theory of chiral algebras introduced in [CHA]. Our chiral algebras will be defined on a smooth algebraic curve $X$. We will fix a point $x \in X$, and identify D-modules supported at $x$ with underlying vector spaces.

We will denote by $\mathcal{D}_{x}$ and $\mathcal{D}_{x}^{\times}$, respectively, the formal disc and the formal punctured disc around $x$. If we choose a coordinate $t$ near $x$, we obtain the identifications $\mathcal{D}_{x} \simeq \mathcal{D}:=\operatorname{Spec}(\mathbb{C}[[t]])$ and $\mathcal{D}_{x}^{\times} \simeq \mathcal{D}^{\times}:=\operatorname{Spec}(\mathbb{C}((t)))$,

Following [CHA] (see also [FG2], Sect. 10), we associate to $\mathfrak{g}$ the Lie-* algebra $L_{\mathfrak{g}}=\mathfrak{g} \otimes D_{X}$, and for each level $\kappa$ its central extension $L_{\mathfrak{g}, \kappa}$ by means of $\omega_{X}$. By definition, the chiral algebra $\mathcal{A}_{\mathfrak{g}, \kappa}$ is the quotient of the chiral universal envelope of $L_{\mathfrak{g}, \kappa}$ obtained by identifying the two copies of $\omega_{X}$.

For the rest of this section we shall fix the level $\kappa$ to be critical. Let $\mathfrak{z}_{\mathfrak{g}}$ be the center of $\mathcal{A}_{\mathfrak{g} \text {,crit }}$, viewed as a commutative D-algebra on a curve $X$. Denote by $\mathfrak{z}_{\mathfrak{g}, x}$ the fiber of $\mathfrak{z}_{\mathfrak{g}}$ at the point $x \in X$, and let $\mathfrak{Z}_{\mathfrak{g}}$ be the topological commutative algebra corresponding to $\mathfrak{z}_{\mathfrak{g}}$ and $x$, as defined in [CHA], Sect. 3.6.18.

There exists a canonical map from $\mathfrak{Z}_{\mathfrak{g}}$ to the center of the category $\widehat{\mathfrak{g}}_{\text {crit }}-$ mod . (The latter identifies tautologically with the center of the corresponding completed universal enveloping algebra $\widetilde{U}_{\text {crit }}(\widehat{\mathfrak{g}})$, see [FG2], Sect. 5.1 for more details.) One can show that the map from $\mathfrak{Z}_{\mathfrak{g}}$ to $Z\left(\widetilde{U}_{\text {crit }}(\widehat{\mathfrak{g}})\right)$ is in fact an isomorphism, but we will not use this fact.

Denote by $\check{\mathfrak{g}}$ the Langlands dual Lie algebra to $\mathfrak{g}$. Let $O \mathrm{p}_{\mathfrak{\mathfrak { g }}, X}$ be the D-scheme of $\breve{\mathfrak{g}}$-opers on $X[\mathrm{BD}]$. The following isomorphism is proved in $[\mathrm{FF}, \mathrm{F}]$ :

$$
\mathfrak{z} \mathfrak{g} \simeq \operatorname{Fun}\left(\mathrm{Op}_{\mathfrak{\mathfrak { g }}, X}\right) .
$$


Let consider also the spaces of $\check{\mathfrak{g}}$-opers $\mathrm{Op}_{\check{\mathfrak{g}}}\left(\mathcal{D}_{x}\right)$ and $\mathrm{Op}_{\mathfrak{\mathfrak { g }}}\left(\mathcal{D}_{x}^{\times}\right)$on the formal disc $\mathcal{D}_{x}$ and the formal punctured disc $\mathcal{D}_{x}^{\times}$, respectively. The former is a scheme of infinite type, and the latter is an ind-scheme. We refer the reader to Part I of [FG2] for a detailed discussion of opers.

The isomorphism (5) implies that:

$$
\mathfrak{z}_{\mathfrak{g}, x} \simeq \operatorname{Fun}\left(\mathrm{Op}_{\mathfrak{\mathfrak { g }}}^{\mathrm{reg}}\right),
$$

where $\mathrm{Op}_{\mathfrak{\mathfrak { g }}}^{\mathrm{reg}}:=\mathrm{Op}_{\breve{\mathfrak{g}}}\left(\mathcal{D}_{x}\right)$, and

$$
\mathfrak{Z}_{\mathfrak{g}} \simeq \operatorname{Fun}\left(\mathrm{Op}_{\mathfrak{\mathfrak { g }}}\left(\mathcal{D}_{x}^{\times}\right)\right)
$$

1.2. Vector bundles over opers. Let us denote by $\check{G}$ the simple algebraic group of simply-connected type, corresponding to $\check{\mathfrak{g}}$. Then there is a tautological $\breve{G}$-bundle $\mathcal{P}_{\breve{G}, \mathrm{Op}_{\check{\mathfrak{g}}, X}}$ over $\mathrm{Op}_{\check{\mathfrak{g}}, X}$ equipped with a connection along $X$. For any finite-dimensional representation $V$ of $\check{G}$ we will denote by $\mathcal{V}_{\mathrm{Op}_{\mathfrak{g}, X}}$ the corresponding associated vector bundle over $\mathrm{Op}_{\breve{\mathfrak{g}}, X}$, equipped with a connection along $X$.

Via the isomorphism (5) we can view $\mathcal{V}_{\mathrm{Op}_{\mathfrak{g}, X}}$ as a locally free $\mathfrak{z}_{\mathfrak{g}}$-module with a connection, and we shall also denote it by $\mathcal{V}_{\mathfrak{z} \mathfrak{g}}$. (We remark that as a $\mathfrak{z}_{\mathfrak{g}}$-module, $\mathcal{V}_{\mathfrak{z}_{\mathfrak{g}}}$ is actually free, but there is no canonical choice of generators.) The fiber of $\mathcal{V}_{\mathfrak{z} \mathfrak{g}}$ over $x \in X$ is a locally free $\mathfrak{z}_{\mathfrak{g}, x}$-module, which we will denote by $\mathcal{V}_{\mathfrak{z} \mathfrak{g}, x}$.

Denote by $\widetilde{\mathfrak{z}}_{\mathfrak{g}, x}$ the topological algebra equal to the completion of $\mathfrak{Z}_{\mathfrak{g}}$ with respect to the ideal defining $\mathfrak{z}_{\mathfrak{g}, x}$, i.e.,

$$
\widetilde{\mathfrak{z}} \mathfrak{g}, x:=\longleftarrow_{n}^{\lim } \mathfrak{\mathfrak { Z } _ { \mathfrak { g } }} / \mathcal{\partial}_{n},
$$

where $\mathcal{J}_{n}$ is the closure of the $n$th power of $\operatorname{ker}\left(\mathfrak{Z}_{\mathfrak{g}} \rightarrow \mathfrak{z}_{\mathfrak{g}, x}\right)$. Let $\operatorname{Spec}\left(\widetilde{\mathfrak{z}}_{\mathfrak{g}, x}\right)$ be the resulting ind-subscheme of $\operatorname{Spec}\left(\mathfrak{Z}_{\mathfrak{g}}\right)$.

(In what follows, for a topological algebra $A \simeq \lim A_{i}$, we shall denote by $\operatorname{Spec}(A)$ the corresponding ind-scheme, i.e., " lim" $\operatorname{Spec}\left(A_{i}\right)$. By a vector bundle over an ind-scheme we shall mean a compatible system of vector bundles over its closed subschemes.)

\section{Proposition-Construction 1.3.}

(1) The vector bundle $\mathcal{V}_{\mathfrak{z}_{\mathfrak{g}, x}}$ naturally extends to a vector bundle $\mathcal{V}_{\tilde{\mathfrak{z}}_{\mathfrak{g}}, x}$ over $\operatorname{Spec}\left(\widetilde{\mathfrak{z}}_{\mathfrak{g}, x}\right)$.

(2) For $V, W \in \operatorname{Rep}(\check{G})$ and $U=V \otimes W$, there is a natural isomorphism $\mathcal{V}_{\widetilde{\mathfrak{z}}_{\mathfrak{g}, x}} \otimes$ $\mathcal{W}_{\tilde{\mathfrak{z}} \mathfrak{g}, x} \simeq \mathcal{U}_{\tilde{\mathfrak{z}}_{\mathfrak{g}, x}}$. 
(3) Each $\mathcal{V}_{\widetilde{\mathfrak{z}} \mathfrak{g}, x}$ is equipped with a (pro)-nilpotent endomorphism $N_{\mathcal{V}_{\tilde{\mathfrak{s}} \mathfrak{g}, x}}$, and these endomorphisms are compatible with the identifications of (2) above.

The rest of this subsection is devoted to the proof of this proposition.

By definition,

$$
\widetilde{\mathfrak{z}} \mathfrak{g}, x \simeq \underbrace{\lim _{\mathfrak{g}}}_{\mathfrak{z}_{\mathfrak{g}}^{\prime}} \mathfrak{z}_{\mathfrak{g}, x}^{\prime},
$$

where $\mathfrak{z}_{\mathfrak{g}}^{\prime}$ runs over the family of D-subalgebras of $\mathfrak{z}_{\mathfrak{g}}$, such that the ideal $\operatorname{ker}\left(\mathfrak{z}_{\mathfrak{g}, x}^{\prime} \rightarrow\right.$ $\mathfrak{z}_{\mathfrak{g}, x}$ ) is nilpotent (here $\mathfrak{z}_{\mathfrak{g}, x}^{\prime}$ denotes the fiber of $\mathfrak{z}_{\mathfrak{g}}^{\prime}$ at $x$ ). Consider the following general set-up:

Let $\mathcal{B}$ be a commutative chiral algebra on $X$, and let $\mathcal{V}_{X-x}$ be a free finite-rank $\mathcal{B}$-module, equipped with a compatible connection along $X$. Assume that $\mathcal{V}_{X-x}$ admits an extension to a free finite rank $\mathcal{B}$-module $\mathcal{V}_{X}$, on which the connection along $X$ has a pole of order $\leq 1$ at $x$ and its residue $\operatorname{Res}\left(\nabla, \mathcal{V}_{X}\right)$, thought of as an endomorphism of the fiber $\mathcal{V}_{x}$ of $\mathcal{V}_{X}$ at $x$, is nilpotent.

Let $\mathcal{B}^{\prime} \hookrightarrow \mathcal{B}$ be a chiral subalgebra, such that $\left.\left.\mathcal{B}^{\prime}\right|_{X-x} \simeq \mathcal{B}\right|_{X-x}$, and such that $\operatorname{ker}\left(\mathcal{B}_{x}^{\prime} \rightarrow \mathcal{B}_{x}\right)$ is nilpotent.

Lemma 1.4. Under the above circumstances, $\mathcal{V}_{X-x}$, viewed as a $\mathcal{B}^{\prime}$-module over $X-x$, admits a unique extension to a free finite rank $\mathcal{B}^{\prime}$-module $\mathcal{V}_{X}^{\prime}$ such that $\mathcal{V}_{X} \simeq \mathcal{V}_{X}^{\prime} \underset{\mathcal{B}^{\prime}}{\otimes} \mathcal{B}$, and such that the connection on $\mathcal{V}_{X}^{\prime}$ has a pole of order $\leq 1$ at $x$. In this case $\operatorname{Res}\left(\nabla, \mathcal{V}_{X}^{\prime}\right)$ is also nilpotent.

Applying this lemma in our situation we obtain a locally free sheaf $\mathcal{V}_{\mathfrak{z}_{\mathfrak{g}}^{\prime}}$ over each $\mathfrak{z}_{\mathfrak{g}}^{\prime}$ as above. Moreover, the formation of $\mathcal{V}_{\mathfrak{z}_{\mathfrak{g}}^{\prime}}$ is compatible with tensor products of representations, by the uniqueness statement of the lemma.

By taking the fiber of $\mathcal{V}_{\mathfrak{z}_{\mathfrak{g}}^{\prime}}$ at $x$ we obtain a vector bundle $\mathcal{V}_{\mathfrak{z}_{\mathfrak{g}}^{\prime}, x}$ over each $\operatorname{Spec}\left(\mathfrak{z}_{\mathfrak{g}, x}^{\prime}\right)$ as above, i.e., a vector bundle over $\operatorname{Spec}\left(\widetilde{\mathfrak{z}}_{\mathfrak{g}, x}\right)$. The endomorphisms $\left.N_{\mathcal{V}_{\tilde{\mathfrak{z}} \mathfrak{g}, x}}\right|_{\operatorname{Spec}\left(\mathfrak{z}_{\mathfrak{g}, x}^{\prime}\right)}$ are equal to $\operatorname{Res}\left(\nabla, \mathcal{V}_{\mathfrak{z}_{\mathfrak{g}}^{\prime}}\right)$.

Let us now prove Lemma 1.4. We have the natural morphisms $\mathcal{B} \rightarrow \mathcal{B}_{x}[[t]]$ and $\mathcal{B}^{\prime} \rightarrow \mathcal{B}_{x}^{\prime}[[t]]$, compatible with connections. It follows from the BeauvilleLaszlo theorem [BL] (see also [BD]) that the problem of extension of $\mathcal{V}_{X-x}$ to $X$ translates to a problem of extensions of locally free modules with connections over $\mathcal{B}_{x}[[t]]$ and $\mathcal{B}_{x}^{\prime}[[t]]$. Thus, we have to prove the following:

Lemma 1.5. Let $B^{\prime} \rightarrow B$ be a surjection of commutative algebras with a nilpotent kernel. Let $T^{\prime}$ be a free module over $B^{\prime}((t))$, endowed with a connection along $t$, and let $T$ be the corresponding $B((t))$-module. Let $T_{0} \subset T$ be a $B[[t]]$-lattice, such that $t \partial_{t} \cdot T_{0} \subset T_{0}$, and the endomorphism induced on $T_{0} / t \cdot T_{0}$ is nilpotent. Then 
there exists a unique $B^{\prime}[[t]]$-lattice $T_{0}^{\prime}$ in $T^{\prime}$ with the same properties, such that $T_{0}^{\prime} \underset{B^{\prime}[[t]]}{\otimes} B[[t]]=T_{0} \subset T$.

Proof. By induction, we can assume that $I:=\operatorname{ker}\left(B^{\prime} \rightarrow B\right)$ is such that $I^{2}=0$. Then we have a short exact sequence

$$
0 \rightarrow I \cdot T^{\prime} \rightarrow T^{\prime} \rightarrow T^{\prime} / I \cdot T^{\prime} \rightarrow 0,
$$

where both $I \cdot T^{\prime} \simeq I \underset{B}{\otimes} T$ and $T^{\prime} / I \cdot T^{\prime} \simeq T$ are $B((t))$-modules.

It is easy to see that $T^{\prime}$ admits at least one $B^{\prime}[[t]]$-lattice ${ }^{\prime} T_{0}^{\prime}$, satisfying

$$
{ }^{\prime} T_{0}^{\prime} \bmod I \cdot T^{\prime}=T_{0} \text { and }{ }^{\prime} T_{0}^{\prime} \cap I \cdot T^{\prime}=I[[t]] \underset{B[[t]]}{\otimes} T_{0} .
$$

The operator $t \partial_{t}$, acting on ' $T_{0}^{\prime}$ defines a $B[[t]]$-linear map

$$
\phi: T_{0} \rightarrow I[[t]] \underset{B[[t]]}{\otimes} T / T_{0} .
$$

Any other lattice $T_{0}^{\prime}$ in $T^{\prime}$ that satisfies (8) differs from ' $T_{0}^{\prime}$ by a $B[[t]]$-linear operator

$$
E: T_{0} \rightarrow I[[t]] \underset{B[[t]]}{\otimes} T / T_{0} .
$$

The condition for $T_{0}^{\prime}$ to satisfy the assumption of the lemma reads as follows:

$$
\phi=\left[E, t \partial_{t}\right] .
$$

This equation is uniquely solvable by induction on the order of the pole, since $\phi-k \cdot$ Id is invertible whenever $k \neq 0$.

1.6. A generalization. Let $V^{\mu}$ be the irreducible $\mathfrak{g}$-module with highest weight $\mu$, and set $\mathbb{V}^{\mu}=\operatorname{Ind}_{\mathfrak{g}[[t]]}^{\widehat{\mathfrak{g}}_{\text {crit }}}\left(V^{\mu}\right)$. Recall from [FG2], Sect. 7.6 that to any dominant integral weight $\lambda$ we have attached a subscheme $\operatorname{Spec}\left(\mathfrak{Z}_{\mathfrak{g}}^{\lambda \text {,reg }}\right) \subset \operatorname{Spec}\left(\mathfrak{Z}_{\mathfrak{g}}\right)$. The following will be proved in Sect. 5.5:

Lemma 1.7. The support of $\mathbb{V}^{\mu}$ over $\operatorname{Spec}\left(\mathfrak{Z}_{\mathfrak{g}}\right)$ is contained in $\operatorname{Spec}\left(\mathfrak{Z}_{\mathfrak{g}}^{\mu, \text { reg }}\right)$.

Let $\mathfrak{z}_{\mathfrak{g}}^{\lambda \text {,reg }}$ be the commutative chiral algebra on $X$, isomorphic to $\mathfrak{z}_{\mathfrak{g}}$ over $X-x$ whose fiber at $x$ is $\operatorname{Spec}\left(\mathfrak{Z}_{\mathfrak{g}}^{\lambda, \text { reg }}\right)$. According to [FG2], Sect. 2.9, for $V \in \operatorname{Rep}(\check{G})$, the locally free sheaf $\mathcal{V}_{\mathfrak{z} \mathfrak{g}}$ with a connection, defined on $\left.\mathfrak{z}_{\mathfrak{g}}^{\lambda \text {,reg }}\right|_{X-x}$, extends to a locally free sheaf with a regular connection over $\mathfrak{z}_{\mathfrak{g}}^{\lambda, \text { reg }}$. We will denote the resulting vector bundle over $\operatorname{Spec}\left(\mathfrak{Z}_{\mathfrak{g}}^{\lambda, \text { reg }}\right)$ by $\mathcal{V}_{\mathfrak{Z}_{\mathfrak{g}}^{\lambda, \text { reg }}}$. 
Let $\widetilde{\mathfrak{Z}}_{\mathfrak{g}}^{\lambda \text {,reg }}$ be the formal completion of $\operatorname{Spec}\left(\mathfrak{Z}_{\mathfrak{g}}\right)$ along $\operatorname{Spec}\left(\mathfrak{Z}_{\mathfrak{g}}^{\lambda, \text { reg }}\right)$. The proof of Proposition-Construction 1.3 implies that $\mathcal{V}_{\mathfrak{Z}_{\mathfrak{g}}^{\lambda, \text { reg }}}$ naturally extends to a vector bundle $\mathcal{V}_{\widetilde{\mathfrak{Z}}_{\mathfrak{g}}^{\lambda, \text { reg }}}$ over $\operatorname{Spec}\left(\widetilde{\mathfrak{Z}}_{\mathfrak{g}}^{\lambda \text {,reg }}\right)$, equipped with a nilpotent endomorphism $N_{\mathcal{V}_{\tilde{\mathfrak{z}} \boldsymbol{\mathfrak { g }}} \text {,reg }}$, in a way compatible with tensor products of objects of $\check{G}$.

Let us denote by $\operatorname{Spec}\left(\mathfrak{Z}_{\mathfrak{g}}^{\text {int,reg }}\right)$ (resp., $\left.\operatorname{Spec}\left(\widetilde{\mathfrak{Z}}_{\mathfrak{g}}^{\text {int,reg }}\right)\right)$ the ind-subscheme of

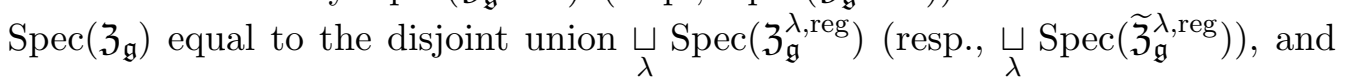
by $\mathcal{V}_{\mathfrak{Z}_{\mathfrak{g}}^{\text {int,reg }}}\left(\right.$ resp., $\left.\mathcal{V}_{\widetilde{\mathfrak{Z}}_{\mathfrak{g}} \text { int,reg }}\right)$ the vector bundle on it, corresponding to $V \in \operatorname{Rep}(\check{G})$. We shall denote by $N_{\mathcal{\mathfrak { \mathcal { F } }}_{\mathfrak{g}} \text { int,reg }}$ the endomorphism of the latter vector bundle.

Let $\operatorname{Spec}\left(\mathfrak{Z}_{\mathfrak{g}}^{\text {m.f. }}\right) \subset \operatorname{Spec}\left(\mathfrak{Z}_{\mathfrak{g}}\right)$ be the sub-functor that corresponds to opers that are monodromy-free as local systems. It is easy to see that we have the following inclusions of functors:

$$
\operatorname{Spec}\left(\mathfrak{Z}_{\mathfrak{g}}^{\text {int,reg }}\right) \subset \operatorname{Spec}\left(\mathfrak{Z}_{\mathfrak{g}}^{\text {m.f. }}\right) \subset \operatorname{Spec}\left(\widetilde{\mathfrak{Z}}_{\mathfrak{g}}^{\text {int,reg }}\right) .
$$

(In fact, one can show that $\operatorname{Spec}\left(\mathfrak{Z}_{\mathfrak{g}}^{\text {m.f. }}\right)$ is the minimal ind-subscheme of $\operatorname{Spec}\left(\widetilde{\mathfrak{Z}}_{\mathfrak{g}}^{\text {int,reg }}\right)$, containing $\operatorname{Spec}\left(\mathfrak{Z}_{\mathfrak{g}}^{\text {int,reg }}\right)$, stable under the action of the Lie algebroid $\Omega^{1}\left(\mathfrak{Z}_{\mathfrak{g}}\right)$, see [FG1], Sect. 6.12.)

Moreover, from the definitions it follows that $\operatorname{Spec}\left(\mathfrak{Z}_{\mathfrak{g}}^{\text {m.f. }}\right)$ is the closed indsubscheme of $\operatorname{Spec}\left(\widetilde{\mathfrak{Z}}_{\mathfrak{g}}^{\text {int,reg }}\right)$, equal to the locus of vanishing of $N_{\mathcal{V}_{\widetilde{\mathfrak{Z}}_{\mathfrak{g}} \text {,reg }}}$ for all $V \in \check{G}$.

Let $\mathcal{M}$ be an object of $\mathfrak{Z}_{\mathfrak{g}}$-mod, i.e., a (discrete) vector space, endowed with a continuous action of $\mathfrak{Z}_{\mathfrak{g}}$. We shall say that $\mathcal{M}$ is supported on $\operatorname{Spec}\left(\widetilde{\mathfrak{Z}}_{\mathfrak{g}}^{\lambda \text {,reg }}\right)$ if every element of $\mathcal{M}$ is annihilated by some power of the ideal of $\mathfrak{Z}_{\mathfrak{g}}^{\lambda \text {,reg }}$ in $\mathfrak{Z}_{\mathfrak{g}}$.

We shall say that a module $\mathcal{M}$ is supported on $\operatorname{Spec}\left(\widetilde{\mathfrak{Z}}_{\mathfrak{g}}^{\text {int,reg }}\right)$ if it is a union (or, automatically, a direct sum) of module $\mathcal{M}^{\lambda}$, where each $\mathcal{M}^{\lambda}$ is supported on $\operatorname{Spec}\left(\widetilde{\mathfrak{Z}}_{\mathfrak{g}}^{\lambda, \text { reg }}\right){ }^{1}$

Given $V \in \operatorname{Rep}(\check{G})$ we have a well-defined functor on the category of $\mathfrak{Z}_{\mathfrak{g}^{-}}$ modules, supported on $\operatorname{Spec}\left(\widetilde{\mathfrak{Z}}_{\mathfrak{g}}^{\text {int,reg }}\right)$ :

$$
\mathcal{M} \mapsto \nu_{\widetilde{\mathfrak{Z}}_{\mathfrak{g}}^{\text {int,reg }}} \underset{\widetilde{\mathfrak{Z}}_{\mathfrak{g}}^{\text {int,reg }}}{\otimes} \mathcal{M} .
$$

Moreover, these functors come equipped with nilpotent endomorphisms $N_{\mathcal{V}_{\widetilde{\mathfrak{z}} \mathfrak{g}} \text {, reg }}$, compatible with tensor products of objects of $\operatorname{Rep}(\check{G})$.

\footnotetext{
${ }^{1}$ In fact, this notion makes sense for any pair of affine ind-schemes, one being a closed indsubscheme of the other.
} 
The above constructions have a relevance for us due to the following:

Lemma 1.8. If $\mathcal{M}$ is a $G[[t]]$-integrable $\widehat{\mathfrak{g}}_{\mathrm{crit}}$-module, then, viewed as a module over $\mathfrak{Z}_{\mathfrak{g}}$, it is supported on $\operatorname{Spec}\left(\widetilde{\mathfrak{Z}}_{\mathfrak{g}}^{\text {int,reg }}\right)$.

The proof follows from Lemma 1.7, since every object of $\widehat{\mathfrak{g}}_{\text {crit }}$-mod ${ }^{G[t]]}$ admits a filtration, such that each subquotient is isomorphic to a quotient module of some $\mathbb{V}^{\lambda}$. (For a different argument see Sect. 5.5.)

1.9. Statement of the main theorem (spherical case). Let $G$ be the group of adjoint type with the Lie algebra $\mathfrak{g}$, so that the Langlands dual group $\check{G}$ is simply-connected. We denote by $\mathrm{Gr}_{G}=G((t)) / G[[t]]$ the corresponding affine Grassmannian. $^{2}$

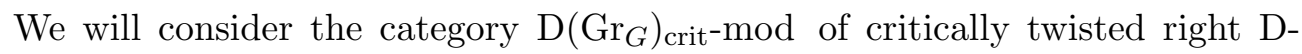
modules on $\mathrm{Gr}_{G}$. Since the critical level is integral, this category is equivalent to category of usual right D-modules on $\mathrm{Gr}_{G}$, via the tensor product by the corresponding line bundle. Let $\mathrm{D}\left(\mathrm{Gr}_{G}\right)_{\text {crit }}-\bmod { }^{G[t]]}$ be the corresponding category of $G[[t]]$-equivariant twisted D-modules.

The geometric Satake equivalence (see $[\mathrm{MV}]$ ) defines a functor

$$
V \in \operatorname{Rep}(\check{G}) \mapsto \mathcal{F}_{V} \in \mathrm{D}\left(\operatorname{Gr}_{G}\right)_{\text {crit }} \text { mod } G[[t]] .
$$

Moreover, $\mathrm{D}\left(\mathrm{Gr}_{G}\right)_{\text {crit }}$-mod ${ }^{G[t]]}$ is endowed with a structure of tensor category via the convolution product, such that the above functor becomes an equivalence of tensor categories.

To $\mathcal{M} \in \widehat{\mathfrak{g}}_{\text {crit-mod }}{ }^{G[t]]}$ and $\mathcal{F} \in \mathrm{D}\left(\mathrm{Gr}_{G}\right)_{\text {crit- }}$-mod we can associate their convolution

$$
\mathcal{F} \star \mathcal{M} \in D\left(\widehat{\mathfrak{g}}_{\text {crit }}\right),
$$

(see [FG2], Sect. 22.5 for the corresponding definitions).

If $\mathcal{F}$ is an object of $\mathrm{D}\left(\mathrm{Gr}_{G}\right)_{\text {crit }}$-mod ${ }^{G[[t]]}$, then $\mathcal{F} \star \mathcal{M}$ will be naturally an object of $D\left(\widehat{\mathfrak{g}}_{\text {crit }}\right)^{G[[t]]}$.

Theorem 1.10. For $\mathcal{M} \in \widehat{\mathfrak{g}}_{\text {crit }}-\bmod { }^{G[[t]]}$ and $V \in \operatorname{Rep}(\check{G})$, the convolution $\mathcal{F}_{V} \star \mathcal{M}$ is acyclic off cohomological degree 0 , and we have a functorial isomorphism

$$
\mathfrak{s}_{V}: \mathcal{F}_{V} \star \mathcal{M} \simeq \mathcal{V}_{\widetilde{\mathfrak{Z}}_{\mathfrak{g}}^{\text {int,reg }}} \underset{\widetilde{\mathfrak{Z}}_{\mathfrak{g}}^{\text {int,reg }}}{\otimes} \mathcal{M},
$$

\footnotetext{
$G$

${ }^{2}$ Note that $\mathrm{Gr}_{G}$ has connected components labeled by elements of the fundamental group of
} 
compatible with tensor products of $\breve{G}$-representations, i.e., for $V, W \in \check{G}$ the diagrams

$$
\begin{gathered}
\mathcal{F}_{V} \star\left(\mathcal{F}_{W} \star \mathcal{M}\right) \stackrel{\mathfrak{s}_{V}}{\longrightarrow} \quad \mathcal{V}_{\widetilde{\mathfrak{Z}}_{\mathfrak{g}}^{\text {int,reg }}} \underset{\widetilde{\mathfrak{Z}}_{\mathfrak{g}}^{\text {int,reg }}}{\otimes}\left(\mathcal{F}_{W} \star \mathcal{M}\right) \\
\sim \downarrow \\
\left(\mathcal{F}_{V} \star \mathcal{F}_{W}\right) \star \mathcal{M} \stackrel{\operatorname{id}_{V} \otimes \mathfrak{s}_{W} \downarrow}{\stackrel{\mathfrak{s}_{V} \otimes W}{\longrightarrow}} \mathcal{V}_{\widetilde{\mathfrak{Z}}_{\mathfrak{g}}^{\text {int,reg }}} \underset{\widetilde{\mathfrak{Z}}_{\mathfrak{g}}^{\text {int,reg }}}{\otimes} \mathcal{W}_{\widetilde{\mathfrak{Z}}_{\mathfrak{g}}^{\text {int,reg }}} \underset{\widetilde{\mathfrak{J}}_{\mathfrak{g}}^{\text {int,reg }}}{\otimes} \mathcal{M},
\end{gathered}
$$

and

$$
\begin{aligned}
& \mathcal{F}_{V} \star\left(\mathcal{F}_{W} \star \mathcal{M}\right) \stackrel{\operatorname{id}_{V} \star \mathfrak{s}_{W}}{\longrightarrow} \quad \mathcal{F}_{V} \star\left(\mathcal{W}_{\widetilde{\mathfrak{Z}}_{\mathfrak{g}}^{\text {int,reg }}} \underset{\widetilde{\mathfrak{Z}}_{\mathfrak{g}}^{\text {int,reg }}}{\otimes} \mathcal{M}\right) \\
& \sim \mathfrak{s}_{V} \downarrow \\
& \left(\mathcal{F}_{V} \star \mathcal{F}_{W}\right) \star \mathcal{M} \quad \stackrel{\mathfrak{s}_{V \otimes W}}{\longrightarrow} \mathcal{V}_{\widetilde{\mathfrak{Z}}_{\mathfrak{g}}^{\text {int,reg }}} \underset{\widetilde{\mathcal{J}}_{\mathfrak{g}}^{\text {int,reg }}}{\otimes} \mathcal{W}_{\widetilde{\mathfrak{J}}_{\mathfrak{g}}^{\text {int,reg }}} \underset{\widetilde{\mathcal{J}}_{\mathfrak{g}}^{\text {int,reg }}}{\otimes} \mathcal{M},
\end{aligned}
$$

are commutative. The endomorphism on $R H S$ of $(9)$, given by $N_{\mathcal{V}_{\widetilde{\mathfrak{z}}} \text { int,reg }}$, is identically equal to 0.

The last statement of Theorem 1.10 implies the following statement, conjectured by A. Beilinson (see [FG1], Conjecture 6.13):

Corollary 1.11. The support in $\operatorname{Spec}\left(\mathfrak{Z}_{\mathfrak{g}}\right)$ of any $G[[t]]$-integrable $\widehat{\mathfrak{g}}_{\text {crit }}$-module is contained in $\operatorname{Spec}\left(\mathfrak{Z}_{\mathfrak{g}}^{\text {m.f. }}\right)$.

1.12. The case of differential operators on the group. Let $\mathfrak{D}_{G \text {,crit }}$ denote the chiral algebra of differential operators on $G$ at the critical level introduced in [AG]. It comes equipped homomorphisms of chiral algebras

$$
\mathfrak{l}: \mathcal{A}_{\mathfrak{g}, \text { crit }} \rightarrow \mathfrak{D}_{G, \text { crit }} \rightarrow \mathcal{A}_{\mathfrak{g}, \text { crit }}: \mathfrak{r}
$$

whose images mutually Lie-* commute. Let $\mathfrak{D}_{G, \text { crit, } x}$ be its fiber at $x$, which we view as a $G[[t]]$-equivariant bimodule at the critical level.

Using the map $\pi: G((t)) \rightarrow \mathrm{Gr}_{G}$, starting from $\mathcal{F} \in \mathrm{D}\left(\mathrm{Gr}_{G}\right)_{\kappa}$-mod (for any level $\kappa$ ), we produce a chiral $\mathfrak{D}_{G, \text { crit-module supported at } x \text { by considering }}$

$$
\Gamma\left(G((t)), \pi^{*}(\mathcal{F})\right) .
$$

We can also view it as $\widehat{\mathfrak{g}}_{\text {crit }}$-bimodule, which is $G[[t]]$-equivariant with respect to the $\mathfrak{r}$ action.

We have:

$$
\Gamma\left(G((t)), \pi^{*}(\mathcal{F})\right) \simeq \mathcal{F} \star \mathfrak{D}_{G, \text { crit }, x},
$$




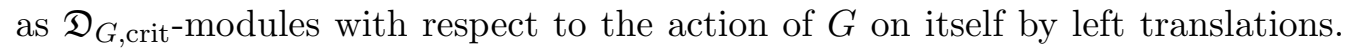
Hence, the corresponding isomorphism holds also on the level of $\widehat{\mathfrak{g}}_{\text {crit }}$-bimodules. Theorem 1.10 then immediately implies the following:

Theorem 1.13. We have a canonical isomorphism of $\widehat{\mathfrak{g}}_{\text {crit-bimodules, }}$

$$
\Gamma\left(G((t)), \pi^{*}\left(\mathcal{F}_{V}\right)\right) \simeq \mathcal{V}_{\widetilde{\mathfrak{Z}}_{\mathfrak{g}}^{\text {int,reg }}} \underset{\widetilde{\mathfrak{Z}}_{\mathfrak{g}}^{\text {int,reg }}}{\otimes} \mathfrak{D}_{G, \text { crit }, x},
$$

where the tensor product is taken with respect to the $\mathfrak{Z}_{\mathfrak{g}}$-module structure on $\mathfrak{D}_{G, \text { crit, } x}$, given by $\mathfrak{l}$. These isomorphisms are compatible with tensor products of objects of $\operatorname{Rep}(\breve{G})$. The endomorphism on the RHS of $(13)$, given by $N_{\mathcal{V}_{\widetilde{\mathfrak{z}}}^{\text {int,reg }}}$, is zero.

Conversely, Theorem 1.13 implies the first two statements of Theorem 1.10:

Proof. Recall (see [FG2], Sect. 21.13) that for a $G[[t]]$-integrable $\widehat{\mathfrak{g}}_{\kappa}$-module $\mathcal{M}$ (at any level $\kappa$ ) and $\mathcal{F} \in \mathrm{D}\left(\mathrm{Gr}_{G}\right)_{\kappa}$-mod there exists a canonical isomorphism of individual cohomologies

$$
h^{i}(\mathcal{F} \star \mathcal{M}) \simeq h^{i}\left(\Gamma\left(G((t)), \pi^{*}(\mathcal{F})\right) \underset{\mathfrak{g}((t)), \mathfrak{g}}{\stackrel{\frac{\infty}{2}}{\otimes}} \mathcal{M}\right),
$$

where the semi-infinite Tor is taken with respect to action of $\widehat{\mathfrak{g}}_{\kappa^{\prime}}$ on $\Gamma(G((t)), \mathcal{F})$ given by $\mathfrak{r}$ (here $\kappa^{\prime}$ is the opposite level). We refer the reader to [FG2] for the precise definition of this functor.

Applying this to $\mathcal{F}=\mathcal{F}_{V}$, and using the isomorphism of Theorem 1.13, we obtain that the cohomologies of $\mathcal{F}_{V} \star \mathcal{M}$ are isomorphic to those of

$$
\left(\mathcal{V}_{\widetilde{\mathcal{Z}}_{\mathfrak{g}}^{\text {int,reg }}} \underset{\widetilde{\mathfrak{Z}}_{\mathfrak{g}}^{\text {int,reg }}}{\otimes} \mathfrak{D}_{G, \text { crit }, x}\right) \underset{\mathfrak{g}((t)), \mathfrak{g}}{\stackrel{\infty}{2}} \mathcal{M} \text {. }
$$

However, since $\mathcal{V}_{\widetilde{\mathfrak{Z}}_{\mathfrak{g}}^{\text {int,reg }}}$ is locally free over $\widetilde{\mathfrak{Z}}_{\mathfrak{g}}^{\text {int,reg }}$, we obtain that the complex (15) is quasi-isomorphic to

$$
\mathcal{V}_{\widetilde{\mathfrak{Z}}_{\mathfrak{g}}^{\text {int,reg }}} \underset{\widetilde{\mathfrak{J}}_{\mathfrak{g}}^{\text {int,reg }}}{\otimes}\left(\mathfrak{D}_{G, \text { crit }, x} \underset{\mathfrak{g}((t)), \mathfrak{g}}{\stackrel{\frac{\infty}{2}}{\otimes}} \mathcal{M}\right) .
$$

However, by [FG2], Corollary 21.14, at any level $\kappa$, we have a quasiisomorphism of $\widehat{\mathfrak{g}}_{\kappa}$-modules

$$
\mathfrak{D}_{G, \kappa, x} \underset{\mathfrak{g}((t)), \mathfrak{g}}{\stackrel{\infty}{2}} \mathcal{M} \simeq \mathcal{M}
$$


Combining (16) and (17) we obtain that $\mathcal{F} \star \mathcal{M}$ has the same cohomologies as $\mathcal{V}_{\widetilde{\mathcal{Z}}_{\mathfrak{g}}^{\text {int,reg }}} \underset{\widetilde{\mathfrak{Z}}_{\mathfrak{g}}^{\text {int,reg }}}{\otimes} \mathcal{M}$, as contended.

\section{INTERPRETATION OF CONVOLUTION AS FUSION}

Our strategy of proof of Theorem 1.10 is to show that both sides of (9) represent the same functor on the category $\widehat{\mathfrak{g}}_{\text {crit }}$-mod. This functor has to do with the notion of chiral pairing or fusion of modules over a chiral algebra, which we shall presently define. Having introduced this functor, in the rest of the section we consider various properties of fusion and its connection to the convolution functors.

2.1. Chiral pairings. Let $\mathcal{A}$ be a chiral algebra on a smooth curve $X$, and $\mathcal{M}_{1}, \mathcal{M}_{2}$ and $\mathcal{N}$ be chiral $\mathcal{A}$-modules. As usual, we shall denote by $\Delta$ the embedding of $X$ into $X^{n}$ as the main diagonal, and by $j$ the embedding of the complement of the diagonal divisor.

A chiral pairing $\left\{\mathcal{M}_{1}, \mathcal{M}_{2}\right\} \rightarrow \mathcal{N}$ is by definition a map of D-modules on $X \times X$ :

$$
\phi: j_{*} j^{*}\left(\mathcal{M}_{1} \otimes \mathcal{M}_{2}\right) \rightarrow \Delta_{!}(\mathcal{N}),
$$

which is compatible with the $\mathcal{A}$ action in the following sense:

We need that the sum of the three morphisms

$$
j_{*} j^{*}\left(\mathcal{A} \otimes \mathcal{M}_{1} \otimes \mathcal{M}_{2}\right) \rightarrow \Delta_{!}(\mathcal{N})
$$

is zero, where the first morphism is

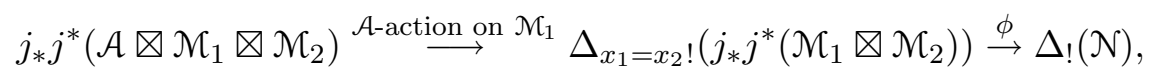

the second morphism is the negative of

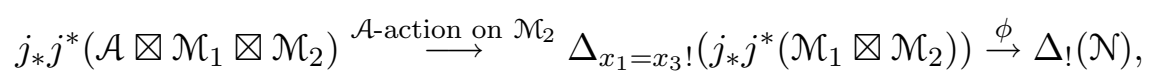

and the third morphism is

$$
j_{*} j^{*}\left(\mathcal{A} \otimes \mathcal{M}_{1} \otimes \mathcal{M}_{2}\right) \stackrel{\phi}{\rightarrow} \Delta_{x_{1}=x_{3} !}\left(j_{*} j^{*}(\mathcal{A} \otimes \mathcal{N})\right) \stackrel{\mathcal{A} \text {-action on } \mathcal{N}}{\longrightarrow} \Delta_{!}(\mathcal{N}) .
$$

Chiral pairings evidently form a functor $\mathcal{A}$-mod ${ }^{o} \times \mathcal{A}$-mod ${ }^{o} \times \mathcal{A}$-mod $\rightarrow$ Vect.

Remark. The notion of chiral pairing was introduced in [CHA]. As was mentioned in the introduction, in the case when $\mathcal{A}$ is obtained from a conformal vertex algebra $\mathrm{V}$, and the modules $\mathcal{M}_{1}, \mathcal{M}_{2}$ and $\mathcal{N}$ correspond to some $\mathrm{V}$-modules $M_{1}, M_{2}$ and $N$, the set of chiral pairings $\left\{\mathcal{M}_{1}, \mathcal{M}_{2}\right\} \rightarrow \mathcal{N}$ is in a natural bijection with the set of intertwining operators $M_{1} \otimes M_{2} \rightarrow N$ in the sense of [FHL], such that in the corresponding fields all powers of the formal variable $z$ are integral. 
Let us regard $\mathcal{A}$ as a chiral module over itself. Then for any other chiral $\mathcal{A}$ module $\mathcal{M}$, we have a canonical chiral pairing $\{\mathcal{A}, \mathcal{M}\} \rightarrow \mathcal{M}$ given by the action of $\mathcal{A}$ on $\mathcal{M}$. The following result is obtained directly from the definitions:

Lemma 2.2. The canonical chiral pairing $\{\mathcal{A}, \mathcal{M}\} \rightarrow \mathcal{M}$ establishes a bijection between the set of maps of chiral modules $\mathcal{M} \rightarrow \mathcal{N}$ and the set of chiral pairings $\{\mathcal{A}, \mathcal{M}\} \rightarrow \mathcal{N}$.

2.3. A canonical chiral pairing. Let $\kappa$ be any level, and let $\mathcal{A}_{\mathfrak{g}, \kappa}$ be the corresponding chiral algebra on $X$.

Consider the relative version $\operatorname{Gr}_{G, X}$ of the affine Grassmannian over $X$. Let $\mathcal{F}_{X}$ be a (right, $\kappa$-twisted) D-module on $\mathrm{Gr}_{G, X}$. In what follows we will assume that $\mathcal{F}_{X}$ is torsion-free with respect to $X$. By a slight abuse of notation, we will denote by $\Gamma\left(\operatorname{Gr}_{G, X}, \mathcal{F}_{X}\right)$ the quasi-coherent direct image of $\mathcal{F}_{X}$ on $X$; this is a chiral $\mathcal{A}_{\mathfrak{g}, \kappa}$-module. By replacing the subscript " $X$ " by either " $X-x$ " or " $x$ " we will denote the fibers of the above objects over $X-x$ and $x$ respectively.

Proposition-Construction 2.4. Given an object $\mathcal{M} \in \widehat{\mathfrak{g}}_{\kappa}-\bmod { }^{G[[t]]}$ there exists a canonical chiral pairing

$$
\left\{\Gamma\left(\mathrm{Gr}_{G, X}, \mathcal{F}_{X}\right), \mathcal{M}\right\} \rightarrow h^{0}\left(\mathcal{F}_{x} \star \mathcal{M}\right) .
$$

The rest of this subsection is devoted to the proof of this proposition.

Let $\operatorname{Jets}^{\mathrm{mer}}(G)_{X}$ be the D-ind scheme over $X$ of meromorphic jets into $G$. This is a relative version of the ind-scheme $G((t))$. By definition, the category $\mathrm{D}\left(\operatorname{Jets}^{\mathrm{mer}}(G)_{X}\right)_{\kappa}$-mod of (right $\kappa$-twisted) D-modules on $\operatorname{Jets}^{\operatorname{mer}}(G)_{X}$ is equivalent to that of chiral $\mathfrak{D}_{G, \kappa}$-modules.

We have a natural projection $\pi: \operatorname{Jets}^{\operatorname{mer}}(G)_{X} \rightarrow \operatorname{Gr}_{G, X}$ and by considering the quasi-coherent pull-back, we obtain a functor

$$
\pi^{*}: \mathrm{D}\left(\operatorname{Gr}_{G, X}\right)_{\kappa} \text {-mod } \rightarrow \mathrm{D}\left(\operatorname{Jets}^{\operatorname{mer}}(G)_{X}\right)_{\kappa} \text {-mod } .
$$

For $\mathcal{F}_{X} \in \mathrm{D}\left(\operatorname{Gr}_{G, X}\right)_{\kappa}$-mod, we will denote by $\Gamma\left(\operatorname{Jets}^{\operatorname{mer}}(G)_{X}, \pi^{*}\left(\mathcal{F}_{X}\right)\right)$ the resulting $\mathfrak{D}_{G, \kappa}$-module.

One reconstructs $\Gamma\left(\operatorname{Gr}_{G, X}, \mathcal{F}_{X}\right)$ as a subset of $\Gamma\left(\operatorname{Jets}^{\operatorname{mer}}(G)_{X}, \pi^{*}\left(\mathcal{F}_{X}\right)\right)$ as follows: this is the D-submodule consisting of sections that *-commute with $\mathfrak{r}\left(\mathcal{A}_{\mathfrak{g}, \kappa^{\prime}}\right)$, cf. [AG] or [FG1], Theorem 2.5.

For $\mathcal{F}_{X} \in \mathrm{D}\left(\operatorname{Gr}_{G, X}\right)_{\kappa}$-mod consider the action map

$$
j_{*} j^{*}\left(\Gamma\left(\operatorname{Jets}^{\text {mer }}(G)_{X}, \pi^{*}\left(\mathcal{F}_{X}\right)\right) \otimes \mathfrak{D}_{G, \kappa}\right) \rightarrow \Delta_{!}\left(\Gamma\left(\operatorname{Jets}^{\text {mer }}(G)_{X}, \pi^{*}\left(\mathcal{F}_{X}\right)\right)\right) .
$$


This is a $\mathfrak{D}_{G, \kappa^{-}}$chiral pairing, and hence, in particular, a chiral pairing with respect to the action of $\mathcal{A}_{\mathfrak{g}, \kappa}$ via l. In particular, we obtain a chiral $\mathcal{A}_{\mathfrak{g}, \kappa}$-pairing

$$
j_{*} j^{*}\left(\Gamma\left(\operatorname{Gr}_{G, X}, \mathcal{F}_{X}\right) \otimes \mathfrak{D}_{G, \kappa}\right) \rightarrow \Delta_{!}\left(\Gamma\left(\operatorname{Jets}^{\operatorname{mer}}(G)_{X}, \pi^{*}\left(\mathcal{F}_{X}\right)\right) .\right.
$$

This map commutes with the right $\mathcal{A}_{\mathfrak{g}, \kappa^{\prime}}$-action in the sense that the map

$$
\begin{aligned}
& \widetilde{j}_{*} \widetilde{j}^{*}\left(\Gamma\left(\operatorname{Gr}_{G, X}, \mathcal{F}_{X}\right) \otimes \mathfrak{D}_{G, \kappa} \otimes \mathcal{A}_{\mathfrak{g}, \kappa^{\prime}}\right) \rightarrow \Delta_{x_{1}=x_{2} !}\left(\Gamma\left(\operatorname{Jets}^{\operatorname{mer}}(G)_{X}, \pi^{*}\left(\mathcal{F}_{X}\right)\right) \otimes \mathcal{A}_{\mathfrak{g}, \kappa^{\prime}}\right) \rightarrow \\
& \rightarrow \Delta_{!}\left(\Gamma\left(\operatorname{Jets}^{\operatorname{mer}}(G)_{X}, \pi^{*}\left(\mathcal{F}_{X}\right)\right)\right)
\end{aligned}
$$

coincides with

$$
\begin{aligned}
& \widetilde{j}_{*} \widetilde{j}^{*}\left(\Gamma\left(\operatorname{Gr}_{G, X}, \mathcal{F}_{X}\right) \otimes \mathfrak{D}_{G, \kappa} \otimes \mathcal{A}_{\mathfrak{g}, \kappa^{\prime}}\right) \rightarrow \Delta_{x_{2}=x_{3} !}\left(\Gamma\left(\operatorname{Gr}_{G, X}, \mathcal{F}_{X}\right) \otimes \mathfrak{D}_{G, \kappa}\right) \rightarrow \\
& \rightarrow \Delta_{!}\left(\Gamma\left(\operatorname{Jets}^{\mathrm{mer}}(G)_{X}, \pi^{*}\left(\mathcal{F}_{X}\right)\right)\right),
\end{aligned}
$$

where $\tilde{j}$ denotes the embedding of the complement of the union of divisors corresponding to $x_{1}=x_{2}$ and $x_{2}=x_{3}$.

Let us restrict both sides of (20) to $X \times x \subset X \times X$. We obtain a chiral pairing

$$
j_{x *} j_{x}^{*}\left(\Gamma\left(\operatorname{Gr}_{G, X-x}, \mathcal{F}_{X-x}\right)\right) \otimes \mathfrak{D}_{G, \kappa, x} \rightarrow i_{x !}\left(\Gamma\left(G((t)), \pi^{*}\left(\mathcal{F}_{x}\right)\right)\right),
$$

where we view $\mathcal{F}_{x}$ as a $\kappa$-twisted D-module over the affine Grassmannian, and $i_{x}$ (resp., $j_{x}$ ) denotes the embedding of $x$ into $X$ (resp., the embedding of this complement).

By the above, the map in (21) commutes with the action of $\widehat{\mathfrak{g}}_{\kappa^{\prime}}$ via $\mathfrak{r}$. (On the LHS this action affects only the $\mathfrak{D}_{G, \kappa, x}$ multiple.)

Let now $\mathcal{M}$ be a $G[[t]]$-integrable $\widehat{\mathfrak{g}}_{\kappa}$-module. By considering the complex, computing the semi-infinite Tor,

$$
\mathfrak{C}^{\frac{\infty}{2}}(\mathfrak{g}((t)) ; \mathfrak{g}, ? \otimes \mathcal{M})
$$

against the two sides of $(21)$, considered as $\widehat{\mathfrak{g}}_{\kappa^{\prime}}$-modules, we obtain a chiral pairing of complexes of $\mathcal{A}_{\mathfrak{g}, \kappa}$-modules:

$\left\{\Gamma\left(\operatorname{Gr}_{G, X}, \mathcal{F}_{X}\right), \mathfrak{C}^{\frac{\infty}{2}}\left(\mathfrak{g}((t)) ; \mathfrak{g}, \mathfrak{D}_{G, \kappa, x} \otimes \mathcal{M}\right)\right\} \rightarrow \mathfrak{C}^{\frac{\infty}{2}}\left(\mathfrak{g}((t)) ; \mathfrak{g}, \Gamma\left(G((t)), \pi^{*}\left(\mathcal{F}_{x}\right)\right) \otimes \mathcal{M}\right)$.

By passing to the 0th cohomology, and taking into account (14) we obtain the chiral pairing of (18). 
2.5. Chiral pairing with a module of global sections is represented by the convolution. Let us now specialize to the case when $\kappa$ is integral nonpositive (see Sect. 1.1 for the definition of what this means). For $V \in \operatorname{Rep}(\check{G})$, let $\mathcal{F}_{V, X}$ be the corresponding object of $\mathrm{D}\left(\mathrm{Gr}_{G, X}\right)_{\kappa}$-mod. Then from (18) we obtain a canonical chiral pairing

$$
\left\{\Gamma\left(\operatorname{Gr}_{G, X}, \mathcal{F}_{V, X}\right), \mathcal{M}\right\} \rightarrow h^{0}\left(\mathcal{F}_{V} \star \mathcal{M}\right) .
$$

In Sect. 4 we will prove the following theorem:

\section{Theorem 2.6.}

(1) For any $\mathcal{M} \in \widehat{\mathfrak{g}}_{\kappa}-\bmod ^{G[[t]]}$ and $V \in \operatorname{Rep}(\check{G})$, the convolution $\mathcal{F}_{V} \star \mathcal{M}$ is acyclic away from cohomological degree 0 .

(2) The functor on $\widehat{\mathfrak{g}}_{\kappa}$-mod that sends $\mathcal{N}$ to the set of chiral parings $\left\{\Gamma\left(\mathrm{Gr}_{G, X}, \mathcal{F}_{V, X}\right), \mathcal{M}\right\} \rightarrow \mathcal{N}$ is representable by $\mathcal{F}_{V} \star \mathcal{M}$.

Our proof of Theorem 1.10 will be independent of Theorem 2.6. However, it is useful to keep Theorem 2.6 in mind as it gives us an important insight into the connection between fusion and convolution.

2.7. We will need the following result about the associativity property of the map given by $(22)$.

For any two objects $V, W \in \operatorname{Rep}(\check{G})$ there exists a canonical chiral pairing

$$
\left\{\Gamma\left(\operatorname{Gr}_{G, X}, \mathcal{F}_{V, X}\right), \Gamma\left(\operatorname{Gr}_{G, X}, \mathcal{F}_{W, X}\right)\right\} \rightarrow \Gamma\left(\operatorname{Gr}_{G, X}, \mathcal{F}_{V \otimes W, X}\right) .
$$

(Its construction will be recalled in the sequel.) Let $\mathcal{M}$ be an object of $\mathcal{M} \in$ $\widehat{\mathfrak{g}}_{\kappa^{-} \text {-mod }}{ }^{G[t]]}$, such that $\mathcal{F}_{V^{\prime}} \star \mathcal{M}$ is acyclic away from cohomological degree 0 for any $V^{\prime} \in \operatorname{Rep}(\check{G})$ (we choose not to rely here on Theorem 2.6(1), which says that the latter assumption is satisfied automatically).

Then there are three maps

$$
j_{*} j^{*}\left(\Gamma\left(\mathrm{Gr}_{G, X}, \mathcal{F}_{V, X}\right) \otimes \Gamma\left(\mathrm{Gr}_{G, X}, \mathcal{F}_{W, X}\right) \otimes \mathcal{M}\right) \rightarrow \Delta_{!}\left(\mathcal{F}_{V \otimes W} \star \mathcal{M}\right):
$$

The first one is the composition

$$
\begin{aligned}
& j_{*} j^{*}\left(\Gamma\left(\operatorname{Gr}_{G, X}, \mathcal{F}_{V, X}\right) \otimes \Gamma\left(\operatorname{Gr}_{G, X}, \mathcal{F}_{W, X}\right) \otimes \mathcal{M}\right) \rightarrow \\
& \rightarrow \Delta_{x_{2}=x_{3} !}\left(j_{*} j^{*}\left(\Gamma\left(\operatorname{Gr}_{G, X}, \mathcal{F}_{V, X}\right) \otimes\left(\mathcal{F}_{W} \star \mathcal{M}\right)\right)\right) \rightarrow \\
& \rightarrow \Delta_{!}\left(\mathcal{F}_{V} \star\left(\mathcal{F}_{W} \star \mathcal{M}\right)\right) \simeq \Delta_{!}\left(\mathcal{F}_{V \otimes W} \star \mathcal{M}\right) .
\end{aligned}
$$


The second map is the negative of a similar map with the roles of the first and the second factor swapped. The third map is the composition

$$
\begin{aligned}
& j_{*} j^{*}\left(\Gamma\left(\operatorname{Gr}_{G, X}, \mathcal{F}_{V, X}\right) \otimes \Gamma\left(\operatorname{Gr}_{G, X}, \mathcal{F}_{W, X}\right) \otimes \mathcal{M}\right) \rightarrow \\
& \rightarrow \Delta_{x_{1}=x_{2} !}\left(j_{*} j^{*}\left(\Gamma\left(\operatorname{Gr}_{G, X}, \mathcal{F}_{V \otimes W, X}\right) \otimes \mathcal{M}\right)\right) \rightarrow \Delta_{!}\left(\mathcal{F}_{V \otimes W} \star \mathcal{M}\right) .
\end{aligned}
$$

Proposition 2.8. The sum of the above three maps is 0 .

2.9. Proof of Proposition 2.8. By the construction of the chiral pairing (22), it is sufficient to consider the universal case, i.e., when $\mathcal{M} \simeq \mathfrak{D}_{G, \kappa}$ as a chiral $\mathcal{A}_{\mathfrak{g}, \kappa}$-module under $\mathfrak{l}$.

Let us rewrite the map (19) in more geometric terms. For any $n$, let $\operatorname{Gr}_{G, X^{n}}$ be the Beilinson-Drinfeld affine Grassmannian over $X^{n}$. I.e., this is the ind-scheme classifying the data of

$$
\left(x_{1}, \ldots, x_{n}, \mathcal{P}_{G}, \beta\right)
$$

where $x_{1}, \ldots, x_{n}$ is an $n$-tuple of points on $X, \mathcal{P}_{G}$ is a principal $G$-bundle on $X$, and $\beta$ is a trivialization of $\mathcal{P}_{G}$ on $X-\left\{x_{1}, \ldots, x_{n}\right\}$.

For a decomposition $n=n_{1}+n_{2}$ there exists a natural map

$$
\mathbf{1}_{n_{1}, n_{2}}: X^{n_{1}} \times \operatorname{Gr}_{G, X^{n_{2}}} \rightarrow \operatorname{Gr}_{G, X^{n}}
$$

which sends $\left(\left(x_{1}, \ldots x_{n_{1}}\right),\left(x_{n_{1}+1}, \ldots, x_{n}, \mathcal{P}_{G}, \beta\right)\right) \mapsto\left(x_{1}, \ldots, x_{n_{1}}, x_{n_{1}+1}, \ldots, x_{n}, \mathcal{P}_{G}, \beta\right)$.

Consider now the ind-scheme Jets ${ }^{\operatorname{mer}}(G)_{X^{n}}$, fibered over $\operatorname{Gr}_{G, X^{n}}$, where in addition to the data $\left(x_{1}, \ldots, x_{n}, \mathcal{P}_{G}, \beta\right)$ we have that of a trivialization of $\mathcal{P}_{G}$ on a formal neighborhood of $\cup_{i} x_{i} \subset X$. Let us denote by $\pi$ the corresponding projection. This ind-scheme also carries a flat connection along $X^{n}$.

The scheme $\operatorname{Jets}^{\mathrm{mer}}(G)_{X^{n}}$ has the usual factorization pattern:

$$
j^{*}\left(\operatorname{Jets}^{\operatorname{mer}}(G)_{X^{n}}\right) \simeq j^{*}\left(\operatorname{Jets}^{\operatorname{mer}}(G)_{X}^{\times n}\right) \text { and } \Delta^{*}\left(\operatorname{Jets}^{\operatorname{mer}}(G)_{X^{n}}\right) \simeq \operatorname{Jets}^{\text {mer }}(G)_{X} \text {. }
$$

For two objects $V, W \in \operatorname{Rep}(\check{G})$ we consider the D-module $j^{*}\left(\mathcal{F}_{V, X} \otimes \mathcal{F}_{W, X}\right)$ on $\left.\operatorname{Gr}_{G, X^{2}}\right|_{X^{2}-X}$. As is well-known, its Goresky-MacPherson extension $j_{! *}\left(j^{*}\left(\mathcal{F}_{V, X} \otimes \mathcal{F}_{W, X}\right)\right)$ onto $\operatorname{Gr}_{G, X^{2}}$ has the property that

$$
\Delta^{!}\left(j_{! *}\left(j^{*}\left(\mathcal{F}_{V, X} \otimes \mathcal{F}_{W, X}\right)\right)\right)[1] \simeq \mathcal{F}_{V \otimes W, X} .
$$

Consider the (right $\kappa$-twisted) D-module

$$
\left(\mathbf{1}_{1,2}\right) !\left(\omega_{X} \otimes j_{! *}\left(j^{*}\left(\mathcal{F}_{V, X} \otimes \mathcal{F}_{W, X}\right)\right)\right)
$$

on $\mathrm{Gr}_{G, X^{3}}$. We have three maps 


$$
j_{*} j^{*}\left(\left(\mathbf{1}_{1,2}\right) !\left(j_{! *}\left(\omega_{X} \otimes j^{*}\left(\mathcal{F}_{V, X} \otimes \mathcal{F}_{W, X}\right)\right)\right)\right) \rightarrow \Delta_{!}\left(\mathcal{F}_{V \otimes W, X}\right),
$$

corresponding to the three diagonals in $X^{3}$, whose sum is equal to 0 .

Applying the pull-back by means of $\pi$ to the two sides of the above formula to Jets $^{\operatorname{mer}}(G)_{X^{3}}$, followed by the direct image onto $X^{3}$, we obtain three maps

$$
\begin{aligned}
& j_{*} j^{*}\left(\mathfrak{D}_{G, \kappa} \otimes \Gamma\left(\operatorname{Jets}^{\mathrm{mer}}(G)_{X}, \pi^{*}\left(\mathcal{F}_{V, X}\right)\right) \otimes \Gamma\left(\operatorname{Jets}^{\text {mer }}(G)_{X}, \pi^{*}\left(\mathcal{F}_{W, X}\right)\right)\right) \rightarrow \\
& \rightarrow \Delta_{!}\left(\Gamma\left(\operatorname{Jets}^{\text {mer }}(G)_{X}, \pi^{*}\left(\mathcal{F}_{V \otimes W}\right)\right) .\right.
\end{aligned}
$$

Finally, note that we have a natural inclusion

$$
\begin{aligned}
& j_{*} j^{*}\left(\mathfrak{D}_{G, \kappa} \otimes \Gamma\left(\operatorname{Gr}_{G, X}, \mathcal{F}_{V, X}\right) \otimes \Gamma\left(\operatorname{Gr}_{G, X}, \mathcal{F}_{W, X}\right)\right) \hookrightarrow \\
& j_{*} j^{*}\left(\mathfrak{D}_{G, \kappa} \otimes \Gamma\left(\operatorname{Jets}^{\text {mer }}(G)_{X}, \pi^{*}\left(\mathcal{F}_{V, X}\right)\right) \otimes \Gamma\left(\operatorname{Jets}^{\operatorname{mer}}(G)_{X}, \pi^{*}\left(\mathcal{F}_{W, X}\right)\right)\right) .
\end{aligned}
$$

By composing this inclusion with the three maps above we obtain three maps (24)

$j_{*} j^{*}\left(\mathfrak{D}_{G, \kappa} \otimes \Gamma\left(\operatorname{Gr}_{G, X}, \mathcal{F}_{V, X}\right) \otimes \Gamma\left(\operatorname{Gr}_{G, X}, \mathcal{F}_{W, X}\right)\right) \rightarrow \Delta_{!}\left(\Gamma\left(\operatorname{Jets}^{\operatorname{mer}}(G)_{X}, \pi^{*}\left(\mathcal{F}_{V \otimes W, X}\right)\right)\right)$.

Lemma 2.10. The three maps of (24) coincide with those that appear in the statement of Proposition 2.8.

Clearly, this implies the statement of the proposition. To prove the above lemma we will consider a slightly more general framework.

2.11. Let $\mathcal{F}_{1}$ be a ( $\kappa$-twisted, right) D-module on $G((t)) / K$, where $K \subset G[[t]]$ is a subgroup of finite codimension. Let $\mathcal{F}_{1}^{\prime}$ be the pull-back of $\mathcal{F}_{1}$ to $G((t))$.

Consider the ind-scheme $\operatorname{Gr}_{G, X ; K}$, classifying the data $\left(x_{1}, \mathcal{P}_{G}, \beta, \alpha\right)$, where $\left(x_{1}, x, \mathcal{P}_{G}, \beta\right)$ is a point of $\operatorname{Gr}_{G, X \times x}$, and $\alpha$ is a reduction of $\left.\mathcal{P}_{G}\right|_{\mathcal{D}_{x}}$ to $K$. We have the isomorphisms

$$
\mathrm{Gr}_{G, X-x ; K} \simeq \operatorname{Gr}_{G, X-x} \times G((t)) / K \text { and } \operatorname{Gr}_{G, x ; K} \simeq G((t)) / K .
$$

We also have a natural projection $\pi_{K}: \operatorname{Jets}^{\operatorname{mer}}(G)_{X \times x} \rightarrow \operatorname{Gr}_{G, X ; K}$, where Jets $^{\text {mer }}(G)_{X \times x}$ denotes the preimage of $X \times x \subset X^{2}$ in $\operatorname{Jets}^{\text {mer }}(G)_{X^{2}}$.

Consider the direct image of $\omega_{X} \otimes \mathcal{F}_{1}$ under the tautological embedding $\mathbf{1}_{1,1}$ : $X \times G((t)) / K \rightarrow \mathrm{Gr}_{G, X ; K}$. It gives rise to a map of twisted D-modules on $\mathrm{Gr}_{G, X ; K}$ :

$$
j_{x *} j_{x}^{*}\left(\delta_{1, \operatorname{Gr}_{G, X}} \otimes \mathcal{F}_{1}\right) \rightarrow i_{x !}\left(\mathcal{F}_{1}\right) .
$$


Lifting the two sides of (25) by means of $\pi_{K}$ and taking the (quasi-coherent direct image onto $X \simeq X \times x$, we obtain a map

$$
j_{x *} j_{x}^{*}\left(\mathfrak{D}_{G, \kappa}\right) \otimes \Gamma\left(G((t)), \mathcal{F}_{1}^{\prime}\right) \rightarrow i_{x !}\left(\Gamma\left(G((t)), \mathcal{F}_{1}^{\prime}\right)\right) .
$$

The following assertion is built into the interpretation of twisted D-modules on $G\left(((t))\right.$ as chiral $\mathfrak{D}_{G, \kappa}$-modules:

Proposition 2.12. The map of (26) coincides with the chiral action map for $\Gamma\left(G((t)), \mathcal{F}_{1}^{\prime}\right)$ considered as a chiral $\mathfrak{D}_{G, \kappa}$-module supported at $x$.

In the appendix (Sect. 8) we shall give a proof of this result for the sake of completeness.

Assume now that the D-module $\mathcal{F}_{1}$ on $G((t)) / K$ is $G[[t]]$-equivariant with respect to the left action of $G[[t]]$ on $G((t)) / K$. Let now $\mathcal{F}_{X}$ be a ( $\kappa$-twisted, right) D-module on $\operatorname{Gr}_{G, X}$. In this case we can form the convolution $\mathcal{F}_{X} \underset{G[t]]}{\star} \mathcal{F}_{1}$, which will be an object of the derived category of ( $\kappa$-twisted, right) D-modules on $\operatorname{Gr}_{G, X ; K}$. We have:

$$
\left.\mathcal{F}_{X} \underset{G[[t]]}{\star} \mathcal{F}_{1}\right|_{G_{G, X-x ; K}} \simeq \mathcal{F}_{X-x} \otimes \mathcal{F}_{1} \text { and }\left.\mathcal{F}_{x} \underset{G[[t]]}{\star} \mathcal{F}_{1}\right|_{G_{G, x ; K}} \simeq \mathcal{F}_{x} \underset{G[[t]]}{\star} \mathcal{F}_{1} .
$$

In particular, we obtain a map of D-modules on $\operatorname{Gr}_{G, X ; K}$ :

$$
j_{x *} j_{x}^{*}\left(\mathcal{F}_{X-x} \otimes \mathcal{F}_{1}\right) \rightarrow i_{x !}\left(h^{0}\left(\mathcal{F}_{x}^{\star} \underset{G[t]]}{\star} \mathcal{F}_{1}\right)\right) .
$$

Pulling the two sides of (27) under the map $\pi_{K}: \operatorname{Jets}^{\mathrm{mer}}(G)_{X \times x} \rightarrow \operatorname{Gr}_{G, X ; K}$, and taking the (quasi-coherent) direct image onto $X$, we obtain a map of D-modules on $X$ :

$$
\begin{aligned}
& j_{x *} j_{x}^{*}\left(\Gamma\left(\operatorname{Jets}^{\text {mer }}(G)_{X}, \pi^{*}\left(\mathcal{F}_{X}\right)\right)\right) \otimes \Gamma\left(G((t)), \mathcal{F}_{1}^{\prime}\right) \rightarrow i_{x !}\left(\Gamma\left(G((t)), h^{0}\left(\mathcal{F}_{x} \star \mathcal{F}_{1}^{\prime}\right)\right)\right) \simeq \\
& \simeq i_{x !}\left(h^{0}\left(\mathcal{F}_{x} \star \Gamma\left(G((t)), \mathcal{F}_{1}^{\prime}\right)\right)\right) .
\end{aligned}
$$

Lemma 2.13. The above map coincides with (22) for $\mathcal{M}=\Gamma\left(G((t)), \mathcal{F}_{1}^{\prime}\right)$.

It is easy to see that this lemma, combined with a version of Proposition 2.12, where the point $x$ varies on $X$, imply the required property of the three maps of (24).

Proof. (of Lemma 2.13)

Consider first the case when $K=G[[t]]$ and $\mathcal{F}_{1}=\delta_{1, \mathrm{Gr}_{G}}$. Then we are dealing with a map

$$
j_{x *} j_{x}^{*}\left(\Gamma\left(\operatorname{Jets}^{\mathrm{mer}}(G)_{X}, \pi^{*}\left(\mathcal{F}_{X}\right)\right)\right) \otimes \mathfrak{D}_{G, \kappa, x} \rightarrow i_{x !}\left(\Gamma\left(G((t)), \pi^{*}\left(\mathcal{F}_{x}\right)\right)\right),
$$


and we claim that it coincides with the map, obtained by restriction to $X \times x$ from the map of (19). This follows from a version of Proposition 2.12 for $K=G[[t]]$, when instead of the fixed point $x$ we consider a morphism of D-modules on $X \times X$, and the map (19) itself is described as a Cousin map corresponding to $\left(\mathbf{1}_{1,1}\right)_{!}\left(\mathcal{F}_{X}\right)$, viewed as D-module on $\mathrm{Gr}_{G, X^{2}}$.

To pass to the general case, consider the category of $\kappa$-twisted D-modules on Jets $^{\operatorname{mer}}(G)_{X, x}$ as acted on by the group ind-scheme $G((t))$ on the right, which is of Harish-Chandra type with respect to the extension $\widehat{\mathfrak{g}}_{\kappa^{\prime}}$ of $\mathfrak{g}((t))$. Then the convolution action of $\mathcal{F}_{1}$ on

$$
j_{* *} j_{x}^{*}\left(\pi^{*}\left(\mathcal{F}_{X-x}\right) \otimes \pi^{*}\left(\delta_{1, \mathrm{Gr}_{G}}\right)\right)\left(\text { resp., } i_{x !}\left(\mathcal{F}_{x}\right)\right)
$$

equals

$$
j_{* *} j_{x}^{*}\left(\pi^{*}\left(\mathcal{F}_{X-x}\right) \otimes \mathcal{F}_{1}^{\prime}\right) \text { and } i_{x !}\left(\mathcal{F}_{x} \star \mathcal{F}_{1}^{\prime}\right),
$$

respectively.

Consider also the category of D-modules on $X$, endowed with an action of $\widehat{\mathfrak{g}}_{\kappa^{\prime}}$ as a category with a Harish-Chandra action of $G((t))$. Then the convolution action of $\mathcal{F}_{1}$ on a module of the type $j_{x *} j_{x}^{*}(\mathcal{M}) \otimes \mathfrak{D}_{G, \kappa, x}$ (resp., $i_{x !}(\mathcal{N})$ ) (where $\mathcal{M}$ is a $\mathrm{D}$-module, and $\mathcal{N}$ is a representation of $\widehat{\mathfrak{g}}_{\kappa^{\prime}}$ ) equals

$$
j_{x *} j_{x}^{*}(\mathcal{N}) \otimes \Gamma\left(G((t)), \mathcal{F}_{1}^{\prime}\right) \text { and } i_{x !}\left(\mathfrak{C}^{\frac{\infty}{2}}\left(\mathfrak{g}((t)) ; \mathfrak{g}, \mathcal{N} \otimes \Gamma\left(G((t)), \mathcal{F}_{1}^{\prime}\right)\right)\right),
$$

respectively.

Note now that the (quasi-coherent) direct image is a functor between the above two categories that respects the $G((t))$-actions. Hence, the assertion of the lemma follows from the case when $K=G[[t]]$ and $\mathcal{F}_{1}=\delta_{1, \mathrm{Gr}_{G}}$ by the functoriality of the convolution.

\section{Proof of Theorem 1.10}

Having established some preliminary results in the previous section, we are now ready to prove Theorem 1.10. The plan of the proof is as follows: we will first show that the chiral maps $\left\{\mathcal{M}_{1}, \mathcal{M}\right\} \rightarrow \mathcal{N}$ in the special case when $\mathcal{M}_{1}=\mathcal{V}_{\mathfrak{z} \mathfrak{g}} \otimes \mathcal{A}_{\mathfrak{g} \mathfrak{g} \text {,crit }}$ are the same as ordinary homomorphisms of $\widehat{\mathfrak{g}}_{\text {crit }}$-modules $\mathcal{M}^{\prime} \rightarrow \mathcal{N}$ where $\mathcal{M}^{\prime} \simeq \mathcal{V}_{\widetilde{\mathfrak{Z}}_{\mathfrak{g}}^{\text {int,reg }}} \underset{\widetilde{\mathfrak{Z}}_{\mathfrak{g}}^{\text {int,reg }}}{\otimes} \mathcal{M}$. Roughly speaking, this means that we can "swap", under the chiral product, the tensor product with $\mathcal{V}_{\mathfrak{z} \mathfrak{g}}$ from $\mathcal{A}_{\mathfrak{g}, \text { crit }}$ to $\mathcal{M}$. This is the content of Theorem 3.2.

Once we have that, we obtain, for each $V \in \operatorname{Rep}(\check{G})$, a map in one direction in (9), provided that $\mathcal{F}_{V} \star \mathcal{M}$ is acyclic away from cohomological dimension 0 . 
But we do know this for $\mathcal{M}=\mathfrak{D}_{G, \text { crit, } x}$, according to Theorem 1.13. Moreover, according to Sect. 1.12, it is sufficient to prove the statement of Theorem 1.10 just in this special case. Hence all we need is to check that the maps we have constructed in this case are indeed isomorphisms and that they satisfy the properties listed in Theorem 1.10. We first check that the maps in question do satisfy the required properties; then we show that this already implies that the above maps are necessarily isomorphisms, thus completing the proof.

3.1. Fusion at the critical level. In this section we fix $\kappa=\kappa_{\text {crit }}$, and study the map of (22) in this case. We will use the following crucial result of [BD], Sects. 5.5-5.6: there is a canonical isomorphism of chiral $\mathcal{A}_{\mathfrak{g}, \text { crit-modules, }}$

$$
\Gamma\left(\operatorname{Gr}_{G, X}, \mathcal{F}_{V, X}\right) \simeq \mathcal{V}_{\mathfrak{z} \mathfrak{g}} \underset{\mathfrak{z} \mathfrak{g}}{\otimes} \mathcal{A}_{\mathfrak{g}, \text { crit }},
$$

where the tensor product makes sense since $\mathfrak{z}_{\mathfrak{g}}$ is the center of $\mathcal{A}_{\mathfrak{g} \text {,crit }}$.

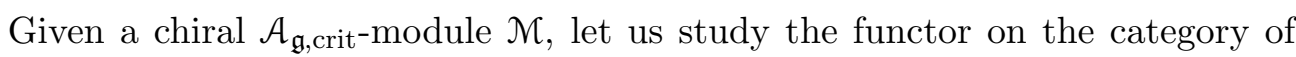
chiral $\mathcal{A}_{\mathfrak{g}, \text { crit }}$-modules that assigns to a chiral $\mathcal{A}_{\mathfrak{g}, \text { crit-module }} \mathcal{N}$ the set of chiral pairings

$$
\left\{\mathcal{V}_{\mathfrak{z} \mathfrak{g}} \otimes \mathcal{A}_{\mathfrak{g} \mathfrak{g}, \text { crit }}, \mathcal{M}\right\} \rightarrow \mathcal{N}
$$

Theorem 3.2. Let $\mathcal{M}$ be concentrated at $x \in X$, and assume that, when viewed as a module over $\mathfrak{Z}_{\mathfrak{g}}$, it is supported on $\widetilde{\mathfrak{Z}}_{\mathfrak{g}}^{\mathrm{int}, \mathrm{reg}}$. Then chiral pairings (29) for $\mathcal{N} \in \mathcal{A}_{\mathfrak{g}, \text { crit }}-$ mod $_{x}$ are in bijection with maps of $\widehat{\mathfrak{g}}_{\text {crit }}$-modules

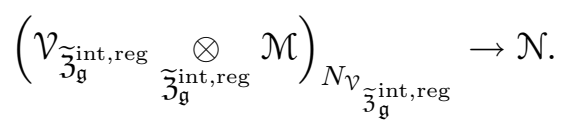

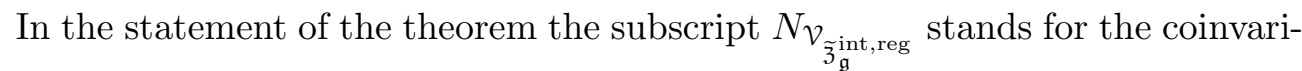
ants with respect to the action of this nilpotent operator. Before giving a proof of this theorem, which will occupy the next few subsections, let us note that Theorem 3.2 implies the existence of the map in one direction in Theorem 1.10:

Indeed, the chiral pairing (22) gives rise to a map

$$
\left(\mathcal{V}_{\widetilde{\mathfrak{Z}}_{\mathfrak{g}}^{\text {int,reg }}} \underset{\widetilde{\mathfrak{Z}}_{\mathfrak{g}}^{\text {int,reg }}}{\otimes} \mathcal{M}\right)_{N_{\mathcal{\mathfrak { J }}_{\mathfrak{g}}^{\text {int,reg }}}} \rightarrow h^{0}\left(\mathcal{F}_{V} \star \mathcal{M}\right) .
$$

Note that if we assume Theorem 2.6, then we obtain that the map of (30) is an isomorphism (and moreover, that $\left.h^{0}\left(\mathcal{F}_{V} \star \mathcal{M} \mathcal{N}\right)=\mathcal{F}_{V} \star \mathcal{M}\right)$. However, this would still not be enough to prove Theorem 1.10: we would also need to show that $N_{\mathcal{V}_{\widetilde{\mathfrak{z}}_{\mathfrak{g}} \text { int,reg }}}$ acts trivially on the left-hand side, which requires a separate argument. The proof of Theorem 1.10 that we give below will be independent of Theorem 2.6. 
3.3. Fusion over commutative chiral algebras. To prove Theorem 3.2 we need to study chiral pairings between modules over commutative chiral algebras.

Recall the set-up of the proof of Proposition-Construction 1.3, i.e., let $\mathcal{B}$ be a commutative chiral algebra on $X$, and let $\mathcal{V}_{X-x}$ be a ${ }^{*}$-commutative module over $\left.\mathcal{B}\right|_{X-x}$, extended to the entire $X$ such that the connection has a pole of order $\leq 1$ and the residue $\operatorname{Res}\left(\nabla, \mathcal{V}_{X}\right) \in \operatorname{End}\left(\mathcal{V}_{x}\right)$ is nilpotent.

Proposition 3.4. Let $\mathcal{M}$ be a $\mathcal{B}_{x}$-module, viewed as ${ }^{*}$-commutative $\mathcal{B}$-module, supported at $x$. Let $\mathcal{N}$ be another chiral $\mathcal{B}$-module, supported at $x$. Then chiral pairings

$$
\left\{\mathcal{V}_{X-x}, \mathcal{M}\right\} \rightarrow \mathcal{N}
$$

are in bijection with maps of $\hat{\mathcal{B}}_{x}$-modules

$$
\left(\mathcal{V}_{x} \underset{\mathcal{B}_{x}}{\otimes} \mathcal{M}\right)_{\operatorname{Res}\left(\nabla, \mathcal{V}_{X}\right)} \rightarrow \mathcal{N}
$$

where $\hat{\mathcal{B}}_{x}$ denotes the topological commutative algebra, corresponding to $\mathcal{B}$ at $x$.

Proof. First, from the definition of chiral pairings it is easy to see that any pairing $\left\{\mathcal{V}_{X-x}, \mathcal{N}\right\} \rightarrow \mathcal{N}$ factors through $\mathcal{N}^{\prime} \subset \mathcal{N}$, where $\mathcal{N}^{\prime}$ is the maximal submodule of $\mathcal{N}$ on which $\hat{\mathcal{B}}_{x}$ acts via $\hat{\mathcal{B}}_{x} \rightarrow \mathcal{B}_{x}$. In other words, we can assume that $\mathcal{N}$ is also *-commutative.

Secondly, for a fixed $\mathcal{N}$, both (31) and (32), regarded as contravariant functors with respect to $\mathcal{M}$ are both right exact and commute with direct limits. Hence, we can replace $\mathcal{M}$ by $\mathcal{B}_{x}$.

Let us denote by $B$ the fiber $\mathcal{B}_{x}$ and by $T_{0}$ the module over $B[[t]]$, corresponding to $\mathcal{V}_{X}$. Let $T$ be the localization of $T_{0}$ with respect to $t$.

Then chiral pairings $\left\{\mathcal{V}_{X-x}, \mathcal{B}_{x}\right\} \rightarrow \mathcal{N}$ are in bijection with $B[[t]]$-linear maps

$$
\phi: T \rightarrow \underset{B}{\otimes} B((t)) d t / B[[t]] d t,
$$

that respect that action of $\partial_{t}$.

Given such $\phi$, the finite rank assumption on $T_{0}$ implies that for some integer $k$ the composition

$$
t^{k} \cdot T_{0} \hookrightarrow T \stackrel{\phi}{\rightarrow} \underset{\mathcal{N}}{\otimes} \underset{B}{B} B((t)) / B[[t]]
$$

is zero. However, the nilpotency assumption on $t \partial_{t}$ acting on $T_{0} / t \cdot T_{0}$ implies that for any $k>0$ the map

$$
\partial_{t}:\left(t^{k} \cdot T_{0}\right) \rightarrow\left(t^{k-1} \cdot T_{0}\right)
$$

is surjective. 
Therefore, $\phi$ in fact annihilates $T_{0}$. Restricting $\phi$ to $t^{-1} \cdot T_{0}$ we obtain a map

$$
t^{-1} \cdot T_{0} / T_{0} \rightarrow\left(t^{-1} \mathbb{C}[[t]] / \mathbb{C}[[t]]\right) \otimes \mathcal{N},
$$

which is zero on the image of $\partial_{t}\left(T_{0}\right)$. Hence, we obtain a map

$$
\operatorname{coker}\left(t \partial_{t}: T_{0} / t \cdot T_{0} \rightarrow T_{0} / t \cdot T_{0}\right) \rightarrow \mathcal{N}
$$

as desired.

Conversely, starting from a map as in(34), it is easy to see that it uniquely extends to a map as in (33).

Let us denote by canv the resulting canonical map

$$
j_{*} j^{*}\left(\mathcal{V}_{X-x} \otimes \mathcal{M}\right) \rightarrow \Delta_{!}\left(\mathcal{V}_{x} \underset{\mathcal{B}_{x}}{\otimes} \mathcal{M}\right)_{\operatorname{Res}\left(\nabla, \mathcal{V}_{X}\right)}
$$

For the proof of Theorem 1.10, we will need the following additional property of this map.

Let $\left(\mathcal{V}_{X-x}, \mathcal{V}_{X}\right)$ and $\left(\mathcal{W}_{X-x}, \mathcal{W}_{X}\right)$ be two pairs of $\mathcal{B}$-modules, satisfying the assumptions of Proposition 3.4. Note that the tensor product $\left(\mathcal{V}_{X-x} \underset{\mathcal{B}_{X-x}}{\otimes} \mathcal{W}_{X-x}, \mathcal{V}_{X} \underset{\mathcal{B}}{\otimes} \mathcal{W}_{X}\right)$ also has the same property.

We have three maps

$$
j_{*} j^{*}\left(\mathcal{V}_{X-x} \otimes \mathcal{W}_{X-x} \nabla \mathcal{M}\right) \rightarrow \Delta_{!}\left(\mathcal{V}_{x} \underset{\mathcal{B}_{x}}{\otimes} \mathcal{W}_{x} \underset{\mathcal{B}_{x}}{\otimes} \mathcal{M}\right)_{\operatorname{Res}\left(\nabla, \mathcal{V}_{X}\right), \operatorname{Res}\left(\nabla, \mathcal{W}_{X}\right)},
$$

where the subscript refers to the fact that we are taking coinvariants of the above two endomorphisms acting on $\mathcal{V}_{x}$ and $\mathcal{W}_{x}$.

The first map is the composition

$$
\begin{aligned}
& j_{*} j^{*}\left(\mathcal{V}_{X-x} \otimes \mathcal{W}_{X-x} \nabla \mathcal{M}\right) \stackrel{\operatorname{id}_{\mathcal{V}_{X-x}} \otimes_{c a n_{\mathcal{W}}}^{\longrightarrow}}{\longrightarrow} \\
& \rightarrow \Delta_{x_{2}=x_{3} !}\left(j_{*} j^{*}\left(\mathcal{V}_{X-x} \otimes\left(\mathcal{W}_{x} \underset{\mathcal{B}_{x}}{\otimes} \mathcal{M}\right) \operatorname{Res}\left(\nabla, \mathcal{W}_{X}\right)\right)\right) \stackrel{\text { canv }}{\longrightarrow} \\
& \rightarrow \Delta_{!}\left(\mathcal{V}_{x} \underset{\mathcal{B}_{x}}{\otimes} \mathcal{W}_{x} \underset{\mathcal{B}_{x}}{\otimes} \mathcal{M}\right)_{\operatorname{Res}\left(\nabla, \mathcal{V}_{X}\right), \operatorname{Res}\left(\nabla, \mathcal{W}_{X}\right)} .
\end{aligned}
$$


The second map is obtained by interchanging the roles of $\mathcal{V}$ and $\mathcal{W}$. The third map if the composition

$$
\begin{aligned}
& j_{*} j^{*}\left(\mathcal{V}_{X-x} \otimes \mathcal{W}_{X-x} \otimes \mathcal{M}_{x}\right) \rightarrow \Delta_{x_{1}=x_{2} !}\left(j_{*} j^{*}\left(\left(\mathcal{V}_{X-x} \underset{\mathcal{B}_{X-x}}{\otimes} \mathcal{W}_{X-x}\right) \otimes \mathcal{M}_{x}\right)\right) \stackrel{\text { canv } \otimes \mathcal{W}}{\longrightarrow} \\
& \rightarrow \Delta_{!}\left(\mathcal{V}_{x} \underset{\mathcal{B}_{x}}{\otimes} \mathcal{W}_{x} \underset{\mathcal{B}_{x}}{\otimes} \mathcal{M}\right)_{\operatorname{Res}\left(\nabla, \mathcal{V}_{X}\right)+\operatorname{Res}\left(\nabla, \mathcal{W}_{X}\right)} \rightarrow \\
& \rightarrow \Delta_{!}\left(\mathcal{V}_{x} \underset{\mathcal{B}_{x}}{\otimes} \mathcal{W}_{x} \underset{\mathcal{B}_{x}}{\otimes} \mathcal{M}\right)_{\operatorname{Res}\left(\nabla, \mathcal{V}_{X}\right), \operatorname{Res}\left(\nabla, \mathcal{W}_{X}\right)} \cdot
\end{aligned}
$$

Lemma 3.5. The sum of the three maps above is equal to zero.

The proof follows by unfolding the construction of the bijection in the proof of Proposition 3.4.

3.6. End of proof of Theorem 3.2. Consider the following general set-up. Let $\mathcal{A}$ be a chiral algebra, and let $\mathfrak{z}$ be its center. Let $\mathcal{V}$ be a ${ }^{*}$-commutative chiral module over $\mathfrak{z}$, free of finite rank as a $\mathfrak{z}$-module. Let $\mathcal{M}, \mathcal{N}$ be two chiral $\mathcal{A}$-modules.

Given a chiral pairing $\{\mathcal{V} \underset{\mathfrak{z}}{\otimes} \mathcal{A}, \mathcal{M}\} \rightarrow \mathcal{N}$ we can restrict it and obtain a chiral pairing

$$
j_{*} j^{*}(\mathcal{V} \otimes \mathcal{M}) \rightarrow \Delta_{!}(\mathcal{N})
$$

of chiral $\mathfrak{z}$-modules. This map has the following commutation property with respect to $\mathcal{A}$ : the map

$$
\widetilde{j}_{*} \widetilde{j}^{*}(\mathcal{V} \otimes \mathcal{A} \otimes \mathcal{M}) \rightarrow \Delta_{x_{2}=x_{3} !}\left(j_{*} j^{*}(\mathcal{V} \otimes \mathcal{M})\right) \rightarrow \Delta_{!}(\mathcal{N})
$$

equals

$$
\widetilde{j}_{*} \widetilde{j}^{*}(\mathcal{V} \otimes \mathcal{A} \otimes \mathcal{M}) \rightarrow \Delta_{x_{1}=x_{3} !}\left(j_{*} j^{*}(\mathcal{A} \otimes \mathcal{N})\right) \rightarrow \Delta_{!}(\mathcal{N}),
$$

where $\widetilde{j}$ denotes the embedding of the complement of the union of the divisors $x_{1}=x_{3}$ and $x_{2}=x_{3}$.

We have the following general assertion:

Lemma 3.7. The set of $\mathcal{A}$-chiral pairings $\{\mathcal{V} \otimes \mathcal{A}, \mathcal{N}\} \rightarrow \mathcal{N}$ is in a bijection with the set of $\mathfrak{z}$-chiral pairings $\{\mathcal{V}, \mathcal{M}\} \rightarrow \mathcal{N}$, satisfying the additional condition above.

Together with Proposition 3.4, this lemma implies Theorem 3.2.

3.8. Proof of Theorem 1.10. According to Sect. 1.12, in order to prove Theorem 1.10, it suffices to consider the universal case, namely, the one when $\mathcal{M}=$ $\mathfrak{D}_{G, \text { crit }, x}$. We will work in a more general framework, assuming only that $\mathcal{M}$ is such that $\mathcal{F}_{V^{\prime}} \star \mathcal{M}$ is acyclic away from cohomological degree 0 for any $V^{\prime} \in \operatorname{Rep}(\check{G})$, 
which is satisfied in the above case by Theorem 1.13. (Of course, Theorem 1.10 will imply that this assumption is satisfied for any $\mathcal{M}$.)

Composing the map (30) with the tautological projection

we obtain a map

$$
\nu_{\widetilde{\mathfrak{Z}}_{\mathfrak{g}}^{\text {int,reg }}} \underset{\widetilde{\mathfrak{Z}}_{\mathfrak{g}}^{\text {int,reg }}}{\otimes} \mathcal{M} \rightarrow\left(\mathcal{V}_{\widetilde{\mathfrak{Z}}_{\mathfrak{g}}^{\text {int,reg }}} \underset{\widetilde{\mathfrak{Z}}_{\mathfrak{g}}^{\text {int,reg }}}{\otimes} \mathcal{M}\right)_{N_{\widetilde{\mathfrak{J}}_{\mathfrak{g}}^{\text {int,reg }}}},
$$

$$
\mathcal{V}_{\widetilde{\mathfrak{Z}}_{\mathfrak{g}}^{\text {int,reg }}} \underset{\widetilde{\mathfrak{Z}}_{\mathfrak{g}}^{\text {int,reg }}}{\otimes} \mathcal{M} \rightarrow \mathcal{F}_{V} \star \mathcal{M}
$$

We will denote this map by $\mathfrak{s}_{V}^{-1}$, as it will be the inverse of the desired map of Theorem 1.10. We will deduce Theorem 1.10 from the following:

Proposition 3.9. The map of (35) is compatible with tensor products of representations, in the sense that for $V, W \in \operatorname{Rep}(\check{G})$ the 3 compositions

$$
\mathcal{V}_{\widetilde{\mathfrak{Z}}_{\mathfrak{g}}^{\text {int,reg }}} \underset{\widetilde{\mathfrak{Z}}_{\mathfrak{g}}^{\text {int,reg }}}{\otimes} \mathcal{W}_{\widetilde{\mathfrak{Z}}_{\mathfrak{g}}^{\text {int,reg }}} \underset{\widetilde{\mathfrak{Z}}_{\mathfrak{g}}^{\text {int,reg }}}{\otimes} \mathcal{M} \stackrel{\mathfrak{s}_{V}^{-1}}{\longrightarrow} \mathcal{F}_{V} \star\left(\mathcal{W}_{\widetilde{\mathfrak{Z}}_{\mathfrak{g}}^{\text {int,reg }}} \underset{\widetilde{\mathfrak{J}}_{\mathfrak{g}}^{\text {int,reg }}}{\otimes} \mathcal{M}\right) \stackrel{\text { id }_{\mathcal{F}} V^{\star \mathfrak{s}_{W}^{-1}}}{\longrightarrow} \mathcal{F}_{V} \star\left(\mathcal{F}_{W} \star \mathcal{M}\right),
$$

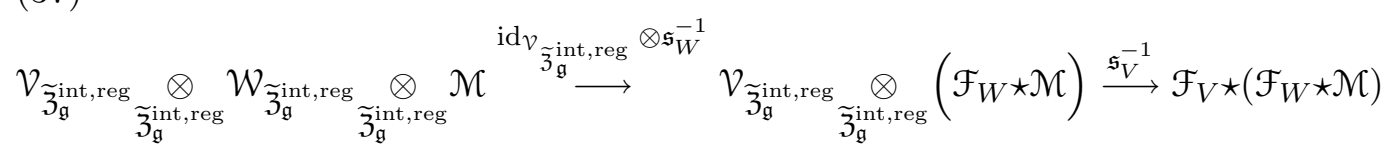

and

$$
\mathcal{V}_{\widetilde{\mathfrak{Z}}_{\mathfrak{g}}^{\text {int,reg }}} \underset{\widetilde{\mathfrak{Z}}_{\mathfrak{g}}^{\text {int,reg }}}{\otimes} \mathcal{W}_{\widetilde{\mathfrak{Z}}_{\mathfrak{g}}^{\text {int,reg }}} \underset{\widetilde{\mathfrak{Z}}_{\mathfrak{g}}^{\text {int,reg }}}{\otimes} \mathcal{M} \stackrel{\mathfrak{s}_{V \otimes W}^{-1}}{\rightarrow} \mathcal{F}_{V \otimes W} \star \mathcal{M} \simeq \mathcal{F}_{V} \star\left(\mathcal{F}_{W} \star \mathcal{M}\right)
$$

coincide.

Let us finish the proof of Theorem 1.10 modulo this proposition. Without restriction of generality, we can assume that the representation $V$ is finitedimensional.

Suppose the map of (35) is not injective, and let $\mathcal{N}$ be its kernel. Let $V^{*}$ be representation dual to $V$. Consider the diagram

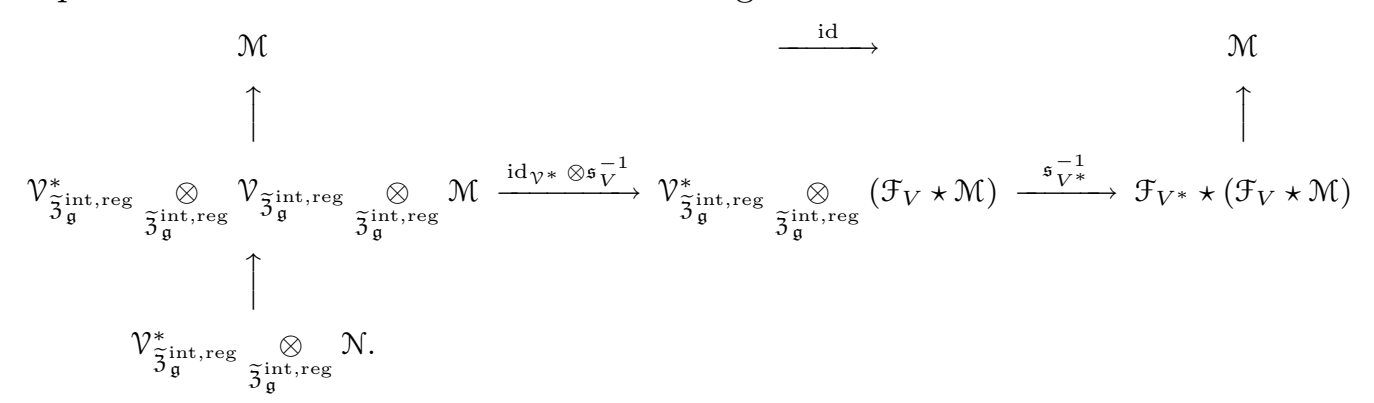


It is commutative since the maps (37) and (38) of Proposition 3.9 coincide. Hence, we obtain that the composed map

$$
\mathcal{V}_{\widetilde{\mathfrak{Z}}_{\mathfrak{g}}^{\text {int,reg }}}^{*} \underset{\widetilde{\mathfrak{Z}}_{\mathfrak{g}}^{\text {int,reg }}}{\otimes} \mathcal{N} \rightarrow \mathcal{M}
$$

is zero. But this is a contradiction since this map is adjoint to the tautological embedding $\mathcal{N} \rightarrow \mathcal{V}_{\widetilde{\mathfrak{Z}}_{\mathfrak{g}}^{\text {int,reg }}} \underset{\widetilde{\mathfrak{Z}}_{\mathfrak{g}}^{\text {int,reg }}}{\otimes} \mathcal{M}$.

In particular, we obtain that $\mathcal{V}_{\widetilde{\mathfrak{Z}}_{\mathfrak{g}}^{\text {int,reg }}} \underset{\widetilde{\mathfrak{Z}}_{\mathfrak{g}}^{\text {int,reg }}}{\otimes} \mathcal{M} \rightarrow\left(\mathcal{V}_{\widetilde{\mathfrak{Z}}_{\mathfrak{g}}^{\text {int,reg }}} \underset{\widetilde{\mathfrak{Z}}_{\mathfrak{g}}^{\text {int,reg }}}{\otimes} \mathcal{M}\right)_{N_{\mathcal{\mathcal { J }}_{\mathfrak{g}}^{\text {int,reg }}}}$ is injective, implying that the action of $N_{\mathcal{V}_{\mathfrak{\mathfrak { g }}}^{\text {int,reg }}}$ on $\mathcal{V}_{\widetilde{\mathfrak{Z}}_{\mathfrak{g}}^{\text {int,reg }}} \underset{\widetilde{\mathfrak{Z}}_{\mathfrak{g}}^{\text {int,reg }}}{\otimes} \mathcal{M}$ is trivial.

Suppose now that the map of (35) is not surjective, and let $\mathcal{N}^{\prime}$ be its kernel. Let us recall that the functors $\mathcal{F}_{V^{\star}}$ ? and $\mathcal{F}_{V^{*}}$ ? are mutually (both left and right) adjoint on the category $D\left(\widehat{\mathfrak{g}}_{\text {crit }}-\bmod \right)^{G[[t]]}$. Consider the diagram

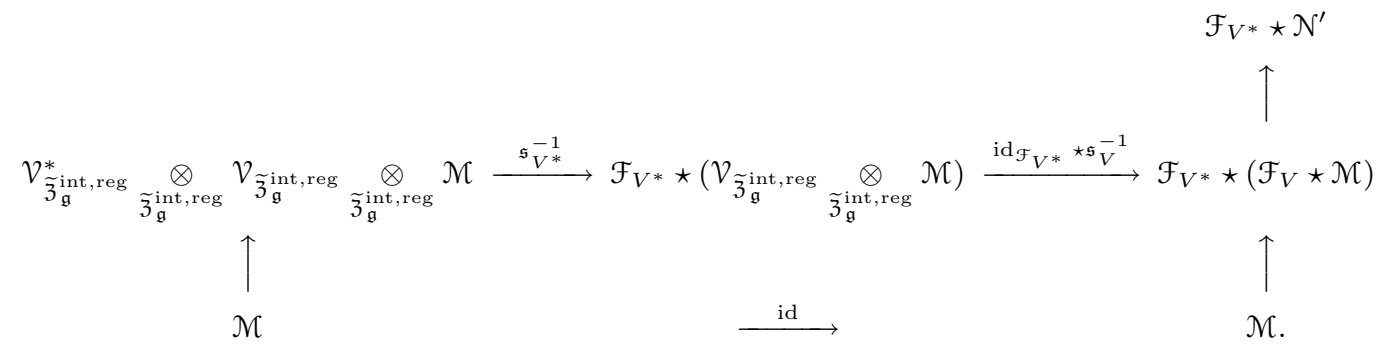

This diagram is commutative because the maps (36) and (38) of Proposition 3.9 coincide. Hence, we obtain that the composed map $\mathcal{M} \rightarrow \mathcal{F}_{V^{*}} \star \mathcal{N}^{\prime}$ is zero, which is a contradiction.

3.10. Proof of Proposition 3.9. Consider the diagram

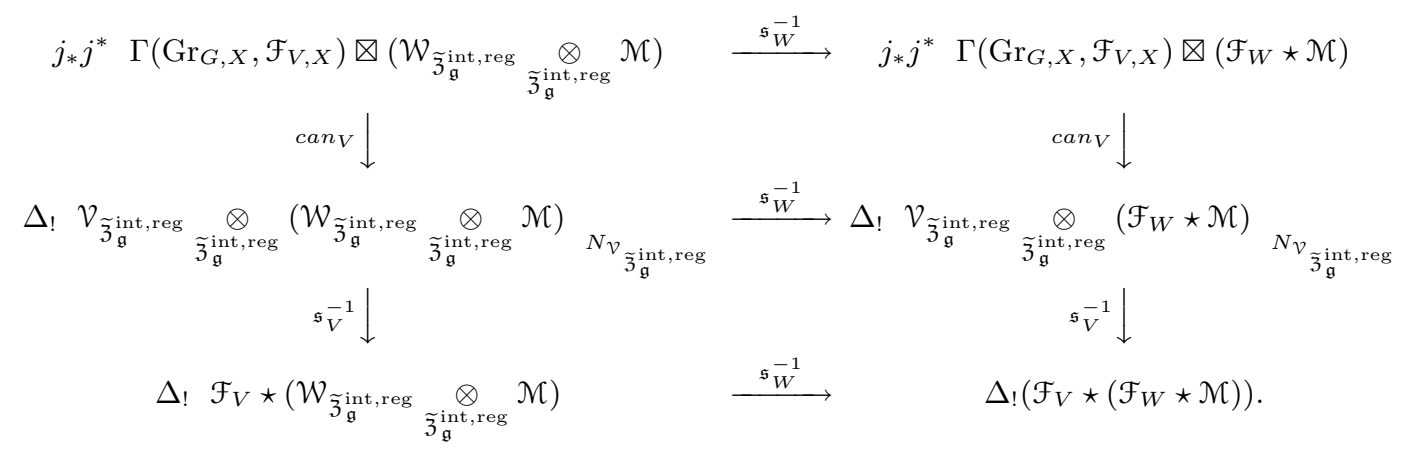

To prove that (36) and (37) coincide we have to show the commutativity of the lower square in this diagram. 
Note that the upper square is commutative by functoriality of the map $c a n_{V}$; moreover the vertical maps in this square are surjective, by Proposition 3.4. Hence, it suffices to see that the outer square is commutative, but this follows from the functoriality of the map (22).

Consider now the composed map

$$
\begin{aligned}
& j_{*} j^{*}\left(\Gamma\left(\operatorname{Gr}_{G, X}, \mathcal{F}_{V, X}\right) \otimes \Gamma\left(\operatorname{Gr}_{G, X}, \mathcal{F}_{W, X}\right) \otimes \mathcal{M}\right) \\
& \downarrow
\end{aligned}
$$

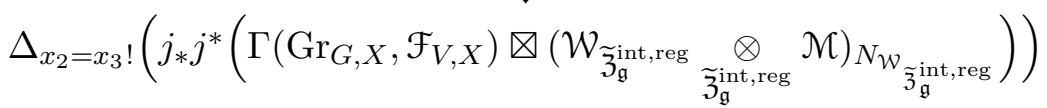

$$
\begin{aligned}
& \downarrow \\
& \Delta_{!}\left(\mathcal{V}_{\widetilde{\mathfrak{Z}}_{\mathfrak{g}}^{\text {int,reg }}} \underset{\widetilde{\mathfrak{Z}}_{\mathfrak{g}}^{\text {int,reg }}}{\otimes} \mathcal{W}_{\widetilde{\mathfrak{Z}}_{\mathfrak{g}}^{\text {int,reg }}} \underset{\widetilde{\mathfrak{J}}_{\mathfrak{g}}^{\text {int,reg }}}{\otimes} \mathcal{M}\right)_{N_{\widetilde{\mathfrak{J}}_{\mathfrak{g}}^{\text {int,reg }}}, N_{\mathcal{W}_{\widetilde{\mathfrak{J}}} \text { int,reg }}} \\
& \text { (36) } \\
& \Delta_{!}\left(\mathcal{F}_{V} \star \mathcal{F}_{W} \star \mathcal{M}\right),
\end{aligned}
$$

and a similar composition when the roles of $V$ and $W$ are interchanged. The resulting maps are equal to the first and the second maps, respectively, of Proposition 2.8 .

Hence, their sum equals the composed map from the commutative diagram

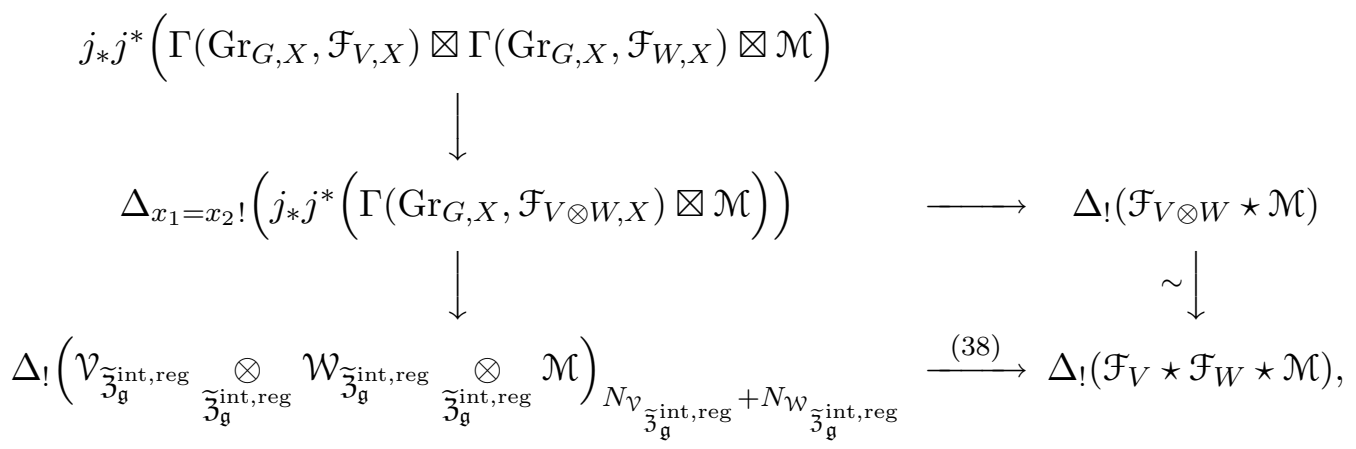

by Proposition 2.8. Moreover, by Lemma 3.5, the sum of the three composed maps

$$
\begin{aligned}
& j_{*} j^{*}\left(\Gamma\left(\operatorname{Gr}_{G, X}, \mathcal{F}_{V, X}\right) \otimes \Gamma\left(\operatorname{Gr}_{G, X}, \mathcal{F}_{W, X}\right) \otimes \mathcal{M}\right) \rightarrow \\
& \rightarrow \Delta_{!}\left(\mathcal{V}_{\widetilde{\mathfrak{Z}}_{\mathfrak{g}}^{\text {int,reg }}} \underset{\widetilde{\mathfrak{J}}_{\mathfrak{g}}^{\text {int,reg }}}{\otimes} \mathcal{W}_{\widetilde{\mathfrak{Z}}_{\mathfrak{g}}^{\text {int,reg }}} \underset{\widetilde{\mathfrak{Z}}_{\mathfrak{g}}^{\text {int,reg }}}{\otimes} \mathcal{M}\right)_{N_{\widetilde{\mathfrak{J}}_{\mathfrak{g}}}}
\end{aligned}
$$

is also zero. 
Let $\mathcal{M}_{V, W} \subset j_{*} j^{*}\left(\Gamma\left(\mathrm{Gr}_{G, X}, \mathcal{F}_{V, X}\right) \otimes \Gamma\left(\mathrm{Gr}_{G, X}, \mathcal{F}_{W, X}\right) \otimes \mathcal{M}\right)$ be the kernel of the map to

$$
\Delta_{x_{1}=x_{3} !}\left(j_{*} j^{*}\left(\Gamma\left(\operatorname{Gr}_{G, X}, \mathcal{F}_{W, X}\right) \otimes\left(\mathcal{V}_{\widetilde{\mathfrak{Z}}_{\mathfrak{g}}^{\text {int,reg }}} \underset{\widetilde{\mathfrak{J}}_{\mathfrak{g}}^{\text {int,reg }}}{\otimes} \mathcal{M}\right)_{N_{\widetilde{\mathcal{J}}_{\mathfrak{g}}^{\text {int,reg }}}}\right)\right) .
$$

Then the map

$$
\mathcal{M}_{V, W} \rightarrow \Delta_{x_{2}=x_{3} !}\left(j_{*} j^{*}\left(\Gamma\left(\operatorname{Gr}_{G, X}, \mathcal{F}_{V, X}\right) \otimes\left(\mathcal{W}_{\widetilde{\mathfrak{Z}_{\mathfrak{g}}}} \underset{\widetilde{\mathfrak{Z}}_{\mathfrak{g}}^{\text {int,reg }}}{\otimes} \mathcal{M}\right)_{N_{\mathcal{W}_{\widetilde{\mathfrak{Z}}} \text { int,reg }}}\right)\right)
$$

is still surjective. Hence, the two surviving maps

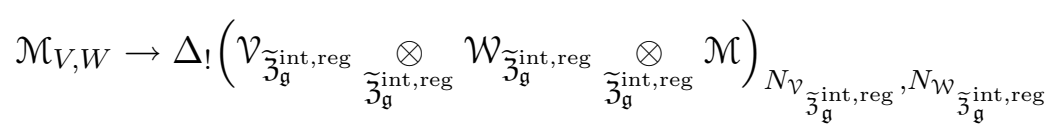

coincide and are surjective. By the above, the two surviving maps

$$
\mathcal{M}_{V, W} \rightarrow \Delta_{!}\left(\mathcal{F}_{V} \star \mathcal{F}_{W} \star \mathcal{M}\right)
$$

coincide as well, and the equality of the maps (36) and (38) follows.

\section{Convolution and fusion for general Chiral Algebras}

The goal of this section is to prove Theorem 2.6. In fact, we will prove a more general result, valid for any chiral algebra, endowed with a Harish-Chandra action of $\operatorname{Jets}(G)_{X}$. We will make a more extensive use of the formalism developed in the appendix to [FG2], but we should note that the main results of this paper, Theorem 1.10 and Theorem 5.4, are independent of this section.

4.1. Twisting of chiral modules. Recall the Lie-* algebras $L_{\mathfrak{g}}$ and $L_{\mathfrak{g}, \kappa}$ introduced in Sect. 1.1. Let $\operatorname{Jets}(G)_{X}$ denote the group-like object in the category of D-schemes on $X$ corresponding to jets into $G$. Its relative cotangent sheaf is the D-module on $X$ canonically isomorphic to the dual $L_{\mathfrak{g}}^{\vee}$ of $L_{\mathfrak{g}}$.

Let $\mathcal{A}$ be a chiral algebra on $X$, endowed with an action of $\operatorname{Jets}(G)_{X}$, such that the adjoint action of its Lie algebra is inner at the level $\kappa$. In other words, we assume being given a homomorphism of Lie-* algebras $L_{\mathfrak{g}, \kappa} \rightarrow \mathcal{A}$, such that the map

$$
\mathcal{A} \rightarrow \mathcal{A} \otimes L_{\mathfrak{g}}^{\vee},
$$

corresponding to the Lie-* bracket equals the derivative of the action of $\operatorname{Jets}(G)$ on $\mathcal{A}$.

Proposition-Construction 4.2. The category of chiral $\mathcal{A}$-modules, supported at $x \in X$ carries an action of the group-scheme $G((t))$ of Harish-Chandra type with respect to the central extension $\widehat{\mathfrak{g}}_{\kappa}$ (cf. [FG2], Sect. 22). 
We will give two proofs. One, discussed below, is local in terms of the De Rham cohomology of $\mathcal{A}$ on the formal punctured disc $\mathcal{D}_{x}^{\times}$around $x$. Another proof will be of chiral nature.

Proof. Let us first recall the following general construction, cf. [CHA], 3.6.9. For a chiral algebra $\mathcal{A}$ let us consider the topological vector space $H_{D R}^{0}\left(\mathcal{D}_{x}^{\times}, \mathcal{A}\right)$. We remind that for a D-module $\mathcal{V}$ on $X$, we define

$$
H_{D R}^{0}\left(\mathcal{D}_{x}^{\times}, \mathcal{V}\right):=\underset{\mathcal{L}}{\lim _{D R}} H_{D R}^{0}\left(\mathcal{D}_{x}^{\times}, \mathcal{L}\right),
$$

where $\mathcal{L}$ runs over the filtered set of finitely-generated D-submodules of $\mathcal{V}$, and the direct limit is taken in the category of topological vector spaces. ${ }^{3}$ Consider in addition the topological vector space $H_{D R}^{0}\left(\mathcal{D}_{x}^{\times} \times \mathcal{D}_{x}^{\times}-\Delta_{\mathcal{D}_{x}^{\times}}, \mathcal{A} \otimes \mathcal{A}\right)$, which is acted on by the transposition $\sigma$. We have a canonical map

$$
H_{D R}^{0}\left(\mathcal{D}_{x}^{\times} \times \mathcal{D}_{x}^{\times}-\Delta_{\mathcal{D}_{x}^{\times}}, \mathcal{A} \otimes \mathcal{A}\right) \rightarrow H_{D R}^{0}\left(\mathcal{D}_{x}^{\times}, \mathcal{A}\right) \vec{\otimes} H_{D R}^{0}\left(\mathcal{D}_{x}^{\times}, \mathcal{A}\right),
$$

defined in fact for any pair of D-modules on $X$, cf. [CHA] 3.6.9.

In addition, the structure of chiral algebra defines a map

$$
\{\cdot, \cdot\}: H_{D R}^{0}\left(\mathcal{D}_{x}^{\times} \times \mathcal{D}_{x}^{\times}-\Delta_{\mathcal{D}_{x}^{\times}}, \mathcal{A} \otimes \mathcal{A}\right) \rightarrow H_{D R}^{0}\left(\mathcal{D}_{x}^{\times}, \mathcal{A}\right) .
$$

The category $\mathcal{A}$-mod ${ }_{x}$ of chiral $\mathcal{A}$-modules supported at $x$ identifies with the category of vector spaces $\mathcal{M}$, endowed with an action map

$$
\operatorname{act}_{\mathcal{M}}: H_{D R}^{0}\left(\mathcal{D}_{x}^{\times}, \mathcal{A}\right) \vec{\otimes} \mathcal{M} \rightarrow \mathcal{M}
$$

such that the difference of

$$
\begin{aligned}
& H_{D R}^{0}\left(\mathcal{D}_{x}^{\times} \times \mathcal{D}_{x}^{\times}-\Delta_{\mathcal{D}_{x}^{\times}}, \mathcal{A} \otimes \mathcal{A}\right) \vec{\otimes} \mathcal{M} \rightarrow H_{D R}^{0}\left(\mathcal{D}_{x}^{\times}, \mathcal{A}\right) \vec{\otimes} H_{D R}^{0}\left(\mathcal{D}_{x}^{\times}, \mathcal{A}\right) \vec{\otimes} \mathcal{M} \stackrel{\mathrm{act}_{\mathcal{M}}}{\longrightarrow} \\
& \rightarrow H_{D R}^{0}\left(\mathcal{D}_{x}^{\times}, \mathcal{A}\right) \vec{\otimes} \mathcal{M} \stackrel{\text { act } \stackrel{\mathcal{M}}{\longrightarrow} \mathcal{M}}{ }
\end{aligned}
$$

and the map obtained by first acting by $\sigma$ on the first factor, equals

$$
H_{D R}^{0}\left(\mathcal{D}_{x}^{\times} \times \mathcal{D}_{x}^{\times}-\Delta_{\mathcal{D}_{x}^{\times}}, \mathcal{A} \otimes \mathcal{A}\right) \vec{\otimes} \mathcal{M} \stackrel{\{\cdot, \cdot\} \otimes i d}{\longrightarrow} H_{D R}^{0}\left(\mathcal{D}_{x}^{\times}, \mathcal{A}\right) \otimes \overrightarrow{\mathcal{M}} \stackrel{\text { act }_{\mathcal{M}}}{\longrightarrow} \mathcal{M} .
$$

Let $\mathbf{g}$ be an $S$-point of $G((t))$ for some base-scheme $S$. It gives rise to a map

$$
\mathcal{O}_{\mathrm{Jets}(G)_{X}} \rightarrow \mathcal{O}_{S}((t))
$$

compatible with the connection. Composing it with the map $\mathcal{A} \rightarrow \mathcal{O}_{\operatorname{Jets}(G)_{X}} \underset{\mathcal{O}_{X}}{\otimes} \mathcal{A}$, given by the action of $\operatorname{Jets}(G)_{X}$ on $\mathcal{A}$, we obtain a map

$$
H_{D R}^{0}\left(\mathcal{D}_{x}^{\times}, \mathcal{A}\right) \rightarrow \mathcal{O}_{S} \stackrel{!}{\otimes} H_{D R}^{0}\left(\mathcal{D}_{x}^{\times}, \mathcal{A}\right) .
$$

\footnotetext{
${ }^{3}$ In other words, we first take the limit in the category of vector spaces, and then complete it in the natural topology.
} 
Similarly, we have a map

$$
H_{D R}^{0}\left(\mathcal{D}_{x}^{\times} \times \mathcal{D}_{x}^{\times}-\Delta_{\mathcal{D}_{x}^{\times}}, \mathcal{A} \otimes \mathcal{A}\right) \rightarrow \mathcal{O}_{S} \stackrel{!}{\otimes} H_{D R}^{0}\left(\mathcal{D}_{x}^{\times} \times \mathcal{D}_{x}^{\times}-\Delta_{\mathcal{D}_{x}^{\times}}, \mathcal{A} \otimes \mathcal{A}\right) .
$$

Given an object $\mathcal{M} \in \mathcal{A}$ - $\bmod _{x}$, we define an action map

$$
H_{D R}^{0}\left(\mathcal{D}_{x}^{\times}, \mathcal{A}\right) \vec{\otimes}\left(\mathcal{M} \otimes \mathcal{O}_{S}\right) \rightarrow \mathcal{M} \otimes \mathcal{O}_{S}
$$

as a composition

$$
\begin{aligned}
& H_{D R}^{0}\left(\mathcal{D}_{x}^{\times}, \mathcal{A}\right) \vec{\otimes}\left(\mathcal{M} \otimes \mathcal{O}_{S}\right) \rightarrow\left(\mathcal{O}_{S} \stackrel{!}{\otimes} H_{D R}^{0}\left(\mathcal{D}_{x}^{\times}, \mathcal{A}\right)\right) \vec{\otimes}\left(\mathcal{M} \otimes \mathcal{O}_{S}\right) \rightarrow \\
& \rightarrow \mathcal{O}_{S} \stackrel{!}{\otimes}\left(H_{D R}^{0}\left(\mathcal{D}_{x}^{\times}, \mathcal{A}\right) \vec{\otimes} \mathcal{M}\right) \stackrel{!}{\otimes} \mathcal{O}_{S} \stackrel{\text { act }^{\prime}}{\rightarrow} \mathcal{O}_{S} \otimes \mathcal{M} \otimes \mathcal{O}_{S} \rightarrow \mathcal{M} \otimes \mathcal{O}_{S},
\end{aligned}
$$

where the last arrow is given by the multiplication on $\mathcal{O}_{S}$.

By construction, it follows that the relation, that singles out representations among all vector spaces endowed with an action of $H_{D R}^{0}\left(\mathcal{D}_{x}^{\times}, \mathcal{A}\right)$ (cf. above), holds. Thus, we obtain a $G((t))$-action on $\mathcal{A}$ - $\bmod { }_{x}$, which is of Harish-Chandra type by construction.

4.3. Twisting and fusion. Let now $\mathcal{M}^{\prime}$ be a torsion-free chiral $\mathcal{A}$-module on $X$. Assume that $\mathcal{M}^{\prime}$ is weakly $\operatorname{Jets}(G)_{X}$-equivariant. I.e., we have an action of $\operatorname{Jets}(G)_{X}$ on $\mathcal{M}^{\prime}$, compatible with its action on $\mathcal{A}$ in the natural sense.

Let $\mathcal{M}, \mathcal{N}$ be two chiral $\mathcal{A}$-modules, both supported at the point $x \in X$. Let $\mathbf{g}$ be an $S$-point of $G((t))$ and let $\mathcal{M}^{\mathrm{g}}$ and $\mathcal{N}^{\mathrm{g}}$ the corresponding $S$-families of objects of $\mathcal{A}$-mod $x$, defined by Proposition-Construction 4.2.

Proposition-Construction 4.4. To every chiral pairing

$$
\phi: j_{*} j^{*}\left(\mathcal{M}^{\prime} \otimes \mathcal{M}\right) \rightarrow \Delta_{!}(\mathcal{N})
$$

there functorially corresponds a chiral pairing

$$
\phi: j_{*} j^{*}\left(\mathcal{M}^{\prime} \otimes \mathcal{M}^{\mathbf{g}}\right) \rightarrow \Delta_{!}\left(\mathcal{N}^{\mathbf{g}}\right) .
$$

Proof. The proof is largely parallel to that of Proposition-Construction 4.2 above.

First, let $\mathcal{M}^{\prime}$ be any torsion-free module over a chiral algebra $\mathcal{A}$. Consider the topological vector spaces $H_{D R}^{0}\left(\mathcal{D}_{x}^{\times}, \mathcal{M}^{\prime}\right)$ and $H_{D R}^{0}\left(\mathcal{D}_{x}^{\times} \times \mathcal{D}_{x}^{\times}-\Delta_{\mathcal{D}_{x}^{\times}}, \mathcal{A} \otimes \mathcal{M}^{\prime}\right)$; the action of $\mathcal{A}$ on $\mathcal{M}^{\prime}$ gives rise to a map

$$
\operatorname{act}_{\mathcal{M}^{\prime}}: H_{D R}^{0}\left(\mathcal{D}_{x}^{\times} \times \mathcal{D}_{x}^{\times}-\Delta_{\mathcal{D}_{x}^{\times}}, \mathcal{A} \otimes \mathcal{M}^{\prime}\right) \rightarrow H_{D R}^{0}\left(\mathcal{D}_{x}^{\times}, \mathcal{M}^{\prime}\right) \text {. }
$$

For two objects $\mathcal{M}, \mathcal{N} \in \mathcal{A}$-mod ${ }_{x}$, chiral pairings $\left\{\mathcal{N}^{\prime}, \mathcal{N}\right\} \rightarrow \mathcal{N}$ are in bijection with maps

$$
\phi: H_{D R}^{0}\left(\mathcal{D}_{x}^{\times}, \mathcal{M}^{\prime}\right) \vec{\otimes} \mathcal{M} \rightarrow \mathcal{N}
$$


such that two difference of the two compositions

$$
\begin{aligned}
& H_{D R}^{0}\left(\mathcal{D}_{x}^{\times} \times \mathcal{D}_{x}^{\times}-\Delta_{\mathcal{D}_{x}^{\times}}, \mathcal{A} \otimes \mathcal{M}^{\prime}\right) \vec{\otimes} \mathcal{M} \rightarrow H_{D R}^{0}\left(\mathcal{D}_{x}^{\times}, \mathcal{A}\right) \vec{\otimes} H_{D R}^{0}\left(\mathcal{D}_{x}^{\times}, \mathcal{M}^{\prime}\right) \vec{\otimes} \mathcal{M} \stackrel{\text { id } \mathcal{A} \otimes \phi}{\longrightarrow} \\
& \rightarrow H_{D R}^{0}\left(\mathcal{D}_{x}^{\times}, \mathcal{A}\right) \vec{\otimes} \mathcal{N} \stackrel{\text { act, }_{\mathcal{N}}}{\longrightarrow} \mathcal{N}
\end{aligned}
$$

and

$H_{D R}^{0}\left(\mathcal{D}_{x}^{\times} \times \mathcal{D}_{x}^{\times}-\Delta_{\mathcal{D}_{x}^{\times}}, \mathcal{A} \otimes \mathcal{M}^{\prime}\right) \vec{\otimes} \mathcal{M} \rightarrow H_{D R}^{0}\left(\mathcal{D}_{x}^{\times}, \mathcal{M}^{\prime}\right) \vec{\otimes} H_{D R}^{0}\left(\mathcal{D}_{x}^{\times}, \mathcal{A}\right) \vec{\otimes} \mathcal{M} \stackrel{\text { id }_{\mathcal{M}^{\prime}} \otimes \operatorname{act}_{\mathcal{M}}}{\longrightarrow}$

$\rightarrow H_{D R}^{0}\left(\mathcal{D}_{x}^{\times}, \mathcal{M}^{\prime}\right) \vec{\otimes} \mathcal{M} \stackrel{\phi}{\rightarrow} \mathcal{N}$

equals

$$
H_{D R}^{0}\left(\mathcal{D}_{x}^{\times} \times \mathcal{D}_{x}^{\times}-\Delta_{\mathcal{D}_{x}^{\times}}, \mathcal{A} \otimes \mathcal{M}^{\prime}\right) \vec{\otimes} \mathcal{M} \stackrel{\text { act }_{\mathcal{M}^{\prime}}}{\longrightarrow} H_{D R}^{0}\left(\mathcal{D}_{x}^{\times}, \mathcal{M}^{\prime}\right) \vec{\otimes} \mathcal{M} \stackrel{\phi}{\rightarrow} \mathcal{N} .
$$

Suppose now that $\mathcal{M}^{\prime}$ is weakly $\operatorname{Jets}(G)_{X}$-equivariant. Given an $S$-point $\mathbf{g}$ of $G((t))$ as above, as in the case of $\mathcal{A}$, we obtain a map

$$
H_{D R}^{0}\left(\mathcal{D}_{x}^{\times}, \mathcal{M}^{\prime}\right) \rightarrow \mathcal{O}_{S} \stackrel{!}{\otimes} H_{D R}^{0}\left(\mathcal{D}_{x}^{\times}, \mathcal{M}^{\prime}\right) .
$$

For a chiral pairing $\phi$ as above, we define a chiral pairing

$$
H_{D R}^{0}\left(\mathcal{D}_{x}^{\times}, \mathcal{M}^{\prime}\right) \vec{\otimes}\left(\mathcal{M} \otimes \mathcal{O}_{S}\right) \rightarrow\left(\mathcal{N} \otimes \mathcal{O}_{S}\right)
$$

as a composition

$$
\begin{aligned}
& H_{D R}^{0}\left(\mathcal{D}_{x}^{\times}, \mathcal{M}^{\prime}\right) \vec{\otimes}\left(\mathcal{M} \otimes \mathcal{O}_{S}\right) \rightarrow\left(\mathcal{O}_{S} \stackrel{!}{\otimes} H_{D R}^{0}\left(\mathcal{D}_{x}^{\times}, \mathcal{M}^{\prime}\right)\right) \vec{\otimes}\left(\mathcal{M} \otimes \mathcal{O}_{S}\right) \rightarrow \\
& \rightarrow \mathcal{O}_{S} \stackrel{!}{\otimes}\left(H_{D R}^{0}\left(\mathcal{D}_{x}^{\times}, \mathcal{M}^{\prime}\right) \vec{\otimes} \mathcal{M}\right) \stackrel{!}{\otimes} \mathcal{O}_{S} \stackrel{i d \otimes \phi \otimes i d}{\longrightarrow} \mathcal{O}_{S} \otimes \mathcal{N} \otimes \mathcal{O}_{S} \rightarrow \mathcal{N} \otimes \mathcal{O}_{S} .
\end{aligned}
$$

It is easy to see that the relation involving the $\mathcal{A}$-actions on $\mathcal{M}$ and $\mathcal{N}$, described above, holds.

Let us assume now that in the circumstances of the above proposition, module $\mathcal{M}^{\prime}$ is strongly $\operatorname{Jets}(G)_{X}$-equivariant. By definition, this means that the derivative of the group-action, which is a map

$$
\mathcal{M}^{\prime} \rightarrow \mathcal{M}^{\prime} \otimes L_{\mathfrak{g}}^{\vee}
$$

coincides with the one coming from the Lie-* action of $L_{\mathfrak{g}, \kappa}$ via $L_{\mathfrak{g}, \kappa} \rightarrow \mathcal{A}$.

Let $\mathbf{g}_{1}$ and $\mathbf{g}_{2}$ be two $S$-points of $G((t))$, whose ratio is a map from $S$ to the first infinitesimal neighborhood of the identity in $G((t))$. In particular, for every choice of the splitting $\mathfrak{g}((t)) \rightarrow \widehat{\mathfrak{g}}_{\kappa}$ we have the canonical isomorphisms $\mathcal{N}^{\mathbf{g}_{1}} \simeq \mathcal{N}^{\mathbf{g}_{2}}$ and $\mathcal{N}^{g_{1}} \simeq \mathcal{N}^{g_{2}}$.

From the proof of Proposition-Construction 4.4 we obtain: 
Corollary 4.5. Under the above circumstances, the diagram

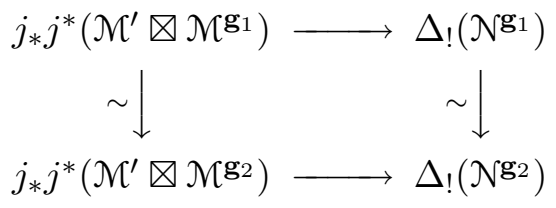

is commutative.

Let now $\mathcal{F}^{\prime}$ be a $\kappa$-twisted D-module on $G((t))$, which strongly $K$-equivariant on the right, where $K$ is an "open-compact" group-subscheme of $G((t))$. By [FG2], Sect. 22.4, given an object $\mathcal{N} \in \mathcal{A}$ - $\bmod _{x}$, which is strongly $K$-equivariant, there exists a well-defined complex of objects in $\mathcal{A}$-mod ${ }_{x}$ :

$$
\mathfrak{C}^{\frac{\infty}{2}}\left(\mathfrak{g}((t)) ; K_{\text {red }}, \mathcal{F}^{\prime} \otimes \mathcal{M}\right),
$$

where $K_{\text {red }}$ denotes the reductive quotient of $K$.

The image of this complex in the derived category is by definition the convolution $\mathcal{F} \star \mathcal{M}$, where $\mathcal{F}$ is the twisted $\mathrm{D}$-module on $G / K$, corresponding to $\mathcal{F}^{\prime}$.

From Proposition-Construction 4.4 above, we obtain that given two strongly $K$-equivariant objects $\mathcal{N}, \mathcal{N} \in \mathcal{A}$-mod ${ }_{x}$ and a chiral pairing $\left\{\mathcal{M}^{\prime}, \mathcal{N}\right\} \rightarrow \mathcal{N}$ we obtain a chiral pairing of graded objects

$$
\left\{\mathcal{M}^{\prime}, \mathfrak{C}^{\frac{\infty}{2}}\left(\mathfrak{g}((t)) ; K_{\text {red }}, \mathcal{F}^{\prime} \otimes \mathcal{M}\right)\right\} \rightarrow \mathfrak{C}^{\frac{\infty}{2}}\left(\mathfrak{g}((t)) ; K_{\text {red }}, \mathcal{F}^{\prime} \otimes \mathcal{N}\right) .
$$

Moreover, by Corollary 4.5, the above pairing is a map of complexes, i.e., it respects the differentials on both sides.

As an application we shall now establish the following result. Let $\operatorname{Av}_{G[[t]]}$ denote the functor $D^{+}\left(\mathcal{A}-\bmod _{x}\right) \rightarrow D^{+}\left(\mathcal{A}-\bmod _{x}\right)^{G[[t]]}$, right adjoint to the forgetful functor. In particular, it is left-exact and the functor

$$
\mathcal{N} \rightarrow h^{0}\left(\operatorname{Av}_{G[[t]]}(\mathcal{N})\right)
$$

is the right adjoint to the forgetful functor $\mathcal{A}-\bmod _{x}^{G[[t]]} \rightarrow \mathcal{A}$-mod ${ }_{x}$.

Proposition 4.6. Let $\left\{\mathcal{N}^{\prime}, \mathcal{M}\right\} \rightarrow \mathcal{N}$ be a chiral pairing with $\mathcal{M}^{\prime}$ being strongly $\operatorname{Jets}(G)_{X}$-equivariant, and $\mathcal{M} \in \mathcal{A}$-mod $\bmod _{x}$ being $G[[t]]$-equivariant. Then this pairing canonically factors through $\left\{\mathcal{N}^{\prime}, \mathcal{M}\right\} \rightarrow h^{0}\left(\operatorname{Av}_{G[[t]]}(\mathcal{N})\right)$.

Proof. Let us recall that the functor $\operatorname{Av}_{G[[t]]}$ is represented by the complex

$$
\mathcal{N} \mapsto \mathfrak{C}^{\bullet}\left(\mathfrak{g}[[t]], \mathcal{O}_{G[[t]]} \otimes \mathcal{N}\right)
$$


Hence, as in (40), given a chiral pairing $\left\{\mathcal{M}^{\prime}, \mathcal{M}\right\} \rightarrow \mathcal{N}$, we obtain a chiral pairing of complexes

$$
\left\{\mathcal{M}^{\prime}, \operatorname{Av}_{G[[t]]}(\mathcal{N})\right\} \rightarrow \operatorname{Av}_{G[[t]]}(\mathcal{N}),
$$

compatible with the differential. Composing with the canonical map $\mathcal{M} \rightarrow$ $\operatorname{Av}_{G[[t]]}(\mathcal{M})$, we obtain the desired chiral pairing

$$
\left\{\mathcal{M}^{\prime}, \mathcal{M}\right\} \rightarrow \operatorname{Av}_{G[[t]]}(\mathcal{N}) \text {. }
$$

4.7. The global case. We shall now generalize the discussion of the previous subsections to the case of chiral $\mathcal{A}$-modules, which are not necessarily supported at a single point $x \in X$.

Recall that in addition to $\operatorname{Jets}(G)_{X}$, we have the group D-ind scheme $\operatorname{Jets}^{\operatorname{mer}}(G)_{X}$.

\section{Proposition-Construction 4.8.}

(1) Let $\mathcal{M}$ be a chiral $\mathcal{A}$-module on $X$, and let $\mathbf{g}$ be an $S$-point of $\operatorname{Jets}^{m e r}(G)$, where $S$ is an affine $D$-scheme on $X$. Then the D-module $\mathcal{M}^{\mathrm{g}}:=\mathcal{M} \underset{\mathcal{O}_{X}}{\otimes} \mathcal{O}_{S}$ acquires a natural structure of chiral $\mathcal{A}$-module.

(2) If the ratio of two points $\mathbf{g}_{1}$ and $\mathbf{g}_{2}$ lies in the first infinitesimal neighborhood of the unit section of Jets ${ }^{\text {mer }}(G)$, then for every choice of the splitting as a Dmodule $L_{\mathfrak{g}} \rightarrow L_{\mathfrak{g}, \kappa}$ there exists a functorial isomorphism $\mathcal{M}^{\mathbf{g}_{1}} \simeq \mathcal{M}^{\mathbf{g}_{2}}$.

(3) If $\mathcal{M}^{\prime}$ is a chiral $\mathcal{A}$-module, which is weakly $\operatorname{Jets}(G)$-equivariant, then to every chiral pairing $\left\{\mathcal{N}^{\prime}, \mathcal{M}\right\} \rightarrow \mathcal{N}$ there functorially corresponds a chiral pairing $\left\{\mathcal{N}^{\prime}, \mathcal{N}^{\mathrm{g}}\right\} \rightarrow \mathcal{N}^{\mathrm{g}}$.

(4) In the circumstances of points (2) and (3) above assume in addition that $\mathcal{M}^{\prime}$ is strongly $\operatorname{Jets}(G)$-equivariant. Then the diagram of Corollary 4.5 commutes.

Proof. Let us first recall the following general construction. Let

$$
j_{*} j^{*}\left(\mathcal{M}_{1}^{i} \otimes \mathcal{M}_{2}^{i}\right) \rightarrow \Delta_{!}\left(\mathcal{N}^{i}\right),
$$

be maps of D-modules, where $i$ runs over some finite set of indices $i$. Then we have a map

$$
j_{*} j^{*}\left(\left(\underset{i}{\otimes \mathcal{M}_{1}^{i}}\right) \otimes \underset{i}{\left.\left(\underset{\mathcal{M}}{\mathcal{M}_{2}^{i}}\right)\right)} \rightarrow \underset{i}{\Delta} \underset{i}{\otimes \mathcal{N}^{i}}\right) .
$$

Let us recall also that the data of an $S$-point of $\operatorname{Jets}^{m e r}(G)$ is equivalent to that of a map

$$
j_{*} j^{*}\left(\mathcal{O}_{\mathrm{Jets}(G)_{X}} \otimes \mathcal{O}_{X}\right) \rightarrow \Delta_{!}\left(\mathcal{O}_{S}\right),
$$


such that the diagram

$$
\begin{array}{ccc}
j_{*} j^{*}\left(\left(\mathcal{O}_{\operatorname{Jets}(G)_{X}} \otimes \mathcal{O}_{\operatorname{Jets}(G)_{X}}\right) \otimes \mathcal{O}_{X}\right) & \longrightarrow & \Delta_{!}\left(\left(\mathcal{O}_{S} \otimes \mathcal{O}_{S}\right) \otimes \mathcal{O}_{X}\right) \\
\downarrow & \downarrow \\
j_{*} j^{*}\left(\mathcal{O}_{\operatorname{Jets}(G)_{X}} \otimes \mathcal{O}_{X}\right) & \longrightarrow & \Delta_{!}\left(\mathcal{O}_{S}\right)
\end{array}
$$

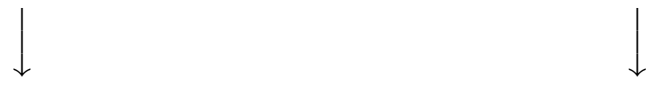

commutes, where the upper horizontal arrow comes from the map (41), and vertical arrows are given by the algebra multiplication.

As in the proofs of Proposition-Construction 4.2 and PropositionConstruction 4.4, the proof follows from the next general construction. Let

$$
j_{*} j^{*}\left(\mathcal{M}_{1} \otimes \mathcal{M}_{2}\right) \rightarrow \Delta_{!}(\mathcal{N}) \text { and } \mathcal{M}_{1} \rightarrow \mathcal{M}_{1} \otimes \mathcal{O}_{\operatorname{Jets}(G)_{X}}
$$

be maps of D-modules and $\mathbf{g}$ be as above. Then from (41) we obtain a map

$$
j_{*} j^{*}\left(\mathcal{M}_{1} \otimes \mathcal{M}_{2}\right) \rightarrow j_{*} j^{*}\left(\left(\mathcal{M}_{1} \otimes \mathcal{O}_{\operatorname{Jets}(G)_{X}}\right) \otimes\left(\mathcal{M}_{2} \otimes \mathcal{O}_{S}\right)\right) \rightarrow \Delta_{!}\left(\mathcal{N} \otimes \mathcal{O}_{S}\right),
$$

which, in turn, gives rise to a map

$$
j_{*} j^{*}\left(\mathcal{M}_{1} \otimes\left(\mathcal{M}_{2} \otimes \mathcal{O}_{S}\right)\right) \rightarrow \Delta_{!}\left(\mathcal{N} \otimes \mathcal{O}_{S}\right) .
$$

By putting first $\mathcal{M}_{1}:=\mathcal{A}, \mathcal{M}_{2}:=\mathcal{M}$ and $\mathcal{N}:=\mathcal{M}$, we arrive to the chiral action map of point (1). By putting $\mathcal{M}_{1}:=\mathcal{M}^{\prime}, \mathcal{M}_{2}:=\mathcal{M}$ and $\mathcal{N}:=\mathcal{N}$, we arrive to the chiral pairing of point (3).

To prove point (2), we can assume being given a map

$$
j_{*} j^{*}\left(L_{\mathfrak{g}}^{\vee} \otimes \mathcal{O}_{X}\right) \rightarrow \Delta_{!}\left(\mathcal{O}_{S}\right),
$$

and we have to construct a map $\varphi: \mathcal{M} \rightarrow \mathcal{M} \otimes \mathcal{O}_{S}$, which fits into the commutative diagram:

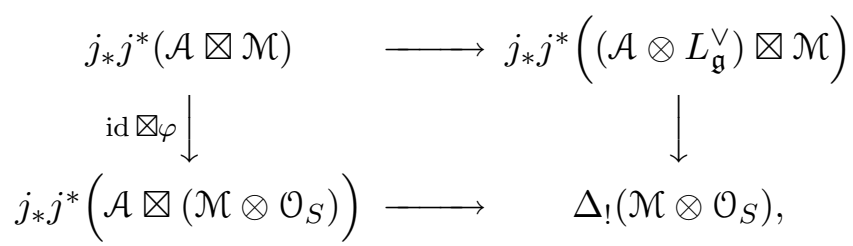

where the right vertical arrow comes from (41), and the bottom horizontal arrow comes from the initial chiral action of $\mathcal{A}$ on $\mathcal{M}$.

The desired map $\varphi$ is constructed as follows. The chiral bracket of $L_{\mathfrak{g}}$ with $\mathcal{M}$ (which is well-defined since we chose a splitting of $L_{\mathfrak{g}, \kappa}$ ) and (41) give rise to a map

$$
j_{*} j^{*}\left(\left(L_{\mathfrak{g}} \otimes L_{\mathfrak{g}}^{\vee}\right) \otimes \mathcal{M}\right) \rightarrow \Delta_{!}\left(\mathcal{M} \otimes \mathcal{O}_{S}\right)
$$

The required map if the Lie-* bracket induced by the above map applied to the canonical element $\mathbf{1} \in H_{D R}^{0}\left(L_{\mathfrak{g}} \otimes L_{\mathfrak{g}}^{\vee}\right)$. 
The fact that the axioms are satisfied, and point (4) of the proposition follow from the construction.

Let $\mathcal{M}$ be a chiral $\mathcal{A}$-module as above, and let $\mathcal{F}^{\prime}$ be a chiral module over $\mathfrak{D}_{G, \kappa}$. Then Proposition-Construction 4.8 implies that proceeding as in [FG2], Sect. 22.4, we can form a twisted product of $\mathcal{F}^{\prime}$ and $\mathcal{M}$, denoted $\mathcal{F}^{\prime} \widetilde{\otimes} \mathcal{M}$.

Let us write down this construction explicitly. As a D-module, this will be the usual tensor product $\mathcal{F}^{\prime} \otimes \mathcal{M}$, but it will carry a new action of $\mathcal{A}$, and a commuting action of $\mathcal{A}_{\mathfrak{g}, 2 \kappa_{\text {crit }}}$. (Note that $2 \kappa_{\text {crit }}$ equals the negative of the Killing form on $\mathfrak{g}$.)

Namely, the action of $\mathcal{A}$ is the composition:

$$
j_{*} j^{*}\left(\mathcal{A} \otimes\left(\mathcal{F}^{\prime} \otimes \mathcal{M}\right)\right) \rightarrow j_{*} j^{*}\left(\left(\mathcal{O}_{\operatorname{Jets}(G)_{X}} \otimes \mathcal{A}\right) \otimes\left(\mathcal{F}^{\prime} \otimes \mathcal{M}\right)\right) \rightarrow \Delta_{!}\left(\mathcal{F}^{\prime} \otimes \mathcal{M}\right),
$$

where the last arrow comes from the chiral action of $\mathcal{O}_{\text {Jets }(G)_{X}}$ on $\mathcal{F}$ and $\mathcal{A}$ on $\mathcal{M}$ via (41).

The chiral action of $L_{\mathfrak{g}, 2 \kappa_{\text {crit }}}$ is the diagonal one with respect to the $\mathfrak{r}$-action of $\mathcal{A}_{\mathfrak{g}, \kappa^{\prime}}$ on $\mathcal{F}^{\prime}$ and the action of $L_{\mathfrak{g}, \kappa}$ on $\mathcal{M}$ that comes from $L_{\mathfrak{g}, \kappa} \rightarrow \mathcal{A}$.

Hence, by tensoring with tensoring with the Clifford chiral algebra, we obtain a well-defined complex $\mathfrak{C}^{\frac{\infty}{2}}\left(L_{\mathfrak{g}}, \mathcal{F}^{\prime} \otimes \mathcal{M}\right)$ of chiral $\mathcal{A}$-modules. More generally, if $\left\{\mathcal{M}^{\prime}, \mathcal{M}\right\} \rightarrow \mathcal{N}$ is a chiral pairing of $\mathcal{A}$-modules, with $\mathcal{M}^{\prime}$ being $\operatorname{Jets}(G)_{X^{-}}$ equivariant, as in point (3) of the above proposition, we obtain a chiral pairing of complexes

$$
j_{*} j^{*}\left(\mathcal{M}^{\prime} \otimes \mathfrak{C}^{\frac{\infty}{2}}\left(L_{\mathfrak{g}}, \mathcal{F}^{\prime} \otimes \mathcal{M}\right)\right) \rightarrow \Delta_{!}\left(\mathfrak{C}^{\frac{\infty}{2}}\left(L_{\mathfrak{g}}, \mathcal{F}^{\prime} \otimes \mathcal{N}\right)\right)
$$

In particular, if $\mathcal{F}^{\prime}$ is strongly equivariant with respect to the right action of $\operatorname{Jets}(G)_{X}$ and $\mathcal{M}$ is also $\operatorname{Jets}(G)_{X}$-equivariant, by considering the corresponding subcomplex of chains relative to $\mathfrak{g} \in \Gamma\left(X, \mathcal{L}_{\mathfrak{g}}\right)$, we obtain a map

$$
j_{*} j^{*}\left(\mathcal{M}^{\prime} \otimes \mathfrak{C}^{\frac{\infty}{2}}\left(L_{\mathfrak{g}} ; \mathfrak{g}, \mathcal{F}^{\prime} \otimes \mathcal{M}\right)\right) \rightarrow \Delta_{!}\left(\mathfrak{C}^{\frac{\infty}{2}}\left(L_{\mathfrak{g}} ; \mathfrak{g}, \mathcal{F}^{\prime} \otimes \mathcal{N}\right)\right),
$$

and on the level of individual cohomologies the chiral pairings

$$
j_{*} j^{*}\left(\mathcal{M}^{\prime} \otimes h^{i}(\mathcal{F} \star \mathcal{M})\right) \rightarrow \Delta_{!}\left(h^{i}(\mathcal{F} \star \mathcal{N})\right),
$$

where $\mathcal{F}$ denotes the corresponding twisted D-module on $\mathrm{Gr}_{G, X}$.

4.9. Some compatibilities. Let us take as an example the case when $\mathcal{M}=\mathcal{A}$, and the canonical chiral pairing $\left\{\mathcal{N}^{\prime}, \mathcal{A}\right\} \rightarrow \mathcal{M}^{\prime}$. We obtain the chiral pairings

$$
\left\{\mathcal{M}^{\prime}, h^{i}(\mathcal{F} \star \mathcal{A})\right\} \rightarrow h^{i}\left(\mathcal{F} \star \mathcal{M}^{\prime}\right) .
$$


Consider the case $\mathcal{A} \simeq \mathcal{A}_{\mathfrak{g}, \kappa}$. Then, by construction,

$$
\mathcal{F} \star \mathcal{A}_{\mathfrak{g}, \kappa} \simeq \Gamma\left(\operatorname{Gr}_{G, X}, \mathcal{F}\right) .
$$

Lemma 4.10. For $\mathcal{A} \simeq \mathcal{A}_{\mathfrak{g}, \kappa}$, the chiral pairings of (45) coincide with those of (18).

Proof. By the construction of the pairings in (45), it is sufficient to consider the case when $\mathcal{M}^{\prime} \simeq \mathfrak{D}_{G, \kappa}$. The latter reduces to the case of the chiral algebra $\mathfrak{D}_{G, \kappa}$ rather than $\mathcal{A}_{\mathfrak{g}, \kappa}$.

Now the assertion of the lemma follows from the next general observation: if $\mathcal{M}^{\prime}=\mathcal{A}$, then the map of Proposition-Construction 4.8(3) coincides with the chiral action of $\mathcal{A}$ on $\mathcal{M}^{\mathrm{g}}$. In particular, the maps of (44) are also given by the chiral action of $\mathcal{A}$ on $h^{i}(\mathcal{F} \star \mathcal{M})$.

Let us now establish some further compatibilities, satisfied by the maps of Proposition-Construction 4.8.

Let $\mathcal{M}_{1}, \mathcal{M}_{2}, \mathcal{M}_{3}$ be chiral modules over $\mathcal{A}$, and let $\left\{\mathcal{M}_{1}, \mathcal{M}_{2}\right\} \rightarrow \mathcal{M}_{1,2}$, $\left\{\mathcal{N}_{2}, \mathcal{M}_{3}\right\} \rightarrow \mathcal{M}_{2,3},\left\{\mathcal{M}_{1}, \mathcal{M}_{3}\right\} \rightarrow \mathcal{M}_{1,3}$ be chiral pairings. In addition, let $\left\{\mathcal{M}_{1,2}, \mathcal{M}_{3}\right\} \rightarrow \mathcal{N},\left\{\mathcal{M}_{2,3}, \mathcal{M}_{1}\right\} \rightarrow \mathcal{N},\left\{\mathcal{N}_{1,3}, \mathcal{N}_{2}\right\} \rightarrow \mathcal{N}$ be chiral pairings such that the sum of the three maps

$$
j_{*} j^{*}\left(\mathcal{M}_{1} \otimes \mathcal{M}_{2} \otimes \mathcal{M}_{3}\right) \rightarrow \Delta_{!}(\mathcal{N})
$$

equals zero.

Assume also that all of the above modules are $\operatorname{Jets}(G)_{X}$-equivariant, and let $\mathcal{F}$ be a twisted D-module on $\mathrm{Gr}_{G, X}$.

Lemma 4.11. Under the above circumstances the sum of the three induced maps

$$
j_{*} j^{*}\left(h^{i}\left(\mathcal{F} \star \mathcal{M}_{1}\right) \otimes \mathcal{M}_{2} \otimes \mathcal{M}_{3}\right) \rightarrow \Delta_{!}\left(h^{i}(\mathcal{F} \star \mathcal{N})\right)
$$

is zero.

Proof. By the construction of convolution, it suffices to note the following. Let $\mathcal{M}_{1}, \mathcal{M}_{2}, \mathcal{M}_{3}, \mathcal{M}_{1,2}, \mathcal{M}_{2,3}, \mathcal{M}_{1,3}, \mathcal{N}$ be as above, but let us only assume that $\mathcal{M}_{2}, \mathcal{M}_{3}$ and $\mathcal{M}_{2,3}$ are $\operatorname{Jets}(G)_{X}$-equivariant. Let $\mathbf{g}$ be an $S$-point of Jets ${ }^{m e r}(G)_{X}$ for some D-scheme $S$.

Then we have the chiral pairings,

$$
\begin{aligned}
& \left\{\mathcal{M}_{1}^{\mathbf{g}}, \mathcal{M}_{2}\right\} \rightarrow \mathcal{M}_{1,2}^{\mathbf{g}},\left\{\mathcal{M}_{1}, \mathcal{M}_{3}\right\} \rightarrow \mathcal{M}_{1,3}^{\mathbf{g}}, \\
& \left\{\mathcal{M}_{1,2}^{\mathbf{g}}, \mathcal{M}_{3}\right\} \rightarrow \mathcal{N}^{\mathbf{g}},\left\{\mathcal{M}_{1,3}^{\mathbf{g}}, \mathcal{M}_{2}\right\} \rightarrow \mathcal{N}^{\mathbf{g}},\left\{\mathcal{M}_{1}^{\mathbf{g}}, \mathcal{M}_{2,3}\right\} \rightarrow \mathcal{N}^{\mathbf{g}},
\end{aligned}
$$


and the sum of the resulting three maps

$$
j_{*} j^{*}\left(\mathcal{M}_{1}^{\mathbf{g}} \otimes \mathcal{M}_{2} \otimes \mathcal{M}_{3}\right) \rightarrow \Delta_{!}\left(\mathcal{N}^{\mathbf{g}}\right)
$$

is zero.

Let now $\mathcal{F}_{1}^{\prime}, \mathcal{F}_{2}^{\prime}$ be two chiral $\mathfrak{D}_{G, \kappa}$-modules with $\mathcal{F}_{1}^{\prime}$ being strongly $\operatorname{Jets}(G)_{X^{-}}$ equivariant on the right and $\mathcal{F}_{2}^{\prime}$ being strongly $\operatorname{Jets}(G)_{X}$-equivariant on the left. Let $\mathcal{F}_{1}^{\prime} \star \mathcal{F}_{2}^{\prime}$ be their convolution, which is by definition represented by the complex

$$
\mathfrak{C}^{\frac{\infty}{2}}\left(L_{\mathfrak{g}} ; \mathfrak{g}, \mathcal{F}_{1}^{\prime} \otimes \mathcal{F}_{2}^{\prime}\right) .
$$

Given a chiral pairing $\left\{\mathcal{M}^{\prime}, \mathcal{M}\right\} \rightarrow \mathcal{N}$ with $\mathcal{M}^{\prime}$ being strongly $\operatorname{Jets}(G)$ equivariant, we on the one hand, obtain a chiral pairing of complexes

$$
\left\{\mathcal{M}^{\prime}, \mathcal{F}_{2}^{\prime} \star \mathcal{N}\right\} \rightarrow \mathcal{F}_{2}^{\prime} \star \mathcal{N},
$$

from which we further obtain a chiral pairing of bi-complexes

$$
\left\{\mathcal{N}^{\prime}, \mathcal{F}_{1}^{\prime} \star\left(\mathcal{F}_{2}^{\prime} \star \mathcal{M}\right)\right\} \rightarrow \mathcal{F}_{1}^{\prime} \star\left(\mathcal{F}_{2}^{\prime} \star \mathcal{N}\right) \text {. }
$$

On the other hand, from the original pairing we obtain another pairing of bicomplexes

$$
\left\{\mathcal{M}^{\prime},\left(\mathcal{F}_{1}^{\prime} \star \mathcal{F}_{2}^{\prime}\right) \star \mathcal{M}\right\} \rightarrow\left(\mathcal{F}_{1}^{\prime} \star \mathcal{F}_{2}^{\prime}\right) \star \mathcal{N} .
$$

However, by [FG2], Sect. 22.9.1, the complexes associated to

$$
\left(\mathcal{F}_{1}^{\prime} \star \mathcal{F}_{2}^{\prime}\right) \star \mathcal{M}^{\prime \prime} \text { and } \mathcal{F}_{1}^{\prime} \star\left(\mathcal{F}_{2}^{\prime} \star \mathcal{M}^{\prime \prime}\right)
$$

for $\mathcal{M}^{\prime \prime}=\mathcal{M}$ or $\mathcal{M}^{\prime \prime}=\mathcal{N}$ are isomorphic. The next assertion follows from the construction:

Lemma 4.12. Under the above circumstances, the diagram of complexes

$$
\begin{array}{r}
j_{*} j^{*}\left(\mathcal{M}^{\prime} \otimes\left(\mathcal{F}_{1}^{\prime} \star\left(\mathcal{F}_{2}^{\prime} \star \mathcal{M}\right)\right)\right) \\
\sim \downarrow \\
\downarrow \\
\left.j_{*} j^{*}\left(\mathcal{M}^{\prime} \otimes\left(\left(\mathcal{F}_{1}^{\prime} \star \mathcal{F}_{2}^{\prime}\right) \star \mathcal{M}\right)\right)\right)
\end{array}
$$

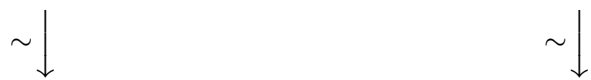

commutes.

4.13. Proof of Theorem 2.6. We shall now prove the following generalization of Theorem 2.6. Let $\mathcal{A}$ be a chiral algebra as in Sect. 4.1. Let us assume that the level $\kappa$ is integral, i.e., the spherical D-modules $\mathcal{F}_{V, X}$ on $\operatorname{Gr}_{G, X}$ for $V \in \operatorname{Rep}(\check{G})$ make sense.

Assume that for any $V$ as above the convolution $\mathcal{F}_{V, X} \star \mathcal{A}$ is acyclic away from degree 0 . 
Theorem 4.14. Let $\mathcal{M}$ be a strongly $\operatorname{Jets}(G)_{X}$-equivariant chiral module.

(1) The convolution $\mathcal{F}_{V, X} \star \mathcal{M}$ is acyclic away from cohomological degree 0 .

(2) Chiral pairings $\left\{\mathcal{F}_{V, X} \star \mathcal{A}, \mathcal{N}\right\} \rightarrow \mathcal{N}$, where $\mathcal{N}$ is any other chiral $\mathcal{A}$-module, are in bijection with maps of chiral $\mathcal{A}$-modules $\mathcal{F}_{V, X} \star \mathcal{M} \rightarrow \mathcal{N}$.

In this subsection we will prove the first point of the theorem.

The functor $\mathcal{M} \mapsto \mathcal{F}_{V, X} \star \mathcal{M}$ on the derived category of strongly $\operatorname{Jets}(G)_{X^{-}}$ equivariant chiral modules over $\mathcal{A}$ is both left and right adjoint to $\mathcal{N} \mapsto \mathcal{F}_{V^{*}, X} \star \mathcal{N}$. Hence, it is enough to show that it is right-exact. Suppose not, and let $k$ be the maximal integer, for which $h^{k}\left(\mathcal{F}_{V, X} \star \mathcal{M} \mathcal{H}\right)$ is non-zero for some $\mathcal{M} \in \mathcal{A}$-mod $\operatorname{Jets}(G)_{X}$. Then $k$ is also the maximal integer, for which $h^{-k}\left(\mathcal{F}_{V^{*}, X} \star \mathcal{N}\right) \neq 0$ for $\mathcal{N} \in$ $\mathcal{A}$-mod ${ }^{\operatorname{Jets}(G)_{X}}$.

Let us choose an object $\mathcal{M}$ as above that saturates this bound, and let us denote by $\mathcal{N}$ the $k$-th cohomology of $\mathcal{F}_{V, X} \star \mathcal{M}$. By adjunction we have a non-zero $\operatorname{map} \mathcal{M} \rightarrow h^{-k}\left(\mathcal{F}_{V^{*}, X} \star \mathcal{N}\right)$.

We can represent $\tau^{\geq 0}\left(\mathcal{F}_{V, X} \star \mathcal{M}\right)$ by a complex $\mathcal{M}_{1}^{\bullet}$, supported in degrees $\geq 0$, such that the map of (43) gives rise to a chiral pairing $\left\{\mathcal{F}_{V, X} \star \mathcal{A}, \mathcal{M}\right\} \rightarrow \mathcal{M}_{1}^{\bullet}$. Moreover, we can represent $\mathcal{N}$ by a complex $\mathcal{N} \bullet$, also supported in degrees $\geq 0$, such that the map $\mathcal{F}_{V, X} \star \mathcal{M} \rightarrow \mathcal{N}[-k]$ is represented by a map of complexes $\mathcal{M}_{i}^{\bullet} \rightarrow \mathcal{N}^{\bullet}[-k]$.

Consider the diagram of complexes:

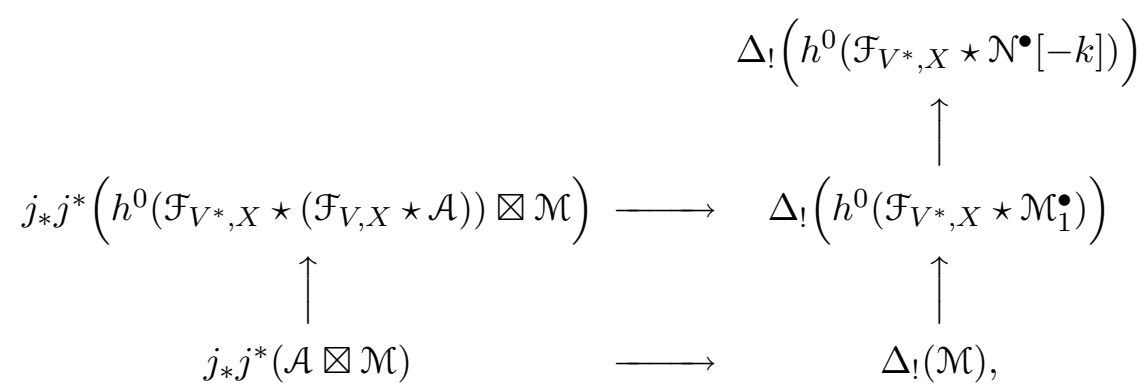

where the left vertical arrow comes from the map $\delta_{1, \mathrm{Gr}_{G}} \rightarrow \mathcal{F}_{V^{*}, X} \star \mathcal{F}_{V, X}$, the lower right vertical arrow comes from

$$
\mathcal{M} \rightarrow \mathcal{F}_{V^{*}, X} \star \mathcal{F}_{V, X} \star \mathcal{M} \rightarrow \mathcal{F}_{V^{*}, X} \star \mathcal{M}_{1},
$$

and the upper horizontal arrow comes by functoriality from PropositionConstruction 4.8. This diagram is commutative by Lemma 4.12 . 
The composed arrow from the lower left corner to the upper right corner of the above diagram vanishes, since the composition

$$
j_{*} j^{*}\left(\left(\mathcal{F}_{V, X} \star \mathcal{A}\right) \otimes \mathcal{M}\right) \rightarrow \Delta_{!}\left(\mathcal{M}_{1}^{\bullet}\right) \rightarrow \mathcal{N}^{\bullet}[-k]
$$

is evidently equal to 0 .

This is a contradiction, since the lower horizontal arrow is surjective, and the composition

$$
\Delta_{!}(\mathcal{M}) \rightarrow \Delta_{!}\left(h^{0}\left(\mathcal{F}_{V^{*}, X} \star \mathcal{M}_{1}\right)\right) \rightarrow \Delta_{!}\left(h^{0}\left(\mathcal{F}_{V^{*}, X} \star \mathcal{N}^{\bullet}[-k]\right)\right)
$$

equals the map $\mathcal{M} \rightarrow h^{-k}\left(\mathcal{F}_{V^{*}, X} \star \mathcal{N}\right)$ above.

4.15. Proof of Theorem 4.14(2). The canonical map of (44) assigns to every map of chiral modules $\mathcal{F}_{V, X} \star \mathcal{M} \mathfrak{\mathcal { N }} \rightarrow \mathcal{N}$ a chiral pairing $\phi:\left\{\mathcal{F}_{V, X} \star \mathcal{A}, \mathcal{M}\right\} \rightarrow \mathcal{F}_{V, X} \star \mathcal{M}$.

Let us construct a map in the opposite direction. By Proposition 4.6, it is sufficient to consider the case when $\mathcal{N}$ is also $\operatorname{Jets}(G)_{X}$-equivariant. For $\phi$ as above, consider the chiral pairing

$$
j_{*} j^{*}\left(\left(\mathcal{F}_{V^{*}, X} \star \mathcal{F}_{V, X} \star \mathcal{A}\right) \otimes \mathcal{M}\right) \rightarrow \Delta_{!}\left(\mathcal{F}_{V^{*}, X} \star \mathcal{N}\right),
$$

obtained from Proposition-Construction 4.8. Using the canonical map $\mathcal{A} \rightarrow$ $\mathcal{F}_{V^{*}, X} \star \mathcal{F}_{V, X} \star \mathcal{A}$ we thus obtain a chiral pairing $\{\mathcal{A}, \mathcal{M}\} \rightarrow \mathcal{F}_{V^{*}, X} \star \mathcal{N}$. By Lemma 2.2, the latter gives rise to a map of chiral modules $\mathcal{M} \rightarrow \mathcal{F}_{V^{*}, X} \star \mathcal{N}$. By adjunction, we obtain a map $\psi: \mathcal{F}_{V, X} \star \mathcal{M} \rightarrow \mathcal{N}$, as required.

The fact that, if the initial chiral pairing $\phi$ came from a map $\mathcal{F}_{V, X} \star \mathcal{M} \rightarrow \mathcal{N}$, then the resulting map $\psi$ equals the initial one, follows from (46).

Let us start with a map $\phi$, and show that the pairing $\phi^{\prime}:\left\{\mathcal{F}_{V, X} \star \mathcal{A}, \mathcal{M}\right\} \rightarrow \mathcal{M}$ obtained from the corresponding $\psi$, equals the initial $\phi$.

Consider the three maps

$$
j_{*} j^{*}\left(\mathcal{A} \otimes\left(\mathcal{F}_{V, X} \star \mathcal{A}\right) \otimes \mathcal{M}\right) \rightarrow \Delta_{!}(\mathcal{N}),
$$

obtained from the map $\phi$. By the definition of chiral pairings, their sum equals 0. Using Proposition-Construction 4.8, we obtain three maps

$$
j_{*} j^{*}\left(\mathcal{A} \otimes\left(\mathcal{F}_{V^{*}, X} \star \mathcal{F}_{V, X} \star \mathcal{A}\right) \otimes \mathcal{M}\right) \rightarrow \Delta_{!}\left(\mathcal{F}_{V^{*}, X} \star \mathcal{N}\right),
$$

whose sum is still equal to 0, by Lemma 4.11.

Furthermore, using Proposition-Construction 4.8 again, we obtain three maps

$$
j_{*} j^{*}\left(\left(\mathcal{F}_{V, X} \star \mathcal{A}\right) \otimes\left(\mathcal{F}_{V^{*}, X} \star \mathcal{F}_{V, X} \star \mathcal{A}\right) \otimes \mathcal{M}\right) \rightarrow \Delta_{!}\left(\mathcal{F}_{V, X} \star \mathcal{F}_{V^{*}, X} \star \mathcal{N}\right) .
$$


Composing these maps with $\mathcal{A} \rightarrow \mathcal{F}_{V^{*}, X} \star \mathcal{F}_{V, X} \star \mathcal{A}$ and $\mathcal{F}_{V, X} \star \mathcal{F}_{V^{*}, X} \star \mathcal{N} \rightarrow \mathcal{N}$ we obtain three maps

$$
j_{*} j^{*}\left(\left(\mathcal{F}_{V, X} \star \mathcal{A}\right) \otimes \mathcal{A} \otimes \mathcal{M}\right) \rightarrow \Delta_{!}(\mathcal{N}),
$$

that sum up to zero.

Let us calculate the resulting maps explicitly. It is easy to see that the first of these maps, namely, the one obtained by first fusing the $x_{1}$ and $x_{2}$ coordinates equals

$$
j_{*} j^{*}\left(\left(\mathcal{F}_{V, X} \star \mathcal{A}\right) \otimes \mathcal{A} \otimes \mathcal{M}\right) \rightarrow \Delta_{x_{1}=x_{2} !}\left(j_{*} j^{*}\left(\left(\mathcal{F}_{V, X} \star \mathcal{A}\right) \otimes \mathcal{M}\right)\right) \stackrel{\phi}{\rightarrow} \Delta_{!}(\mathcal{N}),
$$

where the first arrow is given by the chiral action of $\mathcal{A}$ on $\mathcal{F}_{V, X} \star \mathcal{A}$.

The second map, namely, the one obtained by first fusing the $x_{2}$ and $x_{3}$ coordinates equals

$$
j_{*} j^{*}\left(\left(\mathcal{F}_{V, X} \star \mathcal{A}\right) \otimes \mathcal{A} \nabla \mathcal{M}\right) \rightarrow \Delta_{x_{2}=x_{3} !}\left(j_{*} j^{*}\left(\left(\mathcal{F}_{V, X} \star \mathcal{A}\right) \otimes \mathcal{M}\right)\right) \stackrel{\phi^{\prime}}{\rightarrow} \Delta_{!}(\mathcal{N}),
$$

where the first arrow is the chiral action of $\mathcal{A}$ on $\mathcal{M}$.

The third arrow, by construction, factors through a D-module supported on the diagonal $x_{1}=x_{3}$.

Let us now compare the three maps of (47) with the three maps between the same objects that correspond to the initial chiral pairing $\phi$, and subtract one from another.

We have two equal maps

$$
j_{*} j^{*}\left(\left(\mathcal{F}_{V, X} \star \mathcal{A}\right) \otimes \mathcal{A} \otimes \mathcal{M}\right) \rightarrow \Delta_{!}(\mathcal{N}),
$$

such that one factors as

$$
j_{*} j^{*}\left(\left(\mathcal{F}_{V, X} \star \mathcal{A}\right) \otimes \mathcal{A} \otimes \mathcal{M}\right) \rightarrow \Delta_{x_{2}=x_{3} !}\left(j_{*} j^{*}\left(\left(\mathcal{F}_{V, X} \star \mathcal{A}\right) \otimes \mathcal{M}\right)\right) \stackrel{\phi^{\prime}-\phi}{\longrightarrow} \Delta_{!}(\mathcal{N}),
$$

and the other through a D-module, supported on the diagonal $x_{1}=x_{3}$. this implies that both maps are in fact 0 , and in particular, $\phi=\phi^{\prime}$.

\section{Part II: The Iwahori case}

In this part of the paper we consider convolutions of the central sheaves on the affine flag variety with objects of the category of $I$-equivariant $\widehat{\mathfrak{g}}_{\text {crit-modules. }}$ - 


\section{Convolution with Central sheaves}

5.1. Opers with nilpotent singularities. From now on we shall fix a point $x \in X$. Let $\lambda$ be an integral weight such that $\lambda+\rho$ is dominant. Recall that to such $\lambda$ in [FG2], Sect. 7.6, we have attached a subscheme

$$
\operatorname{Spec}\left(\mathfrak{Z}_{\mathfrak{g}}^{\lambda, \text { nilp }}\right) \subset \operatorname{Spec}\left(\mathfrak{Z}_{\mathfrak{g}}\right)
$$

In terms of the isomorphism $(7)$ between $\operatorname{Spec}\left(\mathfrak{Z}_{\mathfrak{g}}\right)$ and the ind-scheme of $\check{\mathfrak{g}}$ opers on $\mathcal{D}_{x}^{\times}$, the subscheme $\operatorname{Spec}\left(\mathfrak{Z}_{\mathfrak{g}}^{\lambda \text {,nilp }}\right)$ corresponds to opers with a regular singularity and residue $\varpi(-\lambda-\rho)$, see [FG2], Sect. 2.9.

According to [FG2], Sect. 7.6, if a weight $\mu$ is of the form $w(\lambda+\rho)-\rho$ for some $w \in W$, then the support of the $\widehat{\mathfrak{g}}_{\text {crit }}$-module $\mathbb{M}^{\mu}:=\operatorname{Ind}_{\mathfrak{g}[t[t]]}^{\widehat{\mathfrak{g}}_{\text {crit }}}\left(M^{\mu}\right)$, where $M^{\mu}$ denotes the Verma module of highest weight $\mu$ over $\mathfrak{g}$, is contained (and in fact equal to) $\operatorname{Spec}\left(\mathfrak{Z}_{\mathfrak{g}}^{\lambda \text {,nilp }}\right)$.

Let $\mathfrak{z}_{\mathfrak{g}}^{\lambda \text {,nilp }}$ be the modification of the D-algebra $\mathfrak{z}_{\mathfrak{g}}$ at $x$, corresponding to $\mathfrak{Z}_{\mathfrak{g}}^{\lambda \text {,nilp }}$. From [FG2], Sect. 2.9, we obtain that for every $V \in \operatorname{Rep}(\breve{G})$, the module $\mathcal{V}_{X-x}$ extends naturally to a free $\mathfrak{Z}_{\mathfrak{g}}^{\lambda \text {,nilp }}$-module, such that the connection has a pole of order $\leq 1$ at $x$ and a nilpotent monodromy.

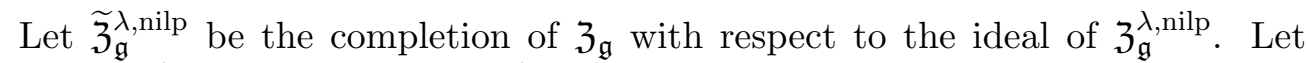
$\operatorname{Spec}\left(\mathfrak{Z}_{\mathfrak{g}}^{\text {int,nilp }}\right)\left(\right.$ resp., $\left.\operatorname{Spec}\left(\widetilde{\mathfrak{Z}}_{\mathfrak{g}}^{\text {int,nilp }}\right)\right)$ be the sub-ind scheme of $\operatorname{Spec}\left(\mathfrak{Z}_{\mathfrak{g}}\right)$ defined

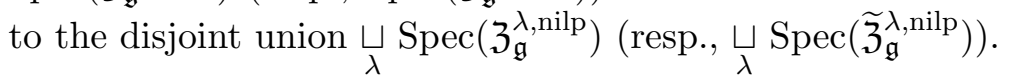

Applying Lemma 1.4, we obtain that for each $V \in \operatorname{Rep}(\check{G})$, the module $\mathcal{V}_{X-x}$ gives rise to a vector bundle $\mathcal{V}_{\widetilde{\mathfrak{Z}}_{\mathfrak{g}}^{\text {int,nilp }}}$ over $\operatorname{Spec}\left(\widetilde{\mathfrak{Z}}_{\mathfrak{g}}^{\text {int,nilp }}\right)$, equipped with a nilpotent endomorphism, which we will denote by $N_{\mathcal{V}_{\widetilde{\mathfrak{z}}_{\mathfrak{g}} \text { int,nilp }}}$. Moreover, for $U \simeq V \otimes W$ we have an isomorphism

$$
\mathcal{U}_{\widetilde{\mathfrak{Z}}_{\mathfrak{g}}^{\text {int,nilp }}} \simeq \mathcal{V}_{\widetilde{\mathfrak{Z}}_{\mathfrak{g}}^{\text {int,nilp }}} \underset{\widetilde{\mathfrak{Z}}_{\mathfrak{g}}^{\text {int,nilp }}}{\otimes} \mathcal{W}_{\widetilde{\mathfrak{Z}}_{\mathfrak{g}}^{\text {int,nilp }}}
$$

so that $N_{\mathcal{U}_{\widetilde{\mathfrak{Z}}_{\mathfrak{g}} \text { int,nilp }}}=N_{\mathcal{V}_{\widetilde{\mathfrak{Z}}_{\mathfrak{g}}} \text { int,nilp }}+N_{\mathcal{W}_{\widetilde{\mathfrak{z}}_{\mathfrak{g}} \text { int,nilp }}}$.

5.2. Statement of the theorem. Let $I \subset G((t))$ be the Iwahori subgroup, i.e., the preimage of $B \subset G$ under the evaluation map $G[[t]] \rightarrow G$. Following the notation of [FG2], we shall denote by $\widehat{\mathfrak{g}}_{\kappa}$-mod ${ }^{I}$ the category of $I$-integrable representations of $\widehat{\mathfrak{g}}$ at the level $\kappa$.

The above estimate on the support of the modules $\mathbb{M}^{\mu}$ implies: 
Lemma 5.3. The support of every object $\mathcal{M} \in \widehat{\mathfrak{g}}_{\text {crit }}$-mod ${ }^{I}$ over $\operatorname{Spec}\left(\mathfrak{Z}_{\mathfrak{g}}\right)$ is contained in $\operatorname{Spec}\left(\widetilde{\mathfrak{Z}}_{\mathfrak{g}}^{\text {int,nilp }}\right)$.

Thus, for every $\mathcal{M} \in \widehat{\mathfrak{g}}_{\text {crit }}-\bmod { }^{I}$ and $V \in \operatorname{Rep}(\check{G})$ we can functorially attach another object of $\widehat{\mathfrak{g}}_{\text {crit }}-\bmod { }^{I}$ :

$$
\mathcal{V}_{\widetilde{\mathfrak{Z}}_{\mathfrak{g}}^{\text {int,nilp }}} \underset{\widetilde{\mathfrak{Z}}_{\mathfrak{g}}^{\text {int,nilp }}}{\otimes} \mathcal{M}
$$

which carries a nilpotent endomorphism $N_{\mathcal{V}_{\tilde{\mathfrak{J}}_{\mathfrak{g}} \text { int,nilp }}}$.

Let $\mathrm{Fl}_{G}=G((t)) / I$ be the affine flag scheme of $G$. For a level $\kappa$ we shall denote by $\mathrm{D}\left(\mathrm{Fl}_{G}\right)_{\kappa}$-mod the category of $\kappa$-twisted right D-modules on $\mathrm{Fl}_{G}$. By $\mathrm{D}\left(\mathrm{Fl}_{G}\right)_{\kappa}$-mod ${ }^{I}$ we shall denote the corresponding category of $I$-equivariant objects in $\mathrm{D}\left(\mathrm{Fl}_{G}\right)_{\kappa}$-mod. When $\kappa$ is integral (e.g., critical) we will sometimes identify $\mathrm{D}\left(\mathrm{Fl}_{G}\right)_{\kappa}$-mod with the usual category of $\mathrm{D}$-modules by means of the tensor product with the corresponding line bundle.

Given an object $\mathcal{F} \in \mathrm{D}\left(\mathrm{Fl}_{G}\right)_{\kappa}$-mod and $\mathcal{M} \in \mathfrak{g}_{\kappa}$-mod ${ }^{I}$ we can form their convolution, denoted $\mathcal{F} \star \mathcal{M}$, which is an object of $D\left(\mathfrak{g}_{\kappa}\right.$-mod $)$. When no confusion is likely to occur, we will omit the subscript $I$ from $\underset{I}{\star}$.

Let us recall from [Ga] that to every object $V \in \operatorname{Rep}(\breve{G})$ there corresponds an object $z_{V} \in \mathrm{D}\left(\mathrm{Fl}_{G}\right)$-mod ${ }^{I}$ called a central sheaf. Each central sheaf is endowed with a functorial endomorphism $N_{V}$. The construction of D-modules $z_{V}$ will be reviewed in some detail in the sequel. Slightly abusing the notation, we shall denote by the same symbol $z_{V}$ the corresponding $I$-equivariant object of $\mathrm{D}\left(\mathrm{Fl}_{G}\right)_{\text {crit }}-\bmod ^{I}$.

Our main result is the following:

Theorem 5.4. For every $\mathcal{M} \in \widehat{\mathfrak{g}}_{\text {crit }}$-mod ${ }^{I}$ and $V \in \operatorname{Rep}(\check{G})$ the convolution $z_{V} \star \mathcal{M}$ is acyclic away from cohomological degree 0 , and we have a canonical isomorphism

$$
\mathfrak{s}_{V}: Z_{V}^{\star} \underset{I}{ } \mathcal{M} \simeq \mathcal{V}_{\widetilde{\mathfrak{Z}}_{\mathfrak{g}, x}^{\text {int,nilp }}} \underset{\widetilde{\mathfrak{Z}}_{\mathfrak{g}, x}^{\text {int,nilp }}}{\otimes} \mathcal{M}
$$

such that the endomorphism induced by $N_{V}$ on the LHS goes over to the endomorphism, induced by $N_{\mathcal{V}_{\widetilde{\mathfrak{z}}}^{\text {int,nilp }}}$ on the RHS. This system of isomorphisms is compatible with tensor products of $\breve{G-r e p r e s e n t a t i o n s ~ i n ~ t h e ~ s a m e ~ s e n s e ~ a s ~ i n ~}$ Theorem 1.10 
5.5. Spherical case, revisited. Let us note that Theorem 5.4, whose prove is parallel to, but independent of, the proof of Theorem 1.10, implies the latter theorem. Indeed, let $\mathfrak{p}$ denote the natural projection $\mathrm{Fl}_{G} \rightarrow \mathrm{Gr}_{G}$; then by [Ga], there is a canonical isomorphism

$$
\mathfrak{p}_{!}\left(\mathcal{Z}_{V}\right) \simeq \mathcal{F}_{V} .
$$

For an object $\mathcal{M} \in \widehat{\mathfrak{g}}_{\text {crit }}$-mod ${ }^{G[t]]}$ we have:

$$
z_{V} \underset{I}{\star} \mathcal{M} \simeq \mathcal{F}_{V} \underset{G[[t]]}{\star} \mathcal{N}
$$

and the assertion follows from the fact that the restriction of $\mathcal{V}_{\widetilde{\mathfrak{Z}}_{\mathfrak{g}, x}^{\text {int,nilp }}}$ to $\operatorname{Spec}\left(\widetilde{\mathfrak{Z}}_{\mathfrak{g}}^{\text {int,reg }}\right) \subset \operatorname{Spec}\left(\widetilde{\mathfrak{Z}}_{\mathfrak{g}, x}^{\text {int,nilp }}\right)$ identifies canonically with $\mathcal{V}_{\widetilde{\mathfrak{z}}_{\mathfrak{g}, x} \text { int,reg }}$

In particular, since the nilpotent endomorphism that $N_{V}$ induces on $\mathcal{F}_{V}$ is zero, we obtain the assertion of Corollary 1.11. This, in turn, gives an alternative proof of Lemma 1.8, as was promised earlier. Let us now prove Lemma 1.7:

Proof. One the one hand, by [FG2], Corollary 7.6.2, the support of $\mathbb{V}^{\mu}$ over $\operatorname{Spec}\left(\mathfrak{Z}_{\mathfrak{g}}\right)$ is contained in the subscheme $\operatorname{Spec}\left(\mathfrak{Z}_{\mathfrak{g}}^{\mu, \text { nilp }}\right)$. On the other hand, by Corollary 1.11, which was proved independently, the support of $\mathbb{V}^{\mu}$ is contained in the ind-subscheme $\operatorname{Spec}\left(\mathfrak{Z}_{\mathfrak{g}}^{\text {m.f. }}\right)$. The assertion of the lemma follows now from the fact that

$$
\operatorname{Spec}\left(\mathfrak{Z}_{\mathfrak{g}}^{\mu, \text { nilp }}\right) \cap \operatorname{Spec}\left(\mathfrak{Z}_{\mathfrak{g}}^{\text {m.f. }}\right)=\operatorname{Spec}\left(\mathfrak{Z}_{\mathfrak{g}}^{\mu, \text { reg }}\right),
$$

(see [FG2], Sect. 2.9).

5.6. The case of differential operators. Parallel to the spherical situation, let us consider a particular case of the above theorem, corresponding to differential operators. Consider the object of $\delta_{I, G((t))} \in \mathfrak{D}_{G, \text { crit- }}-\bmod _{x}$, corresponding to distributions on $I$.

In other words, if $\pi_{\mathrm{Fl}}$ denotes the projection $G((t)) \rightarrow \mathrm{Fl}_{G}$, then

$$
\delta_{I, G((t))} \simeq \Gamma\left(G((t)), \pi_{\mathrm{Fl}}^{*}\left(\delta_{1, \mathrm{Fl}_{G}}\right)\right),
$$

where $\delta_{1, \mathrm{Fl}_{G}}$ is the $\delta$-function at $1 \in \mathrm{Fl}_{G}$. As a $\widehat{\mathfrak{g}}_{\text {crit-module, }} \delta_{I, G((t))}$ can be described as $\operatorname{Ind}_{\mathfrak{g}[t[t]}^{\widehat{\mathfrak{a}}_{\mathrm{crit}}}\left(\mathcal{O}_{I}\right)$. We have:

Theorem 5.7. We have a canonical isomorphism of $\widehat{\mathfrak{g}}_{\text {crit }}$-bimodules

$$
\Gamma\left(G((t)), \pi_{\mathrm{Fl}_{G}}^{*}\left(z_{V}\right)\right) \simeq \nu_{\widetilde{\mathfrak{Z}}_{\mathfrak{g}, x}^{\text {int,nilp }}} \underset{\widetilde{\mathfrak{Z}}_{\mathfrak{g}, x}^{\text {int,nilp }}}{\otimes} \delta_{I, G((t))},
$$

which intertwines the endomorphism $N_{V}$ on the LHS with $N_{{\mathcal{\mathcal { Z } _ { \mathfrak { g } }}}_{\text {int,nilp }}}$ on the RHS. This system of isomorphisms is compatible with tensor products of $\breve{G}$-representations. 
The same argument as in the spherical case shows that Theorem 5.7 actually implies Theorem 5.4.

\section{Fusion in the Iwahori CASE}

Our strategy of proof of Theorem 5.4 will be parallel to that of Theorem 1.10. Namely, we will construct a functor on the category of $\widehat{\mathfrak{g}}_{\text {crit-modules, and show }}$ that it is represented by both sides of the isomorphism stated in the theorem.

An additional ingredient in the present situation is that we will have to twist the chiral $\mathcal{A}_{\mathfrak{g}, \kappa}$-modules $\Gamma\left(\operatorname{Gr}_{G, X-x}, \mathcal{F}_{V, X-x}\right)$ by certain local systems on the punctured curve.

6.1. Nilpotent local systems. Let us fix a family of local systems $\mathcal{E}_{n}, n \in \mathbb{N}$, defined on a punctured Zariski neighborhood of $x \in X$, each $\mathcal{E}_{n}$ being an $n$-fold extension of the trivial local system (i.e., $\mathcal{E}_{1}$ is the trivial local system), endowed with a nilpotent endomorphism $N_{\mathcal{E}}$ of order $n-1$, and such that we are given a compatible system of surjections

$$
\mathcal{E}_{n} \rightarrow \mathcal{E}_{m}
$$

defined for every $m \leq n$ and compatible with the action of $N_{\mathcal{E}}$. Then the action of $N_{\mathcal{E}}$ gives rise to a system of exact sequences:

$$
0 \rightarrow \mathcal{E}_{m} \rightarrow \mathcal{E}_{n} \stackrel{N_{\varepsilon}^{m}}{\rightarrow} \mathcal{E}_{n} \rightarrow \mathcal{E}_{n-m} \rightarrow 0
$$

For example, if we choose a local coordinate $t$ near $x$, such a system can be obtained as follows. Let $E_{n}$ be the standard $n$-dimensional Jordan block, i.e., the vector space $\mathbb{C}[s] / s^{n-1}$ with the nilpotent operator $N_{E}$ equal to the multiplication by $s$. Set $\mathcal{E}_{n}:=E_{n} \otimes \mathcal{O}_{X-x}$, with $t \nabla_{t}$ acting as $N_{E}+t \partial_{t}$.

Let us denote by $\varepsilon_{n, x}$ the fiber at $x$ of the extension of $\varepsilon_{n}$, given by Lemma 1.4. This is an $n$-dimensional vector space, endowed with a nilpotent operator of order $n-1$, which coincides with the one induced by $N_{\mathcal{E}}$. We shall fix a system of identifications $\mathcal{E}_{n, x} \simeq E_{n}$, such that $N_{\mathcal{E}}$ goes over to $N_{E}$, and which is compatible with the morphisms (48).

6.2. A canonical chiral pairing. Recall now that if $\mathcal{A}$ is any chiral algebra and $\mathcal{M}$ is a chiral module over it, and $\mathcal{E}$ is any D-module on $X$, then the D-module $\mathcal{M} \otimes \mathcal{E}$ is naturally a chiral module over $\mathcal{A}$.

Proposition-Construction 6.3. Let $\kappa$ be any integral level. Then for any $\mathcal{M} \in$ $\widehat{\mathfrak{g}}_{\kappa}-\bmod ^{I}$ and $n \in \mathbb{N}$ there exists a canonical chiral pairing of $\mathfrak{D}_{G \text {,crit-modules: }}$

$$
j_{x *} j_{x}^{*}\left(\Gamma\left(G((t)), \pi^{*}\left(\mathcal{F}_{V}\right)\right) \otimes \mathcal{E}_{n}\right) \otimes \delta_{I, G((t))} \rightarrow i_{x !}\left(\Gamma\left(G((t)), \pi_{\mathrm{Fl}}^{*}\left(z_{V}\right)\right)\right)_{N_{V}^{n}},
$$


such that for $n \geq m$ the diagram

$$
\begin{gathered}
j_{x *} j_{x}^{*}\left(\Gamma\left(G((t)), \pi^{*}\left(\mathcal{F}_{V}\right)\right) \otimes \mathcal{E}_{n}\right) \otimes \delta_{I, G((t))} \longrightarrow i_{x !}\left(\Gamma\left(G((t)), \pi_{\mathrm{Fl}}^{*}\left(z_{V}\right)\right)\right)_{N_{V}^{n}} \\
\downarrow \\
j_{x *} j_{x}^{*}\left(\Gamma\left(G((t)), \pi^{*}\left(\mathcal{F}_{V}\right)\right) \otimes \mathcal{E}_{m}\right) \otimes \delta_{I, G((t))} \longrightarrow i_{x !}\left(\Gamma\left(G((t)), \pi_{\mathrm{Fl}}^{*}\left(z_{V}\right)\right)\right)_{N_{V}^{m}}
\end{gathered}
$$

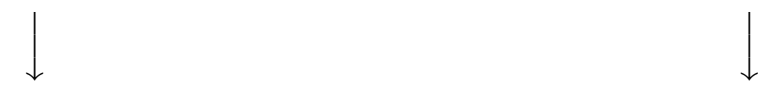

is commutative.

Before giving the proof we need to review the construction of the D-modules $z_{V}$. Consider the ind-scheme $\mathrm{Fl}_{G, X}$ over $X$, whose fiber over $x_{1} \in X$ is the set of triples $\left(\mathcal{P}_{G}, \beta, \alpha\right)$, where $\beta$ is a trivialization of $\mathcal{P}_{G}$ on $X-\left\{x, x_{1}\right\}$ and $\alpha$ is a reduction of the fiber $\mathcal{P}_{G, x}$ at $x$ to $B$. Note that $\mathrm{Fl}_{G, X}$ is the same as $\operatorname{Gr}_{G, X ; K}$ for $K=I$.

The preimage of $X-x$ in $\mathrm{Fl}_{G, X}$, denoted $\mathrm{Fl}_{G, X-x}$, is isomorphic to $\mathrm{Gr}_{G, X-x} \times \mathrm{Fl}_{G}$, whereas the preimage of $x \in X$ is isomorphic to $\mathrm{Fl}_{G}$. More generally, we will consider the ind-scheme $\mathrm{Fl}_{G, X^{n}}$ over $X^{n}$, whose fiber over $\left(x_{1}, \ldots, x_{n}\right) \in X^{n}$ is the set of triples $\left(\mathcal{P}_{G}, \beta, \alpha\right)$, where $\beta$ now is a trivialization defined away from $x_{1} \cup \ldots \cup x_{n} \cup x$.

Recall the ind-scheme Jets ${ }^{\operatorname{mer}}(G)_{X^{n}}$ from Sect. 2.9, and let Jets ${ }^{\operatorname{mer}}(G)_{X^{n-1} \times x}$ be the closed ind-subscheme defined by the condition that the last point in the $n$-tuple $x_{1}, \ldots, x_{n}$ is fixed to be $x$.

Note that we have a natural projection $\pi_{\mathrm{Fl}}: \operatorname{Jets}^{\mathrm{mer}}(G)_{X^{n} \times x} \rightarrow \mathrm{Fl}_{G, X^{n}}$, which corresponds to remembering the reduction to $B$ at $x$ out of the trivialization of $\mathcal{P}_{G}$ on the formal neighborhood of $x_{1} \cup \ldots \cup x_{n} \cup x$.

Given an object $V \in \operatorname{Rep}(\check{G})$ and $n \in \mathbb{N}$, we consider $\left(\mathcal{F}_{V, X-x} \otimes \mathcal{E}_{n}\right) \otimes \delta_{1, \mathrm{Fl}_{G}}$ as a D-module on $\mathrm{Fl}_{G, X-x}$. For $n \in \mathbb{N}$ consider the fiber over $x$ of its intermediate extension onto the entire $\mathrm{Fl}_{G, X}$, i.e.,

$$
z_{V, n}:=i_{x}^{!} j_{x ! *}\left(\left(\mathcal{F}_{V, X-x} \otimes \mathcal{E}_{n}\right) \otimes \delta_{1, \mathrm{Fl}_{G}}\right) .
$$

This is a D-module on $\mathrm{Fl}_{G}$, endowed with an action of $N_{\mathcal{E}}$, by the transport of structure. The following summarizes the main construction of $[\mathrm{Ga}]$ :

- For $n$ large enough and $n^{\prime} \geq n$ the maps $z_{V, n^{\prime}} \rightarrow z_{V, n}$ are isomorphisms.

- The D-module $z_{V}$ is isomorphic to $z_{V, n}$ for all $n$ large enough. Under this identification, the endomorphism $N_{V}$ equals the one induced by $N_{\mathcal{E}}$.

- For any other $n^{\prime \prime}, z_{V, n^{\prime \prime}} \simeq\left(z_{V}\right)_{N_{V}^{n^{\prime \prime}}}$. 
In fact, the above is a paraphrase of the fact that $z_{V}$ is obtained by applying the functor of unipotent nearby cycles $\Psi^{u n}$ to the D-module $\left(\mathcal{F}_{V, X-x} \otimes \delta_{1, \mathrm{Fl}_{G}}\right)$ on $\mathrm{Fl}_{G, X-x}$.

Note that if $\mathcal{E}^{\prime}$ is any local system on $X-x$ with a nilpotent monodromy around $x$, then the formalism of nearby cycles, developed in [Be] implies that

$$
i_{x}^{!} j_{x ! *}\left(\left(\mathcal{F}_{V, X-x} \otimes \mathcal{E}^{\prime}\right) \otimes \delta_{1, \mathrm{Fl}_{G}}\right)[1] \simeq\left(\mathcal{Z}_{V} \otimes \mathcal{E}_{x}^{\prime}\right)_{N_{V}+N_{\mathcal{E}^{\prime}}},
$$

where $\mathcal{E}_{x}^{\prime}$ is the fiber of the extension of $\mathcal{E}^{\prime}$ across $x$, given by Lemma 1.4, and $N_{\mathcal{E}^{\prime}}$ is its canonical nilpotent endomorphism.

6.4. Proof of Proposition-Construction 6.3. Consider the canonical map of twisted D-modules on $\mathrm{Fl}_{G, X}$

$$
j_{x *}\left(\left(\mathcal{F}_{V, X-x} \otimes \mathcal{E}_{n}\right) \otimes \delta_{1, \mathrm{Fl}_{G}}\right) \rightarrow i_{x !}\left(z_{V, n}\right) .
$$

By taking the pull-back of (50) under $\pi_{\mathrm{Fl}}: \operatorname{Jets}^{\mathrm{mer}}(G)_{X \times x} \rightarrow \mathrm{Fl}_{G, X}$, we obtain a map of D-modules on $X$ :

$$
j_{x *} j_{x}^{*}\left(\Gamma\left(\operatorname{Jets}^{\mathrm{mer}}(G)_{X}, \pi^{*}\left(\mathcal{F}_{V, X}\right)\right) \otimes \mathcal{E}_{n}\right) \otimes \delta_{I, G((t))} \rightarrow i_{x !}\left(\Gamma\left(G((t)), \pi_{\mathrm{Fl}}^{*}\left(\mathcal{Z}_{V}\right)\right)\right)_{N_{V}^{n}},
$$

as was stated in Proposition-Construction 6.3. Thus, in order to finish the proof, we need to show that the above map respects the action of $\mathfrak{D}_{G, \kappa}$, i.e., that it is indeed a chiral pairing.

Consider the ind-scheme $\mathrm{Fl}_{G, X^{2}}$ over $X^{2}$; let $\mathrm{Fl}_{G,(X-x)^{2}-\Delta_{X-x}}$ denote its open subscheme equal to the preimage of the corresponding open subscheme in $X^{2}$; let $j$ denote the corresponding open embedding. Let $\Delta$ be the embedding of $\mathrm{Fl}_{G, x \times x} \simeq \mathrm{Fl}_{G}$.

We have an isomorphism:

$$
\mathrm{Fl}_{G,(X-x)^{2}-\Delta_{X-x}} \simeq\left(\left(\operatorname{Gr}_{G, X-x} \times \operatorname{Gr}_{G, X-x}\right) \underset{(X-x)^{2}}{\times}\left((X-x)^{2}-\Delta_{X-x}\right)\right) \times \mathrm{Fl}_{G} .
$$

As in the case of $\mathrm{Gr}_{G, X^{n}}$, we have a map $\mathbf{1}_{1,1}: X \times \mathrm{Fl}_{G, X} \rightarrow \mathrm{Fl}_{G, X^{2}}$, and consider the twisted D-module on $\mathrm{Fl}_{G, X^{2}}$ equal to

$$
\left(\mathbf{1}_{1,1) !}\left(\omega_{X} \otimes\left(j_{x ! *}\left(\mathcal{F}_{V, X-x} \otimes \mathcal{E}_{n}\right)\right)\right) .\right.
$$

It gives rise to three maps

$$
j_{*} j^{*}\left(\delta_{1, \operatorname{Gr}_{G, X}} \otimes\left(\mathcal{F}_{V, X-x} \otimes \mathcal{E}_{n}\right) \otimes \delta_{1, \mathrm{Fl}_{G}}\right) \rightarrow \Delta_{!}\left(z_{V}\right)_{N_{V}^{n}},
$$

which sum up to zero. 
Pulling back to the two sides of (52) to Jets ${ }^{\operatorname{mer}}(G)_{X^{2} \times x}$ and taking the (quasicoherent) direct image onto $X^{2}$ we obtain three maps

$$
j_{*} j^{*}\left(\mathfrak{D}_{G, \kappa} \otimes\left(\Gamma\left(\operatorname{Jets}^{\mathrm{mer}}(G)_{X}, \pi^{*}\left(\mathcal{F}_{V, X}\right)\right) \otimes \mathcal{E}_{n}\right) \otimes \delta_{I, G((t))}\right) \rightarrow \Delta_{!}\left(\Gamma\left(G((t)), \pi_{\mathrm{Fl}}^{*}\left(z_{V}\right)\right)\right)_{N_{V}^{n}} .
$$

From Proposition 2.12, we obtain that these three maps are equal to those that appear in the definition of chiral pairings.

6.5. Chiral pairings with other representations. As in the spherical situation, from the chiral pairing given by Proposition-Construction 6.3, we obtain a chiral pairing over $\mathcal{A}_{\mathfrak{g}, \kappa}$ :

$$
j_{x *} j_{x}^{*}\left(\left(\Gamma\left(\mathrm{Gr}_{G, X}, \mathcal{F}_{V, X}\right) \otimes \mathcal{E}_{n}\right) \otimes \delta_{I, G((t))} \rightarrow i_{x !}\left(\Gamma\left(G((t)), \pi_{\mathrm{Fl}}^{*}\left(z_{V}\right)\right)\right)_{N_{V}^{n}},\right.
$$

which commutes with the action of $\widehat{\mathfrak{g}}_{\kappa^{\prime}}$ on $\delta_{I, G((t))}$ and $\Gamma\left(G((t)), \pi_{\mathrm{Fl}}^{*}\left(\mathcal{Z}_{V}\right)\right)$, given by $\mathfrak{r}$.

Given an $I$-integrable $\mathfrak{g}_{\kappa}$-module $\mathcal{M}$ we obtain a chiral pairing of complexes of $\mathcal{A}_{\mathfrak{g}, \kappa \text {-modules: }}$

$$
\begin{aligned}
\left\{\Gamma\left(\operatorname{Gr}_{G, X}, \mathcal{F}_{V, X}\right) \otimes \mathcal{E}_{n}, \mathfrak{C}^{\frac{\infty}{2}}\left(\mathfrak{g}((t)) ; \mathfrak{h}, \delta_{I, G((t))} \otimes \mathcal{M}\right)\right\} \rightarrow \\
\rightarrow \mathfrak{C}^{\frac{\infty}{2}}\left(\mathfrak{g}((t)) ; \mathfrak{h}, \Gamma\left(G((t)), \pi_{\mathrm{Fl}}^{*}\left(\mathcal{Z}_{V}\right)\right) \otimes \mathcal{M}\right)_{N_{V}^{n}}
\end{aligned}
$$

In particular, by taking $n>>0$ so that $N_{V}^{n}=0$, we obtain a chiral pairing

$$
\left\{\Gamma\left(\operatorname{Gr}_{G, X-x}, \mathcal{F}_{V, X-x}\right) \otimes \mathcal{E}_{n}, \mathcal{M}\right\} \rightarrow h^{0}\left(\mathcal{Z}_{V} \underset{I}{\star} \mathcal{M}\right),
$$

such that the action of $N_{\mathcal{E}}$ on LHS corresponds to the action of $N_{V}$ on the RHS.

More generally, for an arbitrary local system $\mathcal{E}^{\prime}$ with a nilpotent monodromy around $x$, we obtain a chiral pairing

$$
\left\{\Gamma\left(\operatorname{Gr}_{G, X-x}, \mathcal{F}_{V, X-x}\right) \otimes \mathcal{E}^{\prime}, \mathcal{M}\right\} \rightarrow\left(h^{0}\left(\mathcal{Z}_{V} \underset{I}{\star} \mathcal{M}\right) \otimes \mathcal{E}_{x}^{\prime}\right)_{N_{V}+N_{\mathcal{E}^{\prime}}} .
$$

Parallel to Theorem 2.6, we will prove:

Theorem 6.6. Assume that $\kappa$ is non-positive, Then:

(1) For every $\mathcal{M} \in \widehat{\mathfrak{g}}_{\kappa}-$ mod ${ }^{I}$, the convolution $\boldsymbol{z}_{V} \underset{I}{\star} \mathcal{M}$ is acyclic away from cohomological degree 0 .

(2) The functor on $\widehat{\mathfrak{g}}_{\kappa}$-mod that sends $\mathcal{N}$ to the set of chiral pairings

$$
\left\{\Gamma\left(\mathrm{Gr}_{G, X}, \mathcal{F}_{V, X}\right) \otimes \mathcal{E}^{\prime}, \mathcal{M}\right\} \rightarrow \mathcal{N},
$$

is representable by $\left(\left(\mathcal{Z}_{V} \underset{I}{\star} \mathcal{M}\right) \otimes \mathcal{E}_{x}^{\prime}\right)_{N_{V}+N_{\mathcal{E}^{\prime}}}$. 
We shall now proceed with the proof of Theorem 5.4, which does not rely on Theorem 6.6. We will need to establish the following generalization of Proposition 2.8:

For $V, W \in \operatorname{Rep}(\check{G})$, recall from [Ga] that there exists a natural isomorphism

$$
z_{V} z_{I} z_{W} \simeq z_{V \otimes W}
$$

such that the endomorphism $N_{V}+N_{W}$ on the LHS goes over to the endomorphism $N_{V \otimes W}$.

Let $n, m \in \mathbb{N}$ be such that $z_{V} \rightarrow\left(z_{V}\right)_{N_{V}^{n}}$ and $z_{W} \rightarrow\left(z_{W}\right)_{N_{V}^{n}}$ are isomorphisms. Let $\mathcal{M}$ be an object of $\widehat{\mathfrak{g}}_{\text {crit }}-\bmod { }^{I}$, such that all convolutions $Z_{V^{\prime}} \star \mathcal{M}$, $V^{\prime} \in \operatorname{Rep}(\check{G})$ are acyclic away from cohomological degree 0 .

In this case, as in Proposition 2.8, we have three maps

$j_{*} j^{*}\left(\left(\Gamma\left(\mathrm{Gr}_{G, X}, \mathcal{F}_{V, X}\right) \otimes \mathcal{E}_{n}\right) \otimes\left(\Gamma\left(\operatorname{Gr}_{G, X}, \mathcal{F}_{W, X}\right) \otimes \mathcal{E}_{m}\right) \otimes \mathcal{M}\right) \rightarrow i_{x !}\left(\mathcal{Z}_{V \otimes W} \star \mathcal{M}_{I}\right)$,

defined as follows.

The first map is the composition

$$
\begin{aligned}
& j_{*} j^{*}\left(\left(\Gamma\left(\operatorname{Gr}_{G, X}, \mathcal{F}_{V, X}\right) \otimes \mathcal{E}_{n}\right) \otimes\left(\Gamma\left(\operatorname{Gr}_{G, X}, \mathcal{F}_{W, X}\right) \otimes \mathcal{E}_{m}\right) \otimes \mathcal{M}\right) \rightarrow \\
& \left.\Delta_{x_{2}=x_{3} !}\left(j_{*} j^{*}\left(\Gamma\left(\operatorname{Gr}_{G, X}, \mathcal{F}_{V, X}\right) \otimes \mathcal{E}_{n}\right) \otimes\left(z_{W} \star \mathcal{M}\right)\right)\right) \rightarrow \Delta_{!}\left(z_{V} \underset{I}{\star} z_{W} \underset{I}{\star} \mathcal{M}\right) .
\end{aligned}
$$

The second map is the negative of the one defined by interchanging the roles of $V$ and $W$. To define the third map note that from (49) we obtain a map of D-modules on $\mathrm{Fl}_{G, X}$ :

$$
j_{x *} j_{x}^{*}\left(\left(\Gamma\left(\operatorname{Gr}_{G, X}, \mathcal{F}_{V \otimes W}\right) \otimes \mathcal{E}_{n} \otimes \mathcal{E}_{m}\right) \otimes \delta_{1, \mathrm{Fl}_{G}}\right) \rightarrow \Delta_{!}\left(z_{V}^{\star}{ }_{I} z_{W}\right),
$$

which comes from the projection

$$
\begin{aligned}
& \left(z_{V \otimes W} \otimes E_{n} \otimes E_{m}\right)_{N_{V \otimes W}+\left.N_{\mathcal{E}}\right|_{E_{n}}+\left.N_{\mathcal{E}}\right|_{E_{m}}} \\
& \simeq\left(\left(z_{V} \otimes E_{n}\right) \star\left(z_{W} \otimes E_{m}\right)\right)_{N_{V}+N_{W}+\left.N_{\mathcal{E}}\right|_{E_{n}}+\left.N_{\mathcal{E}}\right|_{E_{m}}} \\
& \rightarrow\left(z_{V} \otimes E_{n}\right)_{N_{V}+\left.N_{\mathcal{E}}\right|_{E_{n}}} \star\left(z_{W} \otimes E_{m}\right)_{N_{W}+\left.N_{\mathcal{E}}\right|_{E_{m}}} \simeq z_{I}^{\star} z_{W} .
\end{aligned}
$$

Corresponding to it there is a chiral pairing, defined for every $\mathcal{M}$ as above:

$$
\left.j_{x *} j_{x}^{*}\left(\left(\Gamma\left(\operatorname{Gr}_{G, X}, \mathcal{F}_{V \otimes W}\right) \otimes \mathcal{E}_{n} \otimes \mathcal{E}_{m}\right) \otimes \mathcal{M}\right)\right) \rightarrow \Delta_{!}\left(z_{V} \underset{I}{\star} z_{W} \underset{I}{\star} \mathcal{M}\right) .
$$


The third map in (57) equals the composition

$$
\begin{aligned}
& \left.j_{*} j^{*}\left(\left(\Gamma \operatorname{Gr}_{G, X}, \mathcal{F}_{V, X}\right) \otimes \mathcal{E}_{n}\right) \otimes\left(\Gamma\left(\operatorname{Gr}_{G, X}, \mathcal{F}_{W, X}\right) \otimes \mathcal{E}_{m}\right) \otimes \mathcal{M}\right) \rightarrow \\
& \rightarrow \Delta_{x_{1}=x_{2} !}\left(j_{x *} j_{x}^{*}\left(\left(\Gamma\left(\operatorname{Gr}_{G, X}, \mathcal{F}_{V \otimes W}\right) \otimes \mathcal{E}_{n} \otimes \mathcal{E}_{m}\right) \otimes \mathcal{M}\right)\right) \stackrel{(59)}{\longrightarrow} \Delta_{!}\left(z_{V} \underset{I}{\star} z_{W} \underset{I}{\star} \mathcal{M}\right) .
\end{aligned}
$$

Proposition 6.7. The sum of the three maps above is 0 .

Proof. Recall that

$$
\mathrm{Fl}_{G,(X-x)^{2}-\Delta_{X-x}} \simeq\left(\left(\operatorname{Gr}_{G, X-x} \times \operatorname{Gr}_{G, X-x}\right) \underset{(X-x)^{2}}{\times}\left((X-x)^{2}-\Delta_{X-x}\right)\right) \times \mathrm{Fl}_{G},
$$

and consider the twisted D-module

$$
j_{! *}\left(\left(\mathcal{F}_{V, X-x} \otimes \mathcal{E}_{n}\right) \otimes\left(\mathcal{F}_{W, X-x} \otimes \mathcal{E}_{m}\right) \otimes \delta_{1, \mathrm{Fl}_{G}}\right)
$$

on $\mathrm{Fl}_{G, X^{2}}$.

Let $\mathrm{Fl}_{G, X \times x}$ (resp., $\mathrm{Fl}_{G, x \times X}, \mathrm{Fl}_{G, \Delta_{X}}$ ) denote the preimage in $\mathrm{Fl}_{G, X^{2}}$ of the corresponding subvariety in $X^{2}$. By considering the iterated version of $\mathrm{Fl}_{G, X^{2}}$, the following description of the D-module (60) was obtained in [Ga1]:

- The restriction of the D-module (60) to $\mathrm{Fl}_{G, X \times x} \simeq \mathrm{Fl}_{G, X}$ identifies with

$$
\operatorname{ker}\left(j_{x *} j_{x}^{*}\left(\left(\mathcal{F}_{V, X} \otimes \mathcal{E}_{n}\right) \otimes z_{W}\right) \rightarrow i_{x !}\left(z_{V}{ }_{I} z_{W}\right)\right) .
$$

- The restriction of (60) to $\mathrm{Fl}_{G, x \times X} \simeq \mathrm{Fl}_{G, X}$ identifies with

$$
\operatorname{ker}\left(j_{x *} j_{x}^{*}\left(\left(\mathcal{F}_{W, X} \otimes \mathcal{E}_{m}\right) \otimes z_{V}\right) \rightarrow i_{x !}\left(\mathcal{Z}_{W}^{\star} z_{V}\right)\right) .
$$

- The restriction of (60) to $\mathrm{Fl}_{G, \Delta_{X}} \simeq \mathrm{Fl}_{G, X}$ identifies with

$$
\operatorname{ker}\left(j_{x *} j_{x}^{*}\left(\left(\mathcal{F}_{V \otimes W, X} \otimes \mathcal{E}_{n} \otimes \mathcal{E}_{m}\right) \otimes \delta_{1, \mathrm{Fl}_{G}}\right) \stackrel{(58)}{\longrightarrow} i_{x !}\left(z_{V} \underset{I}{z_{W}}\right)\right) \text {. }
$$

Hence, we obtain three maps

$$
j_{*} j^{*}\left(\left(\mathcal{F}_{V, X} \otimes \mathcal{E}_{n}\right) \otimes\left(\mathcal{F}_{W, X} \otimes \mathcal{E}_{m}\right) \otimes \delta_{1, \mathrm{Fl}_{G}}\right) \rightarrow \Delta_{!}\left(z_{V} \underset{I}{\star} z_{W}\right),
$$

whose some equals to zero. By lifting the terms of (61) and the corresponding maps by means of $\pi_{\mathrm{Fl}}: \mathrm{Jets}^{\mathrm{mer}}(G)_{X^{2} \times x} \rightarrow \mathrm{Fl}_{G, X^{2}}$, we obtain three maps

$j_{*} j^{*}\left(\left(\Gamma\left(\operatorname{Gr}_{G, X}, \mathcal{F}_{V, X}\right) \otimes \mathcal{E}_{n}\right) \otimes\left(\Gamma\left(\operatorname{Gr}_{G, X}, \mathcal{F}_{W, X}\right) \otimes \mathcal{E}_{m}\right) \otimes \delta_{I, G((t)))}\right) \rightarrow \Delta_{!}\left(z_{V \otimes W}^{\star} \delta_{I, G((t)))}\right)$.

We claim that these maps coincide with those of of Proposition 6.7 for $\mathcal{M}=$ $\delta_{I, G((t))}$. This follows from Lemma 2.12 in the same way as in the proof of Proposition 2.8 . 
The case of a general $\mathcal{M}$ follows from that of $\delta_{I, G((t))}$ by the construction of the maps (54).

6.8. Proof of Theorem 5.4. The proof will be parallel to that of Theorem 1.10. It suffices to construct the isomorphisms

$$
\mathfrak{s}_{V}^{-1}: \mathcal{V}_{\widetilde{\mathfrak{Z}} \mathfrak{g}, x}^{\text {int,nilp }} \underset{\widetilde{\mathfrak{Z}}_{\mathfrak{g}, x}^{\text {int,nilp }}}{\otimes} \mathcal{M} \simeq \mathcal{Z}_{V} \underset{I}{\star} \mathcal{M},
$$

for $\mathcal{M}=\delta_{I, G((t))}$, which commute with the right action of $\widehat{\mathfrak{g}}_{\text {crit }}$, which intertwine the actions of $N_{\mathcal{V}_{\tilde{\mathfrak{J}}_{\mathfrak{g}, x}^{\text {int,nilp }}}}$ and $N_{V}$, and which are compatible with tensor products of representations. Given $V \in \operatorname{Rep}(\check{G})$ and $\mathcal{M} \in \widehat{\mathfrak{g}}_{\text {crit }}$-mod ${ }^{I}$ will construct the

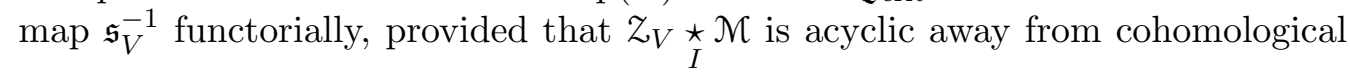
degree 0 for all $V \in \operatorname{Rep}(\check{G})$.

Let $\mathcal{N}_{1}$ and $\mathcal{N}_{2}$ be two $\widehat{\mathfrak{g}}_{\text {crit }}$-modules, such that the support of $\mathcal{N}_{1}$ over $\operatorname{Spec}\left(\mathfrak{Z}_{\mathfrak{g}}\right)$ is in $\operatorname{Spec}\left(\widetilde{\mathfrak{Z}}_{\mathfrak{g}, x}^{\text {int,nilp }}\right)$. The next assertion is deduced from Lemma 3.7 and Proposition 3.4 as was the case of Theorem 3.2:

Lemma 6.9. Chiral pairings

$$
\left\{\left(\mathcal{V}_{\mathfrak{z} \mathfrak{g}} \underset{\mathfrak{z g}}{\otimes} \mathcal{A}_{\mathfrak{g}, \text { crit }}\right) \underset{\mathcal{O}_{X}}{\otimes} \mathcal{E}_{n}, \mathcal{N}_{1}\right\} \rightarrow \mathcal{N}_{2}
$$

are in bijection with maps of $\widehat{\mathfrak{g}}_{\text {crit }}$-modules

$$
\left(\mathcal{V}_{\widetilde{\mathfrak{Z}}_{\mathfrak{g}, x}^{\text {int,nilp }}} \underset{\widetilde{\mathfrak{Z}}_{\mathfrak{g}, x}^{\text {int,nilp }}}{\otimes} \mathcal{N}_{1}\right)_{N_{\mathcal{\mathfrak { J }}_{\mathfrak{g}, x}^{\text {int,nilp }}}^{n}} \rightarrow \mathcal{N}_{2}
$$

More generally, for a local system $\mathcal{E}^{\prime}$ on $X-x$ with a nilpotent monodromy around $x$, chiral pairings

$$
\left\{\left(\mathcal{V}_{\mathfrak{z} \mathfrak{g}} \underset{\mathfrak{z} \mathfrak{g}}{\otimes} \mathcal{A}_{\mathfrak{g}, \text { crit }}\right) \underset{\mathcal{O}_{X}}{\otimes} \mathcal{E}^{\prime}, \mathcal{N}_{1}\right\} \rightarrow \mathcal{N}_{2}
$$

are in bijection with maps of $\widehat{\mathfrak{g}}_{\text {crit }}$-modules $\left(\left(\mathcal{V}_{\widetilde{\mathfrak{J}}_{\mathfrak{g}, x}^{\text {int,nilp }}} \underset{\widetilde{\mathfrak{J}}_{\mathfrak{g}, x}^{\text {int,nilp }}}{\otimes} \mathcal{N}_{1}\right) \otimes\right.$ $\left.\mathcal{E}_{x}^{\prime}\right)_{N_{\widetilde{\mathfrak{Z}}_{\mathfrak{g}, x} \text { int,nilp }}+N_{\mathcal{E}^{\prime}}} \rightarrow \mathcal{N}_{2}$

Setting $\mathcal{N}_{1}=\mathcal{M}$ and $\mathcal{N}_{2}=z_{V} \underset{I}{\star} \mathcal{M}$ and taking $n>>0$ so that $N_{\mathcal{V}_{\widetilde{\mathfrak{J}}_{\mathfrak{g}, x} \text { int,nilp }}^{n}}=0$, from the map (54), we produce the desired map

$$
\mathfrak{s}_{V}^{-1}: \mathcal{V}_{\widetilde{\mathfrak{Z}}_{\mathfrak{g}, x}^{\text {int,nilp }}} \underset{\widetilde{\mathfrak{Z}}_{\mathfrak{g}, x}^{\text {int,nilp }}}{\otimes} \mathcal{M} \rightarrow \mathcal{Z}_{V} \star \underset{I}{\mathcal{M}} .
$$


We claim that this map is compatible with tensor products of objects of $\operatorname{Rep}(\check{G})$, i.e., that the following three maps coincide:

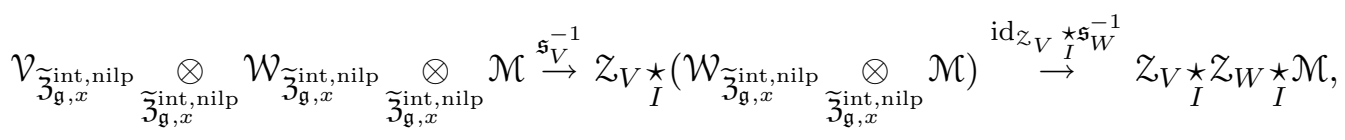

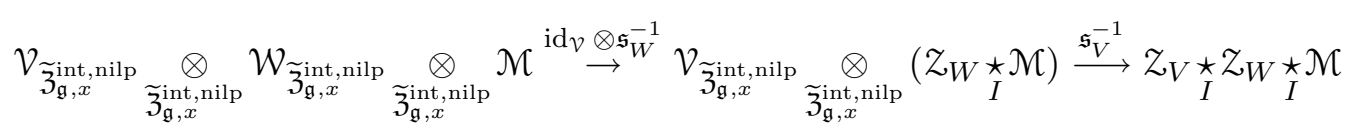

and

$$
\mathcal{V}_{\widetilde{\mathfrak{Z}}_{\mathfrak{g}, x}^{\text {int,nilp }}} \underset{\widetilde{\mathfrak{Z}}_{\mathfrak{g}, x}^{\text {int,nilp }}}{\otimes} \mathcal{W}_{\widetilde{\mathfrak{Z}}_{\mathfrak{g}, x}^{\text {int,nilp }}} \underset{\widetilde{\mathfrak{J}}_{\mathfrak{g}, x}^{\text {int,nilp }}}{\otimes} \mathcal{M} \stackrel{\mathfrak{s}_{V \otimes W}^{-1}}{\rightarrow} z_{V \otimes W} \underset{I}{\star \mathcal{M}} \simeq z_{V} \underset{I}{\star} z_{W} \underset{I}{\star} \mathcal{M} .
$$

This follows from Proposition 6.7 and Lemma 3.5 as in the proof of Proposition 3.9.

Finally, the fact that the maps $\mathfrak{s}_{V}^{-1}$ are isomorphisms follows from the above compatibility with tensor products as in the proof of Theorem 1.10.

Remark. Let us notice that the assertion of Theorem 6.6 is stronger than that of its spherical counterpart, Theorem 2.6. Indeed, the former theorem, combined with Lemma 6.9, implies the existence of an isomorphism (62) directly. In other words, the additional argument, involving duailization is not necessary.

\section{Convolution and twisting for general Chiral algebras, CONTINUED}

7.1. The goal of this section is to prove Theorem 6.6 and give another interpretation of the map (54), parallel to what was done in Sect. 4 in the spherical case.

We shall first discuss a generalization of the construction of Proposition 4.8. Let $\mathcal{A}$ be a chiral algebra as in Sect. 4.1 , and let $\left\{\mathcal{M}_{1}, \mathcal{M}_{2}\right\} \rightarrow \mathcal{M}_{3}$ be a chiral pairing between $\mathcal{A}$-modules.

Let also $\mathcal{F}_{1}^{\prime}, \mathcal{F}_{2}^{\prime}, \mathcal{F}_{3}^{\prime}$ be chiral modules over $\mathfrak{D}_{\kappa, G}$, and $\left\{\mathcal{F}_{1}^{\prime}, \mathfrak{F}_{2}^{\prime}\right\} \rightarrow \mathcal{F}_{3}^{\prime}$ be a chiral pairing.

Proposition-Construction 7.2. In the above situation there exists a naturally defined chiral pairing

$$
\left\{\mathcal{F}_{1}^{\prime} \widetilde{\nabla} \mathcal{M}_{1}, \mathcal{F}_{2}^{\prime} \widetilde{\nabla} \mathcal{M}_{2}\right\} \rightarrow \mathcal{F}_{3}^{\prime} \widetilde{\nabla} \mathcal{M}_{3},
$$

compatible with the actions of $\mathcal{A}$ and $\mathcal{A}_{\mathfrak{g}, 2 \kappa_{\text {crit }}}$. 
Proof. Each $\mathcal{F}_{i}^{\prime} \widetilde{\otimes} \mathcal{M}_{i}$ is isomorphic to $\mathcal{F}_{i}^{\prime} \otimes \mathcal{M}_{i}$ for $i=1,2,3$ as a D-module, and we define the desired pairing as the one coming from (41). The fact that this pairing is compatible with the action of chiral algebras follows from the construction of $\mathcal{F}_{i}^{\prime} \widetilde{\otimes} \mathcal{M}_{i}$ in Sect. 4.7 .

By multiplying the terms of the pairing of Proposition-Construction 7.2 by the chiral Clifford algebra we obtain a chiral pairing of complexes of $\mathcal{A}$-modules:

$$
\left\{\mathfrak{C}^{\frac{\infty}{2}}\left(L_{\mathfrak{g}}, \mathcal{F}_{1}^{\prime} \otimes \mathcal{M}_{1}\right), \mathfrak{C}^{\frac{\infty}{2}}\left(L_{\mathfrak{g}}, \mathfrak{F}_{2}^{\prime} \otimes \mathcal{M}_{2}\right)\right\} \rightarrow \mathfrak{C}^{\frac{\infty}{2}}\left(L_{\mathfrak{g}}, \mathfrak{F}_{3}^{\prime} \otimes \mathcal{M}_{3}\right) .
$$

Let us consider a particular case when $\mathcal{F}_{1}^{\prime} \simeq \mathfrak{D}_{G, \kappa}, \mathcal{F}_{2}^{\prime} \simeq \mathcal{F}_{3}^{\prime}=: \mathcal{F}^{\prime}$ and the pairing is given by the chiral action. Assume also that $\mathcal{M}_{1}$ is $\operatorname{Jets}(G)_{X}$-equivariant. In this case the map $\mathcal{M}_{1} \rightarrow \mathcal{O}_{\text {Jets }(G)_{X}} \otimes \mathcal{M}_{1}$ induces a map of complexes of $\mathcal{A}$-modules

$$
\mathcal{M}_{1} \rightarrow \mathfrak{C}^{\frac{\infty}{2}}\left(L_{\mathfrak{g}}, \mathcal{F}_{1}^{\prime} \otimes \mathcal{M}_{1}\right)
$$

Lemma 7.3. The resulting chiral pairing

$$
\left\{\mathcal{M}_{1}, \mathfrak{C}^{\frac{\infty}{2}}\left(L_{\mathfrak{g}}, \mathcal{F}^{\prime} \otimes \mathcal{M}_{2}\right)\right\} \rightarrow \mathfrak{C}^{\frac{\infty}{2}}\left(L_{\mathfrak{g}}, \mathcal{F}^{\prime} \otimes \mathcal{M}_{3}\right)
$$

coincides with that of (43).

7.4. Let us assume now that in the previous set-up the chiral $\mathfrak{D}_{G, \kappa}$-module $\mathcal{F}_{1}^{\prime}$ is $\operatorname{Jets}(G)_{X}$-equivariant on the right. Let $\mathcal{F}_{2}^{\prime}$ and $\mathcal{F}_{3}^{\prime}$ be both supported at $x \in X$, and assume that as twisted D-modules on $G((t))$ they are both $I$-equivariant on the right.

Let $\mathcal{M}_{1}$ be Jets $(G)_{X}$-equivariant, and let $\mathcal{M}_{2}, \mathcal{M}_{3}$ be supported at $x \in X$ and $I$-equivariant. In this case the pairing (63) gives rise to a chiral pairing

$$
\left\{\mathfrak{C}^{\frac{\infty}{2}}\left(L_{\mathfrak{g}} ; \mathfrak{g}, \mathcal{F}_{1}^{\prime} \otimes \mathcal{M}_{1}\right), \mathfrak{C}^{\frac{\infty}{2}}\left(L_{\mathfrak{g}} ; \mathfrak{h}, \mathcal{F}_{2}^{\prime} \otimes \mathcal{M}_{2}\right)\right\} \rightarrow \mathfrak{C}^{\frac{\infty}{2}}\left(L_{\mathfrak{g}} ; \mathfrak{h}, \mathcal{F}_{3}^{\prime} \otimes \mathcal{M}_{3}\right) .
$$

Let us specialize further the case when $\mathcal{M}_{1} \simeq \mathcal{A}, \mathcal{M}_{2} \simeq \mathcal{M}_{3}:=\mathcal{M}$, with the chiral pairing being given by the action. Let the terms of $\left\{\mathcal{F}_{1}^{\prime}, \mathcal{F}_{2}^{\prime}\right\} \rightarrow \mathcal{F}_{3}^{\prime}$ be those of (51) for $n>>0$. We obtain a chiral pairing

$$
\begin{aligned}
\left\{\mathfrak{C}^{\frac{\infty}{2}}\left(L_{\mathfrak{g}} ; \mathfrak{g}, \pi^{*}\left(\mathcal{F}_{V, X-x}\right) \otimes \mathcal{A}\right) \otimes \mathcal{E}_{n},\right. & \left.\mathfrak{C}^{\frac{\infty}{2}}\left(L_{\mathfrak{g}} ; \mathfrak{h}, \delta_{I, G((t))} \otimes \mathcal{M}\right)\right\} \rightarrow \\
& \rightarrow \mathfrak{C}^{\frac{\infty}{2}}\left(L_{\mathfrak{g}} ; \mathfrak{h}, \pi_{\mathrm{Fl}}^{*}\left(\mathcal{Z}_{V}\right) \otimes \mathcal{M}\right)
\end{aligned}
$$

Let us assume now that $\kappa$ is such that $\mathcal{F}_{V, X} \star \mathcal{A}$ is acyclic away from cohomological degree 0 . Passing to the 0 -th cohomology in the previous expression, we obtain a functorial chiral pairing

$$
\left.\left\{\left(\mathcal{F}_{V, X-x} \star \mathcal{A}\right) \otimes \mathcal{E}_{n}\right), \mathcal{M}\right\} \rightarrow h^{0}\left(\mathcal{Z}_{V} \underset{I}{\star} \mathcal{M}\right),
$$


such that the action of $N_{\mathcal{E}}$ on the LHS goes over to the action of $N_{V}$ on the RHS.

Parallel to the spherical case, we have the following generalization of Theorem 6.6:

\section{Theorem 7.5.}

(1) For any $\mathcal{M} \in \mathcal{A}$-mod ${ }_{x}^{I}$ the convolution $\boldsymbol{Z}_{V} \underset{I}{\star} \mathcal{M}$ is acyclic away from cohomological degree 0 .

(2) For all $n$ that are large enough, the covariant functor on $\mathcal{A}$-mod $\operatorname{mat}_{x}$ that sends an object $\mathcal{N}$ to the set of chiral pairings

$$
\left\{\left(\left(\mathcal{F}_{V, X-x} \star \mathcal{A}\right) \otimes \mathcal{E}_{n}\right), \mathcal{M}\right\} \rightarrow \mathcal{N}
$$

is representable by $\mathcal{z}_{V} \underset{I}{\star} \mathcal{M}$.

The proof of this theorem is based on the following construction. Let

$$
\left\{\left(\left(\mathcal{F}_{V, X-x} \star \mathcal{A}\right) \otimes \mathcal{E}_{n}\right), \mathcal{M}\right\} \rightarrow \mathcal{N}^{\bullet}
$$

be a chiral pairing of complexes of $\mathcal{A}$-modules, where $\mathcal{N}^{\bullet}$ is a strongly $\operatorname{Jets}(G)_{X^{-}}$ equivariant complex.

Then for $m>>0$, from (64) we obtain a chiral pairing of complexes $\left\{\mathfrak{C}^{\frac{\infty}{2}}\left(L_{\mathfrak{g}} ; \mathfrak{g},\left(\pi^{*}\left(\mathcal{F}_{V^{*}, X}\right) \otimes \mathcal{E}_{m}\right) \otimes\left(\left(\mathcal{F}_{V, X} \star \mathcal{A}\right) \otimes \mathcal{E}_{n}\right)\right), \mathcal{M}\right\} \rightarrow \mathfrak{C}^{\frac{\infty}{2}}\left(L_{\mathfrak{g}} ; \mathfrak{h}, \pi_{\mathrm{Fl}}^{*}\left(\mathcal{Z}_{V^{*}}\right) \otimes \mathcal{N}^{\bullet}\right)$, and by passing to the 0 -th cohomology and composing with $\delta_{1, \operatorname{Gr}_{G, X}} \rightarrow \mathcal{F}_{V, X} \star$ $\mathcal{F}_{V^{*}, X}$ and $\mathcal{E}_{m+n} \rightarrow \mathcal{E}_{m} \otimes \mathcal{E}_{n}$, we obtain a chiral pairing

$$
\left\{\left(\mathcal{A} \otimes \mathcal{E}_{m+n}\right), \mathcal{M}\right\} \rightarrow h^{0}\left(z_{V^{*} \underset{I}{\star}} \mathcal{N}^{\bullet}\right) .
$$

Any such pairing factors through $\mathcal{E}_{m+n} \rightarrow \mathcal{O}_{X}$, and therefore corresponds to a map $\mathcal{M} \rightarrow h^{0}\left(\mathcal{F}_{V^{*}} \underset{I}{\star} \mathcal{N}^{\bullet}\right)$. The rest of the argument repeats that of the the proof of Theorem 4.14 .

\section{Appendix: proof of Proposition 2.12}

8.1. Let $\mathcal{F}_{X}$ be a $\kappa$-twisted D-module on $\operatorname{Gr}_{G, X}$ and let $\mathcal{F}_{X}^{\prime}$ denote its pull-back to Jets ${ }^{\mathrm{mer}}(G)_{X}$. Consider the D-module $\left(\mathbf{1}_{1,1}\right) !\left(\omega_{X} \otimes \mathcal{F}_{X}\right)$ on $\mathrm{Gr}_{G, X^{2}}$. Corresponding to it there is a map

$$
j_{*} j^{*}\left(\delta_{1, \operatorname{Gr}_{G, X}} \otimes \mathcal{F}_{X}\right) \rightarrow \Delta_{!}\left(\mathcal{F}_{X}\right)
$$

of D-modules on $\mathrm{Gr}_{G, X^{2}}$. Lifting this map by means of $\pi$ we obtain a map

$$
j_{*} j^{*}\left(\mathfrak{D}_{G, \kappa} \otimes \Gamma\left(\operatorname{Jets}^{\operatorname{mer}}(G)_{X}, \mathcal{F}_{X}^{\prime}\right)\right) \rightarrow \Delta_{!}\left(\Gamma\left(\operatorname{Jets}^{\operatorname{mer}}(G)_{X}, \mathcal{F}_{X}^{\prime}\right)\right) .
$$


Along with Proposition 2.12 we will prove the following:

Proposition 8.2. The map of (66) equals the map corresponding to the chiral action of $\mathfrak{D}_{G, \kappa}$ on $\Gamma\left(\operatorname{Jets}^{\mathrm{mer}}(G)_{X}, \mathcal{F}_{X}^{\prime}\right)$.

Applying this to $\mathcal{F}_{X}=\delta_{1, \mathrm{Gr}_{G, X}}$, we obtain a description of the chiral bracket on $\mathfrak{D}_{G, \kappa}$ in terms of distributions on $\operatorname{Jets}^{\operatorname{mer}}(G)_{X^{2}}$.

8.3. Since the chiral algebra $\mathfrak{D}_{G, \kappa}$ is generated by $\mathcal{O}_{\operatorname{Jets}(G)_{X}}$ and $L_{\mathfrak{g}, \kappa}$, to prove both Proposition 2.12 and Proposition 8.2, it suffices to show that the two maps in question coincide when instead of $\mathfrak{D}_{G, \kappa}$, as one of the multiples in the LHS, we take $\mathcal{O}_{\operatorname{Jets}(G)_{X}}$ or $L_{\mathfrak{g}, \kappa}$.

The assertion concerning $\mathcal{O}_{\operatorname{Jets}(G)_{X}}$ follows tautologically from the definition of the group ind-scheme $\operatorname{Jets}^{\text {mer }}(G)_{X^{2}}$. In the case of $L_{\mathfrak{g}, \kappa}$ we shall discuss the set-up of Proposition 2.12, while the case of Proposition 8.2 is similar. We need to establish the following:

Let $\mathbf{g}$ be any point of $G((t)) / K$ and let $\mathbf{1}_{1,1}(\mathrm{~g})$ be the corresponding section $X \rightarrow \mathrm{Gr}_{G, X ; K}$. The normal to this section, considered as a right D-module on $X-x$, has the property that

$$
\left.\left.\mathcal{N}_{\mathbf{1}_{1,1}(\mathbf{g})}\right|_{X-x} \simeq L_{\mathfrak{g}}\right|_{X-x} \oplus T_{\mathbf{g}}(G((t)) / K) \otimes \omega_{X-x} \text { and }\left.\mathcal{N}_{\mathbf{1}_{1,1}(\mathbf{g})}\right|_{x} \simeq T_{\mathbf{g}}(G((t)) / K) .
$$

In particular, we obtain a map

$$
j_{x *} j_{x}^{*}\left(L_{\mathfrak{g}}\right) \rightarrow i_{x !}\left(T_{\mathbf{g}}(G((t)) / K)\right),
$$

which is equivalent to a map

$$
\mathfrak{g}((t)) \simeq H_{D R}^{0}\left(\mathcal{D}_{x}^{\times}, L_{\mathfrak{g}}\right) \rightarrow T_{\mathbf{g}}(G((t)) / K) .
$$

We need to show that the latter map equals the natural projection

$$
\mathfrak{g}((t)) \rightarrow \mathfrak{g}((t)) / T_{\mathbf{g}}(G((t)) / K),
$$

corresponding to the left action of $G((t))$ on $G((t)) / K$. Using the above $G((t))$ action, we reduce the assertion to the case when $\mathbf{g}$ is the unit point of $G((t))$, in which case $T_{\mathbf{g}}(G((t)) / K) \simeq \mathfrak{g}((t)) / \mathrm{k}$, where $\mathrm{k}$ is the Lie algebra of $K$.

Thus, we need to show that the map $\left.\left.L_{\mathfrak{g}}\right|_{X-x} \rightarrow \mathcal{N}_{\mathbf{1}_{1,1}(\mathbf{g})}\right|_{X-x}$ extends to a map of D-modules $L_{\mathfrak{g} . \mathrm{k}} \rightarrow \mathcal{N}_{\mathbf{1}_{1,1}(\mathrm{~g})}$, where

$$
L_{\mathfrak{g} . \mathrm{k}}:=\operatorname{ker}\left(j_{x *} j_{x}^{*}\left(L_{\mathfrak{g}}\right) \rightarrow i_{x !}(\mathfrak{g}((t)) / \mathrm{k})\right) .
$$

This is a direct calculation performed below. 
8.4. Without restriction of generality, we can assume that $X$ is affine, and let $\xi\left(x_{1}, x_{2}\right)$ be a $\mathfrak{g}$-valued map on $X \times X-\left(\Delta_{X} \sqcup X \times x\right)$. We will show that $\xi\left(x_{1}, x_{2}\right)$ gives rise to a vertical vector field on $\mathrm{Gr}_{G, X ; K}$, relative to its projection onto $X$, and that the arising normal vector field to the unit section vanishes if and only if $\xi\left(x_{1}, x_{2}\right)$ has no poles at the diagonal, and for any fixed $x_{1}$ the Laurent expansion of $\xi\left(x_{1}, \cdot\right)$ at $x_{2}=x$, viewed as an element of $\mathfrak{g}((t))$, belongs to $\mathrm{k}$. This will imply the required assertion.

It will be more convenient to use a group-theoretic notation. I.e., we will think of $\xi\left(x_{1}, x_{2}\right)$ as a map $X \times X-\left(\Delta_{X} \sqcup X \times x\right) \rightarrow G$, parameterized by the scheme of dual numbers. More generally, we will work with a map $\mathbf{g}\left(x_{1}, x_{2}\right)$ : $\left(X \times X-\left(\Delta_{X} \sqcup X \times x\right)\right) \times S \rightarrow G$ for an arbitrary scheme $S$.

To $\mathbf{g}\left(x_{1}, x_{2}\right)$ as above we attach an $S$-valued automorphism of $\operatorname{Gr}_{G, X ; K}$ as follows. Given a point $\left(x_{1}, \mathcal{P}_{G}, \beta, \alpha\right)$ of $\mathrm{Gr}_{G, X ; K}$ we define a new point by leaving $\left(x_{1}, \mathcal{P}_{G}, \alpha\right)$ the same, but multiplying $\beta$ by $\mathbf{g}\left(x_{1}, \cdot\right)$, thought of as a $G$-valued function on $X-\left(x_{1} \sqcup x\right)$.

Applying this automorphism to the unit section of $\mathrm{Gr}_{G, X ; K}$, the resulting new $S \times X$-valued point of $\operatorname{Gr}_{G, X ; K}$ will be isomorphic to the initial one if and only if there exists an $S \times X$-valued automorphism of $\mathcal{P}_{G}^{0}$, preserving $\alpha$, and whose value at any $x_{1} \in X$ equals that of $\mathbf{g}\left(x_{1}, \cdot\right)$. But this precisely means that $\mathbf{g}\left(x_{1}, x_{2}\right)$ extends regularly to the diagonal and $X \times x$, and the Taylor expansion of $\mathbf{g}\left(x_{1}, \cdot\right)$ around $x$ belongs to $K$.

\section{REFERENCES}

[AG] S. Arkhipov and D. Gaitsgory, Differential operators on the loop group via chiral algebras, Int. Math. Res. Not. 2002, no. 4, 165-210.

[BL] A. Beauville, Y. Laszlo, Un lemme de descente, C.R. Acad. Sci. Paris, Sér. I Math. 320 (1995) 335-340.

[Be] A. Beilinson, How to glue perverse sheaves, in: $K$-theory, arithmetic and geometry (Moscow, 1984-1986), 42-51, Lecture Notes in Math., 1289, Springer, Berlin, 1987.

[BB] A. Beilinson and J. Bernstein, A proof of Jantzen conjectures, Advances in Soviet Mathematics 16, Part 1, pp. 1-50, AMS, 1993.

[CHA] A. Beilinson and V. Drinfeld, Chiral algebras, American Mathematical Society Colloquium Publications 51, AMS, 2004.

[BD] A. Beilinson and V. Drinfeld, Quantization of Hitchin's integrable system and Hecke eigensheaves, available at http://www.math.uchicago.edu/ arinkin/langlands/

[FF] B. Feigin and E. Frenkel, Affine Kac-Moody algebras at the critical level and Gelfand-Dikii algebras, in Infinite Analysis, eds. A. Tsuchiya, T. Eguchi, M. Jimbo, Adv. Ser. in Math. Phys. 16, 197-215, Singapore: World Scientific, 1992.

[F] E. Frenkel, Wakimoto modules, opers and the center at the critical level, Adv. Math. 195 (2005) 297-404.

[FB] E. Frenkel and D. Ben-Zvi, Vertex Algebras and Algebraic Curves, Mathematical Surveys and Monographs 88, Second Edition, AMS, 2004. 
[FG1] E. Frenkel and D. Gaitsgory, D-modules on the affine Grassmannian and representations of affine Kac-Moody algebras, Duke Math. J. 125 (2004) 279-327.

[FG2] E. Frenkel and D. Gaitsgory, Local geometric Langlands correspondence and affine KacMoody algebras, Preprint math.RT/0508382.

[FHL] I. Frenkel, Y.-Z. Huang and J. Lepowsky, On axiomatic approaches to vertex operator algebras and modules. Mem. Amer. Math. Soc. 104 (1993), no. 494.

[Ga] D. Gaitsgory, Construction of central elements in the Iwahori Hecke algebra via nearby cycles, Invent. Math. 144 (2001), 253-280.

[Ga1] D. Gaitsgory, Braiding compatibilities, an appendix to a paper by R. Bezrukavnikov and V. Ostrik, in: Representation theory of algebraic groups and quantum groups, 91-100, Adv. Stud. Pure Math. 40, Math. Soc. Japan, Tokyo (2004).

[MV] I. Mirković and K. Vilonen, Geometric Langlands duality and representations of algebraic groups over commutative rings, Preprint math.RT/0401222.

Edward Frenkel

Department of Mathematics, University of California

Berkeley, CA 94720, USA

E-mail: frenkel@math.berkeley.edu

Dennis Gaitsgory

Department of Mathematics, Harvard University

Cambridge, MA 02138, USA

E-mail: gaitsgory@math.harvard.edu 